\title{
Scanning Tunneling Microscopy Studies of Organic Monolayers Adsorbed on the Rhodium(111) Crystal Surface
}

\author{
Paul Davis Cernota \\ Ph.D. Thesis \\ Department of Chemistry \\ University of California \\ and \\ Materials Sciences Division \\ Lawrence Berkeley National Laboratory \\ University of California \\ Berkeley, California 94720
}

August 1999 


\section{DISCLAIMER}

This report was prepared as an account of work sponsored by an agency of the United States Government. Neither the United States Government nor any agency thereof, nor any of their employees, make any warranty, express or implied, or assumes any legal liability or responsibility for the accuracy, completeness, or usefulness of any information, apparatus, product, or process disclosed, or represents that its use would not infringe privately owned rights. Reference herein to any specific commercial product, process, or service by trade name, trademark, manufacturer, or otherwise does not necessarily constitute or imply its endorsement, recommendation, or favoring by the United States Government or any agency thereof. The views and opinions of authors expressed herein do not necessarily state or reflect those of the United States Government or any agency thereof. 


\section{DISCLAIMER}

Portions of this document may be illegible in electronic image products. Images are produced from the best available original document. 
Scanning Tunneling Microscopy Studies of Organic Monolayers

Adsorbed on the Rhodium(111) Crystal Surface

by

Paul Davis Cernota

A.B. (Princeton University) 1995

A dissertation submitted in partial satisfaction of the

Requirements for the degree of

Doctor of Philosophy

in

Chemistry

in the

GRADUATE DIVISION

of the

UNIVERSITY OF CALIFORNIA, BERKELEY

Committee in charge:

Professor Gabor Somorjai, chair

Professor A. Paul Alivisatos

Professor Norman E. Phillips

Professor Roya Maboudian

Fall 1999 
Scanning Tunneling Microscopy Studies of Organic Monolayers Adsorbed on the Rhodium(111) Crystal Surface

Copyright 1999

by

Paul Davis Cernota 


\author{
Abstract \\ Scanning Tunneling Microscopy Studies of Organic Monolayers \\ Adsorbed on the Rhodium(111) Crystal Surface \\ By \\ Paul Davis Cernota \\ Doctor of Philosophy in Chemistry \\ University of California, Berkeley \\ Professor Gabor Somorjai, Chair
}

Scanning Tunneling Microscopy studies were carried out on ordered overlayers on the (111) surface of rhodium. These adsorbates include carbon monoxide (CO), cyclohexane, cyclohexene, 1,4-cyclohexadiene, para-xylene, and meta-xylene.

Coadsorbate systems included: $\mathrm{CO}$ with ethylidyne, $\mathrm{CO}$ with para- and meta-xylene, and para- xylene with meta-xylene.

In the case of $\mathrm{CO}$, the structure of the low coverage $(2 \times 2)$ overlayer has been observed. The symmetry of the unit cell in this layer suggests that the $\mathrm{CO}$ is adsorbed in the 3-fold hollow sites. There were also two higher coverage surface structures with $(\sqrt{ } 7 \mathrm{x} \sqrt{7})$ unit cells. One of these is composed of trimers of $\mathrm{CO}$ and has three $\mathrm{CO}$ molecules in each unit cell. The other structure has an additional $\mathrm{CO}$ molecule, making a total of four. This extra CO sits on a top site. 
When $\mathrm{CO}$ is added to a disordered ethylidyne covered surface, an ordered overlayer forms with $\mathrm{c}(4 \times 2)$ symmetry. Ordered domains were observed, sometimes two different domains in the same image. The ordered domains only show one type of feature, not two as would be expected. This feature has a larger corrugation than has been observed for $\mathrm{CO}$ on the surface. Due to the difference in heights of the molecules when they are adsorbed on the surface, as well as energetic considerations, it is most likely that the imaged molecule is ethylidyne, not $\mathrm{CO}$.

Three cyclic molecules were adsorbed on the surface, cyclohexane, cyclohexene and 1,4-cyclohexadiene. No ordered domains were observed for cyclohexane. Cyclohexene adsorbed on the surface in two different ordered structures, both of which correspond to the structures formed by the coadsorption of benzene and $\mathrm{CO}$. The molecules in both of these domains, the $(3 \times 3)$ and the $c(2 \sqrt{3} \times 4)$ structures, had high symmetry. It is most likely that the cyclohexene has dehydrogenated to form benzene. When 1,4-cyclohexadiene is adsorbed on the surface, it also forms the $c(2 \sqrt{3} \times 4)$ structure, indicating the conversion to benzene. When the surface is exposed to hydrogen gas before the adsorption of 1,4-cyclohexadiene, then two different ordered domains are observed on the surface. One domain is likely the $c(2 \sqrt{3} \times 4)$ structure. The other domain structure may consist of unconverted 1,4-cyclohexadiene caused by the blockage of the $\mathrm{H}$ atom adsorption sites by the exposure to hydrogen gas.

Two aromatic molecules of the benzene family, para- and meta-xylene, were imaged with STM on Rh(111) at room temperature. The molecular images have peculiar shapes that reflect their atomic and orbital structures. These shapes are diamonds and triangles with two bright corners that have been assigned to the methyl groups. Para- 
xylene forms ordered domains with three orientations, with one mirror plane of the molecule aligned parallel to the [11 2$]$-type directions. Meta-xylene molecules do not form ordered domains at room temperature, although individual molecules have a welldefined angular orientation relative to the substrate. In the case of low coverage, only at the step edges is the mobility low enough for stable imaging.

Coadsorption with $\mathrm{CO}$ was investigated in the case of meta-xylene. $\mathrm{CO}$ prevented diffusion of the molecules and formed a (2x2) structure surrounding the isolated xylene molecules. We conclude that the center of the triangular meta-xylene image is located in either the bridge or 3-fold hollow sites if the $\mathrm{CO}$ molecule adsorbs on top sites, and on top or 3-fold hollow sites if the $\mathrm{CO}$ molecule adsorbs on a 3-fold hollow site. Coadsorption of para-xylene and meta-xylene together showed a mixed layer of molecules rather than separate domains. The molecules in these domains could be identified as para- or meta-xylene by the their distinct shapes. 


\section{Table of Contents}

Page

List of Figures

Acknowledgements

Chapter 1. Introduction 1

Chapter 2. Surface Science 5

Introduction

Kinetic Theory of Gasses

Single Crystal Metal Surfaces

Chapter 3. Experimental Apparatus

Introduction

Sample Preparation

Auger Electron Spectroscopy

Low Energy Electron Diffraction

Chapter 4. Scanning Tunneling Microscopy

Introduction

Theory of Operation

Instrument Design

Chapter 5. Carbon Monoxide

Introduction

Experimental

Low Coverage $(2 \times 2)$ Structure

The (2x1) Structure 
Chapter 5. (cont.)

High Coverage Structures

Discussion

Chapter 6. Ethylidyne

Introduction

Experimental

Results

Discussion

Chapter 7. Cyclohexane Derivatives

68

Introduction

Experimental

Cyclohexane Adsorption

Cyclohexene Adsorption

1,4 Cyclohexadiene Adsorption

Discussion

Chapter 8. Para- and Meta-Xylene

Introduction

Experimental

Para-Xylene

Meta-Xylene

Meta-and Para-Xylene Coadsorption

Discussion

Chapter 9. Conclusion 


\section{List of Figures}

Figure 2-1: The structure of Rhodium metal.

Figure 2-2: The high-symmetry surface structures.

Figure 2-3: Five examples of ordered overlayers.

Figure 3-1: A schematic diagram of the UHV chamber.

Figure 3-2: A photograph of the sample holder.

Figure 3-3: A diagram of the Auger Process.

Figure 3-4: A diagram of the Auger Electronics.

Figure 3-5: A sample Auger spectrum of the rhodium surface.

Figure 3-6: A drawing of the LEED system components.

Figure 3-7: Correspondence of the LEED pattern with surface order.

Figure 4-1: A diagram of the metal-insulator-metal tunneling process.

Figure 4-2: An illustration of constant height and constant current modes.

Figure 4-3: An illustration of the STM.

Figure 5-1: Topographic image of the low coverage $(2 \times 2)-\mathrm{CO}$ structure.

Figure 5-2: Symmetry of the (2x2)-CO unit cell.

Figure 5-3: Topographic image of the (2x1) .5ML structure.

Figure 5-4: Topographic image of the $(\sqrt{ } 7 x \sqrt{ } 7)$ low coverage structure.

Figure 5-5: A comparison of the two $(\sqrt{7} \mathrm{x} \sqrt{7})$ structures formed by $\mathrm{CO}$.

Figure 6-1: Drawings of the (2x2)- $\mathrm{C}_{2} \mathrm{H}_{3}$ and $\mathrm{c}(4 \times 2)-\mathrm{C}_{2} \mathrm{H}_{3}+\mathrm{CO}$ structures.

Figure 6-2: LEED patterns of the $(1 \times 1)$ clean surface and the $c(4 \times 2)-\mathrm{C}_{2} \mathrm{H}_{3}+\mathrm{CO}$ structure.

Figure 6-3: Topographic image of the $c(4 \times 2)-\mathrm{C}_{2} \mathrm{H}_{3}+\mathrm{CO}$ structure. 
Figure 6-4: Corrugation of the ordered $c(4 \times 2)-\mathrm{C}_{2} \mathrm{H}_{3}+\mathrm{CO}$ overlayer.

Figure 6-5: An STM image and a schematic drawing of two domains of c(4x2)-

$$
\mathrm{C}_{2} \mathrm{H}_{3}+\mathrm{CO} \text {. }
$$

Figure 6-6: A diagram of the adsorption structure based on LEED data.

Figure 7-1: The structure of the $\mathrm{C}_{6}$ cyclic molecules.

Figure 7-2: Atomically resolved rhodium used for distance calibration.

Figure 7-3: Topographic image of the low-exposure cyclohexene overlayer.

Figure 7-4: Topographic image of the cyclohexene $(3 \times 3)$ structure.

Figure 7-5: Topographic image of a second cyclohexene structure.

Figure 7-6: An image showing the size of the $c(2 \sqrt{ } 3 \times 4)$ unit cell.

Figure 7-7: A low-bias-voltage image showing the ringed nature of the adsorbed molecules.

Figure 7-8: A topographic image of the 1,4-cyclohexadiene adsorption structure.

Figure 7-9: An STM image of the surface after pre-adsorption of $\mathrm{H}_{2}$ and exposure to 1,4-cyclohexadiene.

Figure 7-10: An image showing the size of the larger unit cell.

Figure 7-11: A figure showing the size of the smaller unit cell.

Figure 7-12: The corrugations of the molecules in the two ordered domains.

Figure 8-1: A diagram of the para- and meta-xylene molecules.

Figure 8-2: Topographic images of the adsorbed layer of para-xylene.

Figure 8-3: An STM image showing the orientation of the para-xylene molecules.

Figure 8-4: A topographic image of the low-coverage para-xylene overlayer.

Figure 8-5: Corrugations of the para-xylene molecules. 
Figure 8-6: A topographic image of meta-xylene adsorbed on the Rh(111) surface.

Figure 8-7: Topographic images showing the movement of meta-xylene in the ordered domains.

Figure 8-8: Topographic images showing the orientation of the meta-xylene molecules on the surface.

Figure 8-9: Close-up STM images of meta-xylene.

Figure 8-10: Location of the adsorbed meta-xylene molecules.

Figure 8-11: A topographic image of the coadsorption of para- and meta-xylene. 


\section{Acknowledgements}

While a Ph.D. dissertation may be written by a single individual, its production is not possible without a large number of people. First, special thanks are due to my research advisor, Professor Gabor Somorjai for allowing me to work in his research group and for the support, both scientific and fiscal, which he has given me during the last four years. It is special to have the opportunity to work with a scientist with such great vision and focus. Also, the guidance of Dr. Miquel Salmeron in both the Science of STM and LEED has been of great help. I'm convinced that in addition to his great ability to interpret data, he can troubleshoot any experimental problem that could possibly arise.

Of course, a graduate student is part of a research group, and the group part of that phase is essential to making it through graduate school with your sanity intact. In particular, I'd like to thank Alex Yoon, who taught me just about everything I know about a UHV vacuum system and introduced me to the Scanning Tunneling Microsecope. Also, John Jenson, Kieth Rider, Marc Rose were very helpful when I needed advice on my microscope and STM materials. Thanks to all of the members of the Somorjai and Salmeron groups - if I named you all, I'd have to go on for many pages - but particularly Joel, Enrico, David, Craig(s), Pete, Kyle, Aaron, Saskia, Keith(s), Yong, and Seong who let me enjoy my time at lab and in Berkeley. And, we all know that nothing gets accomplished in a research group without the help of the administrative assistants, Bob, Mae, Noreen, and Denise, who made sure my life wasn't spent filling out forms or drowning in paperwork. The technical staff of the college and the lab have been great 
when things needed procurement or fixing, particularly Henry in the electronics shop and James Wu for providing metals and wires for use in the STM.

Outside of the lab, thanks go to my roommates, past and persent: Boyd, Dave, Joel, and Hans. Having a great place to come home to after so much time at lab makes life so much easier. Too many nights were spent slogging through problem sets with Boyd, Dave(s), Fred, Kathy, and others, thanks for the friendship and the hard work. Thanks to Karen, Sharon Beth, and Jonathan for reminding me that life exists outside of Berkeley. Family holidays would have been dreadful without the great hospitality of my uncle Ray and aunt Kay as well as Julie, Thadd, Brandon, Timmy, Helen, Jim, Gunner and Sierra. Thanks for letting me be a part of the west coast Cernotas.

For my parents, who have twice lost the battle to keep me in Boston, I want to give special thanks for letting me go where I needed to be. Their invaluable and constant support from across the country has made graduate school a much better place. Don't worry, I'll be in New England next year. 


\section{Chapter 1: Introduction}

The physical properties of solid surfaces play an important role in many chemical processes. Surface chemistry was instrumental in the study of heterogeneous catalysis dating back to the early 1800 's, and the applications of the subfield of colloid chemistry was crucial to the paint and rubber industries in the early $20^{\text {th }}$ century [1]. These studies focused on the macroscopic properties of the surface. More recently, however, the atomic structure of the surface and the molecular-level interaction at the surfaces has been the focus of much scientific research. With the advent of Auger Spectroscopy as a tool for determining the cleanliness of a metal surface in 1968 [2], and the development of modern Low Energy Electron Diffraction electronics in the early 1960's[3,4], scientists realized the ability to prepare well defined clean metal surfaces for experiments. The study of surfaces at the atomic level was further enhanced by the development of the Scanning Tunneling Microscope (STM) by Binning and Rohrer [5] which was the first instrument capable of giving a topographic image of the surface without the assistance of theoretical modeling. 
This work focuses on the application of Scanning Tunneling Microscopy to a number of systems important for their application in catalysis and for the advancement of the theory of the operation of a Scanning Tunneling Microscope.

The metal used in these experiments, rhodium, has important applications in the conversion of $\mathrm{CO}$ to $\mathrm{CO}_{2}$ and $\mathrm{NO}$ to $\mathrm{N}_{2}$ in the catalytic converters in cars, for example. In addition, rhodium bonds strongly with surface adsorbates, so that the diffusion of molecules on the surface at room temperature is slow compared to the time it takes to record an STM image. This is an important consideration given the high diffusion rates of molecules on other important transition metal surfaces such as platinum [6]. The (111) surface was chosen because it is the most thermodynamically stable, and therefore most likely to be present in a faceted foil or small metal particles.

In order to study the interactions of molecules with the surface, it is important to look at the first layer that adsorbs on the surface, since this layer is affected most by the surface-adsorbate coupling. This is accomplished by adsorbing a single monolayer of a substance on a surface, and using the STM to image the adsorbed monolayer. Since in liquids and gasses, the intermolecular interactions are much weaker than the surfacemolecule interactions, this can be accomplished with a wide variety of chemicals. Multiple layers of adsorbates are likely to desorb since the intermolecular interaction are weak. The strong surface-adsorbate interaction in the first layer means that this layer is more likely to remain on the metal surface.

One system of importance is the adsorbed layer of Carbon Monoxide (CO). Since rhodium is an important catalyst for the elimination of $\mathrm{CO}$ from combustion exhaust, this is a natural choice. In addition, past studies of systems with $\mathrm{CO}$ adsorbed on the surface 
with other chemicals $[7,8]$ has shown that $\mathrm{CO}$ has the ability to induce the ordering of molecules on the surface which would not be ordered if adsorbed alone on the surface. In order to look at these coadsorption systems in detail, an understanding of the properties of $\mathrm{CO}$ on the rhodium surface is required.

Other systems which are studied in this work are the coadsorption of $\mathrm{CO}$ and ethylene, $\mathrm{C}_{2} \mathrm{H}_{4}$ (which converts to ethylidyne, $\mathrm{C}_{2} \mathrm{H}_{3}$ on the $\mathrm{Rh}(111)$ surface), the adsoption of cyclohexane, and the adsorption of para-xylene and meta-xylene. The coadsorption system is important to study the ordering effect of $\mathrm{CO}$. Cyclohexane and molecules which result from its dehydrogenation, such as cyclohexene and benzene, is a first step towards studying a molecular reaction which may be induced on the surface at very localized location. The study of xylenes gives an insight as to how the spacial positions of small functional groups (in this case, $\mathrm{CH}_{3}$ ) affect the shape of the molecule in the STM image.

In this dissertation, there is first an introduction to the language and basic concepts of surface science, and a description of the experimental apparatus used. Then there is a description of the theory and operation of the Scanning Tunneling Microscope. The experiments are described in four chapters, in the order: $\mathrm{CO}, \mathrm{CO}$ plus ethylidyne, cyclohexane derivatives and para- and meta-xylene. 
References

[1] G. A. Somorjai, Introduction to Surface Chemistry and Catalysis, John Wiley and Sons, New York, NY, 1994.

[2] G. Ertl and J. Küppers, Low Energy Electrons and Surface Chemistry, Weinheim: Verlag Chimie; 1974.

[3] E.J. Scheibner, L.H. Germer, and C.D. Hartmann, Rev. Sci. Instr. 31 (1960) 112.

[4] J.J. Lander, F. Unterwald, and J Morrison, Rev. Sci. Instr. 33 (1962) 784.

[5] Binning, Rohrer, Gerber, and Weibel, Phys. Rev. Lett. 49 (1982) 57.

[6] H. A. Yoon, Ph.D. Thesis, University of California, Berkeley, 1996.

[7] A. Gavezzotti, M. Simonetta, M.A. Van Hove, and G.A. Somorjai, Surf. Sci. 154 (1985) 109.

[8] C. Mate and G.A. Somorjai, Surf. Sci. 160 (1985) 542. 


\section{Chapter 2: Surface Science}

\section{Introduction}

Understanding the surface properties of materials is an important part of understanding many chemical systems. Historically important disciplines such as catalysis have the chemistry of surfaces as fundamental aspects of their mechanisms. In addition, the knowledge of surface properties has become increasingly important with the advent of the miniaturization of many electronic components. One way to investigate the surface properties of materials is to design model systems which reduce the number of extraneous interactions. Model systems in surface science generally include two components, a single crystal surface and an Ultra High Vacuum (UHV) system. Single crystal surfaces can be well-characterized so that the relationship between chemical processes and the morphology of the surface can be investigated. UHV systems allow the cleaning of the samples and provide an atmosphere where the surface can remain atomically clean for an extended time. This provides the ability to keep a surface wellcharacterized throughout the course of an experiment. Here we discuss single crystal 
surfaces, the kinetic theory of gasses (which governs the properties of low pressure systems like UHV chambers) and other basic concepts in surface science.

\section{Kinetic Theory of Gasses}

In order to study well characterized surfaces, it is important to be able to keep the surface clean of contaminants. For a metal surface, the two main contaminants will be impurity atoms from the bulk of the metal and the adsporption of gasseous molecules. The impurities from the bulk diffuse to the surface at a very low rate in the normal temperature range of most experiments. Therefore, the majority of the contaminants will come from the gas phase. These adsorbants can be reduced by operating at very low pressures. The magnitude of the pressure can be calculated from the kinetic theory of gasses. This theory allows us to calculate the number of molecules which impinge on the surface in a given time at a given temperature and pressure. The equation for the flux of molecules striking a unit area of the surface is given by [3],

$$
\text { Flux }\left(\frac{\text { atoms }}{\mathrm{cm}^{2} \cdot \sec }\right)=\frac{N_{A} \cdot P(P a)}{\sqrt{2 \pi M(g / m o l e) R T}}
$$

which is equivalent to:

$$
\text { Flux }\left(\frac{\text { atoms }}{\mathrm{cm}^{2} \cdot \sec }\right)=351 \times 10^{22} \frac{P(\text { torr })}{\sqrt{M(g / \text { mole }) T}}
$$

Suppose there is a background pressure of $\mathrm{CO}$ (a typical contaminant) $10^{-6}$ torr, at $300 \mathrm{~K}$. If every molecule of $\mathrm{CO}$ ( $\mathrm{M}=28 \mathrm{~g} / \mathrm{mol}$ ) which hits the surface stays, then the surface flux is $\approx 10^{15}$ molecules per $\mathrm{cm}^{2}$ per second. This is approximately the concentration of atoms on the surface[4]. For this reason, exposing the surface to $10^{-6}$ torr for one second has become a typical process in surface science, and has developed its own unit, the 
Langmuir (L). Frequently experiments will be described by the number of Langmuirs which are used. If the surface need to remain clean for one hour (3600 seconds) then, the pressure must be no higher than $\frac{10^{-6}}{3600}$, or about $5 \times 10^{-10}$ torr.

\section{Single Crystal Metal Surfaces}

The bulk structures of many substances are very regular. If atomic or molecular unit cells repeat periodically within the bulk of a material, the material is crystalline [1]. In the case of rhodium metal, the bulk structure has what is called a face-centered cubic unit cell. This is illustrated in Figure 2-1. The surface structure of a metal single crystal depends on how the plane of the surface cuts through the unit cell. The various orientations are defined by a set of three numbers which depend on where the plane intersects the $x, y$, and $z$ axes of the unit cell [2]. Three different surface structures are used in the majority of single crystal metal studies: the (100) surface, the $(110)$ surface, and the (111) surface. These surface structures are illustrated in Figure 2.2. Each structure has a different type if symmetry. The (100) surface is four-fold symmetric and the atoms on the surface form a square pattern. The (110) surface has rectangular symmetry, and the atoms on the surface are at two distinct heights. The (111) surface has six-fold symmetry in the top layer, but if the second layer is also considered, the two layers have three-fold symmetry.

A systematic notation has been developed to aid the description of adsorbed overlayers on these metal surfaces. These overlayer structures (or, superlattices) are 

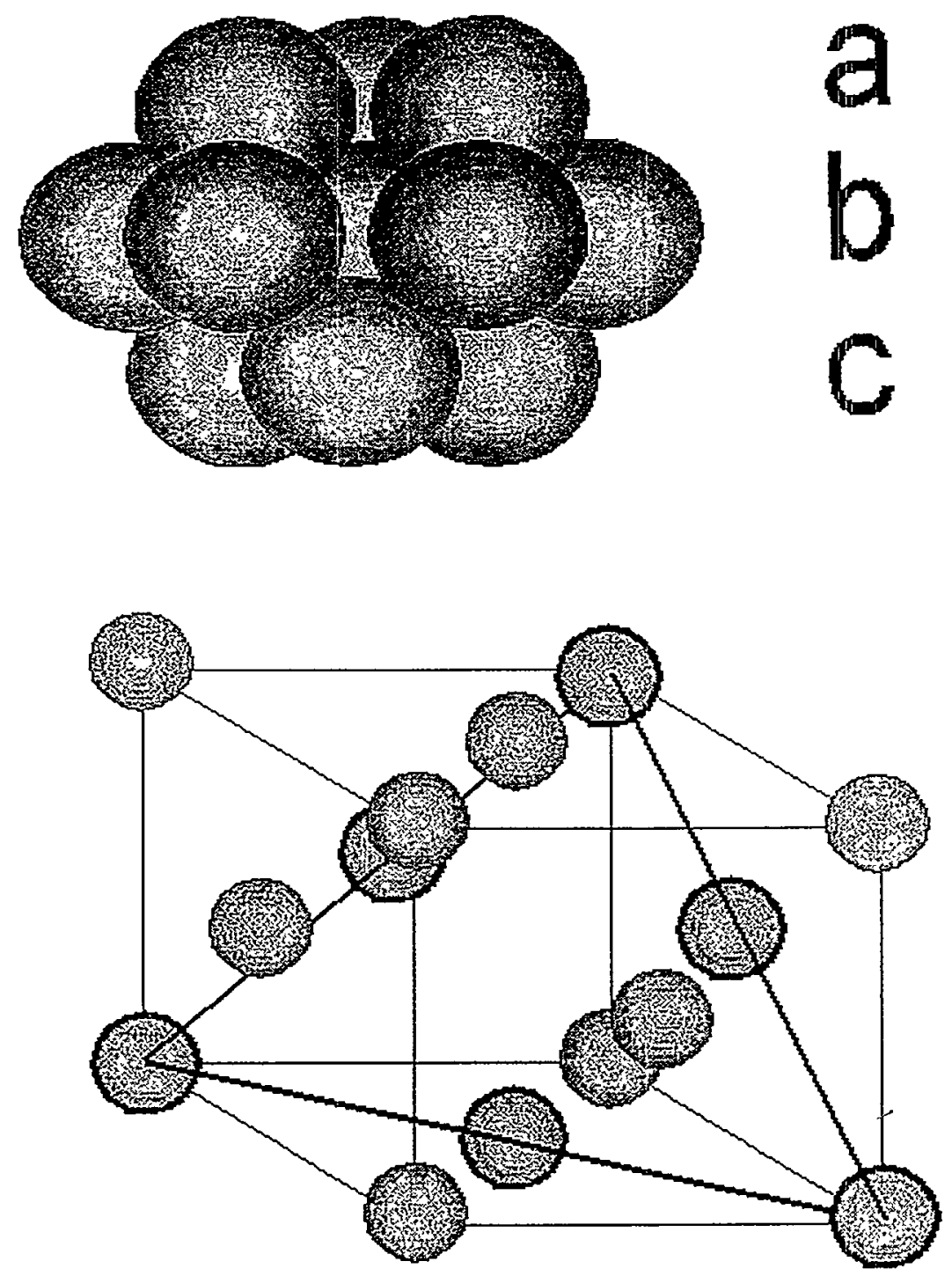

Figure 2-1: Two views of the face centered cubic (FCC) structure of rhodium. Top: There are three different layers of molecules in the FCC structure, labeled abc. In an FCC metal, the set of three layers repeat indefinitely ...abcabcabc... Bottom: The Face Centered Cubic unit cell. The atoms that make up the 111 place are have heavy outlines. 

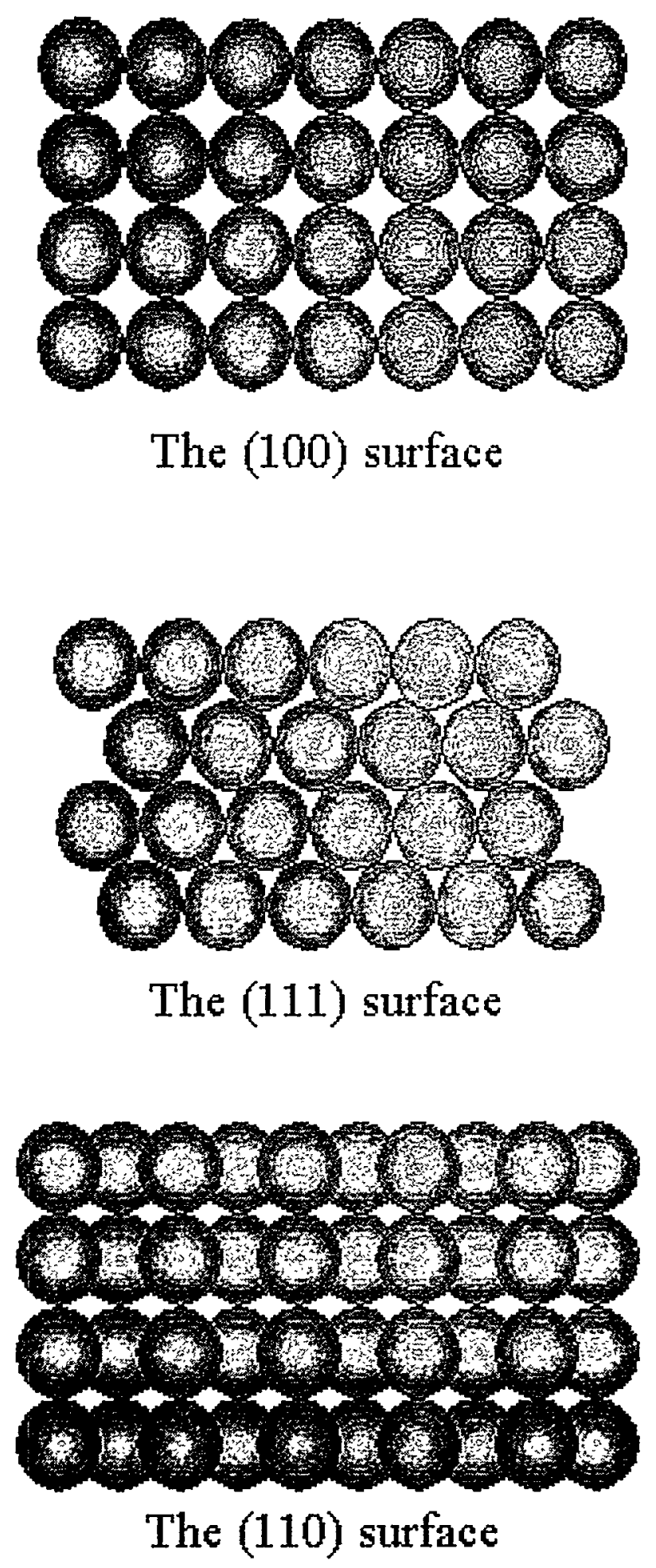

Figure 1-2: The three low-miller-index surface structures. 
given notation based on the relationship between the symmetry and size of their unit cells, using the unit cell of the surface as a reference. A clean surface is said to have a (1x1) structure, so a complete description of a clean single crystal metal surface might be: $\operatorname{Rh}(111)-(1 \times 1)[4]$. If an adsorbate on the surface, say carbon monoxide $(\mathrm{CO})$ has a unit cell twice as large as the metal surface unit cell, then we would describe the structure as $\mathrm{Rh}(111)-(2 \times 2) \mathrm{CO}[5]$. If the unit cell of the adsorbate contains more than one molecule in the unit cell, then that information is also included. In the case of $\mathrm{CO}$ on $\mathrm{Rh}(111)$ there are two structures that have a $(2 \times 2)$ periodicity. One of these structures occurs at low exposures and has one $\mathrm{CO}$ per unit cell, the $\mathrm{Rh}(111)-(2 \times 2) \mathrm{CO}$. The other $(2 \times 2) \mathrm{CO}$ structure occurs at high exposures and has 3 CO's in each unit cell[5]. This structure would be designated $\mathrm{Rh}(111)-(2 \times 2) 3 \mathrm{CO}$. Sometimes the unit cell of the adsorbate is not in the same orientation as the substrate but has its unit cell rotated by some angle with respect to the substrate unit cell. In this case, the angle of rotation is included in the designation. An example would be one of the $\mathrm{CO}$ structures on rhodium which occurs at exposures between the $\mathrm{Rh}(111)-(2 \times 2) \mathrm{CO}$ structure and the $\mathrm{Rh}(111)-(2 \times 2) 3 \mathrm{CO}$ structure[5]. This intermediate structure has a unit cell of $\sqrt{3}$ times the length of the rhodium (111) unit cell and is rotated by $30^{\circ}$. This structure is designated $\mathrm{Rh}(111)-$ $(\sqrt{3} \times \sqrt{3}) \mathrm{R} 30^{\circ}$. If there are more than one type of adsorbed molecule in the adsorbate unit cell, then the numbers and chemical identities of both components are given in the designation. For instance, the coadsorption of benzene and $\mathrm{CO}$ on the rhodium surface with one benzene and 2 CO's in each unit cell is the $\mathrm{Rh}(111)-(3 \times 3) \mathrm{C}_{6} \mathrm{H}_{6}+2 \mathrm{CO}$ structure[6]. Note that the $(3 \times 3)$ unit cell represents the symmetry of the unit cell containing both benzene and $\mathrm{CO}$ and not one of these molecules alone. In some cases, 
these "primitive unit cells" (the unit cell with the smallest possible area is called "primitive") may not give enough information about the internal structure of the overlayer, or the person describing the structure may not want to include the angle of rotation with respect to the substrate. In this case, a unit cell with a larger area may be used. One example of this is the "centered" notation in which defines a unit cell with particular dimensions which has an adsorbate in the center of the unit cell. One typical example of the use of the centered notation is the adsorption structure of one benzene and one $\mathrm{CO}$ in a unit cell on the rhodium surface[6]. In this case, the primitive designation is $\mathrm{Rh}(111)-(\sqrt{7} \times \sqrt{7}) \mathrm{R} 19.1^{\circ}-\mathrm{C}_{6} \mathrm{H}_{6}+\mathrm{CO}$, but is more commonly described as $\mathrm{C}_{6} \mathrm{H}_{6} / \mathrm{CO}: \mathrm{Rh}(111)-\mathrm{c}(2 \sqrt{3} \times 4)$ rect, since there is a unit cell of dimension $(2 \sqrt{3} \times 4)$ which is rectangular and has a benzene in the center. Figure 2-3 shows illustrations of all of the surface structures discussed. 


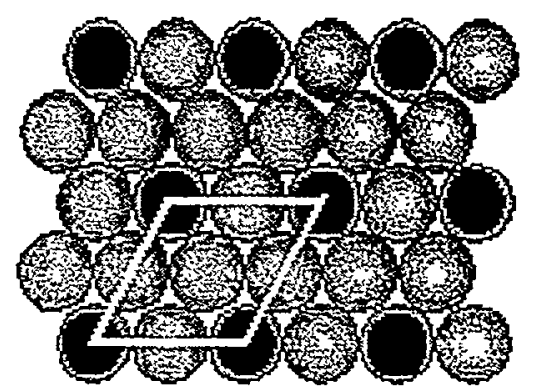

The (2)2) overlayer structure

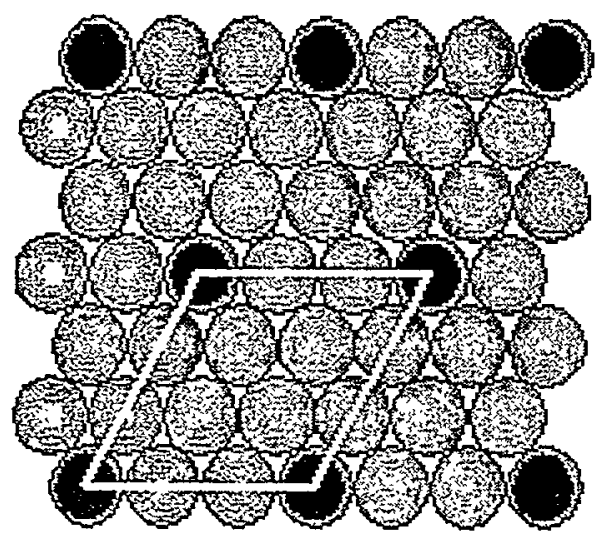

The (3)o overlaver structure

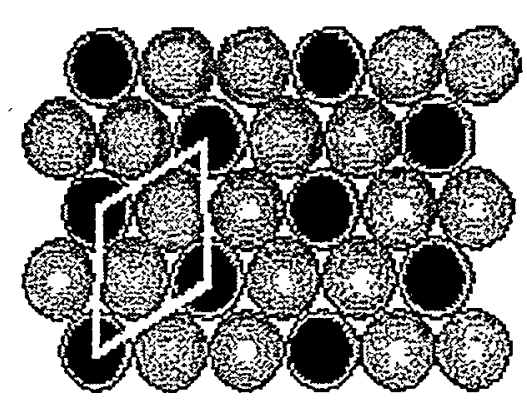

The $(\sqrt{3 \times}, \sqrt{3})$ overlayer structure

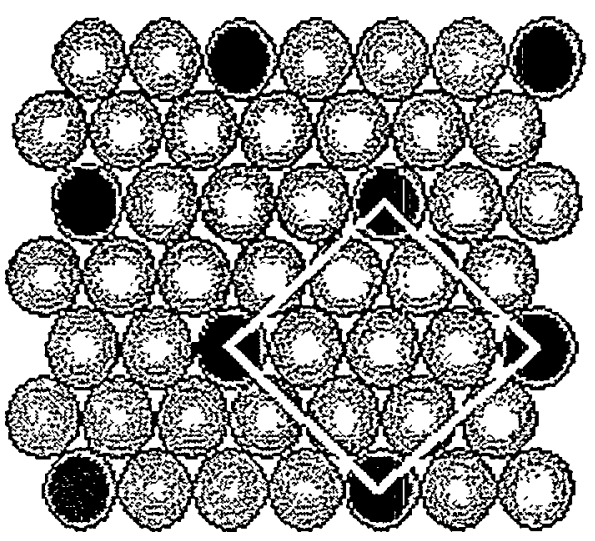

The $(\sqrt{7} \times \sqrt{7})$ overlayer structure

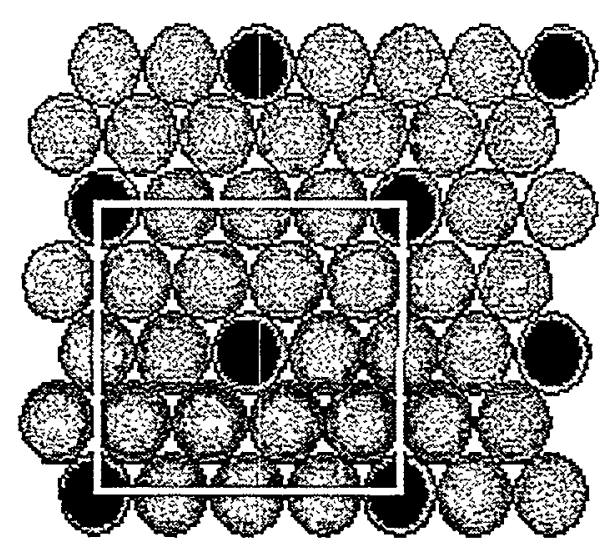

The $(2 \sqrt{3} \times 4)$ overlayer structure

Figure 2-3: Five examples of the notation used to describe ordered overlayers. 
References

[1] H. Ibach, and H. Lüth; Solid-State Physics, $2^{\text {nd }}$ Ed.; Springer-Verlag; New York, 1995; p. 19.

[2] C Kittel, Introduction to Solid State Physics, $7^{\text {th }}$ Ed; John Wiley and Sons; New York; 1996; p. 14.

[3] F.O. Goodman, "Scattering of Atoms and Molecules by Solid Surfaces", in: Critical Reviews in Solid State and Materials Sciences, Vol. 7, R. Vanselow, ed., CRC Press, Boca Raton, Fl, 1977.

[4] G. A. Somorjai, Introduction to Surface Chemistrv and Catalysis. John Wiley and Sons, New York, NY, 1994.

[5] D.G. Castner, B.A. Sexton, and G.A. Somorjai, Surf. Sci. 71 (1978) 519.

[6] R.F. Lin, R.J. Koestner, M.A. Van Hove, and G.A. Somorjai, Surf. Sci. 134 (1983) 161. 


\section{Chapter 3: Experimental Apparatus}

\section{Introduction}

A state-of-the-art UHV vacuum chamber includes a variety of instruments.

Typically, the cleaning and characterizing of the sample surface require a number of techniques, including Auger Electron Spectroscopy (AES), Mass Spectrometry (Mass.

Spec.), Electron Gun Bombardment, and Low Energy Electron Diffraction (LEED).

Maintaining a UHV environment involves the use of mechanical pumps, tubomolecular pumps, and ion pumps, as well as an ion gauge to measure the pressure in the chamber. A sample manipulator is used to raise and lower the sample as well as rotate the sample around to face each of the instruments mounted on the chamber. Finally, valves are needed which maintain Ultra High Vacuum but allow sample gassed to be dosed into the chamber. This chapter will discuss some of the more important instruments which are used in surface science and will outline the theory of their operation.

A diagram of the vacuum chamber used in the experiments is given in Figure 3-1. The electron guns and the LEED apparatus require alignment to the sample so that the electron beams focus on the sample. The Mass. Spec. and the STM do not have electron 
beams to align, but they are positioned around the chamber so that the sample can be manipulated to be within a few centimeters of the instruments. In the STM's case this is so that the sampled can be easily transferred from the sample holder to the STM stage.

For the Mass. Spec., the alignment is necessary so that the sample can be placed close to the ionizer. This reduces the amount of background gasses which impinge on the ionizer and allows the determination of species leaving the sample, usually when the sample is heated.

\section{Sample Preparation}

The $\mathrm{Rh}(111)$ single crystal was cut from a single crystal boule and aligned with $\mathrm{x}$ ray diffraction techniques. The sample was then polished using diamond paste of particle size as small as. 25 microns. The sample was then mounted on a T-shaped holder using strips of tantalum foil which are spot welded onto the steel face of the sample holder. The mounted sample and the sample holder attached to the manipulator are shown in Figure 3-2. To further clean the Rh crystal, it is bombarded with Ar ions. These are created by a using high-energy electron beam $(1500 \mathrm{eV})$ which ionizes Ar atoms in the gas phase. The momentum of the electrons which interact with the Ar atoms cause them to impinge on the surface. Surface atoms, both Rh and impurities on the surface, are removed by 


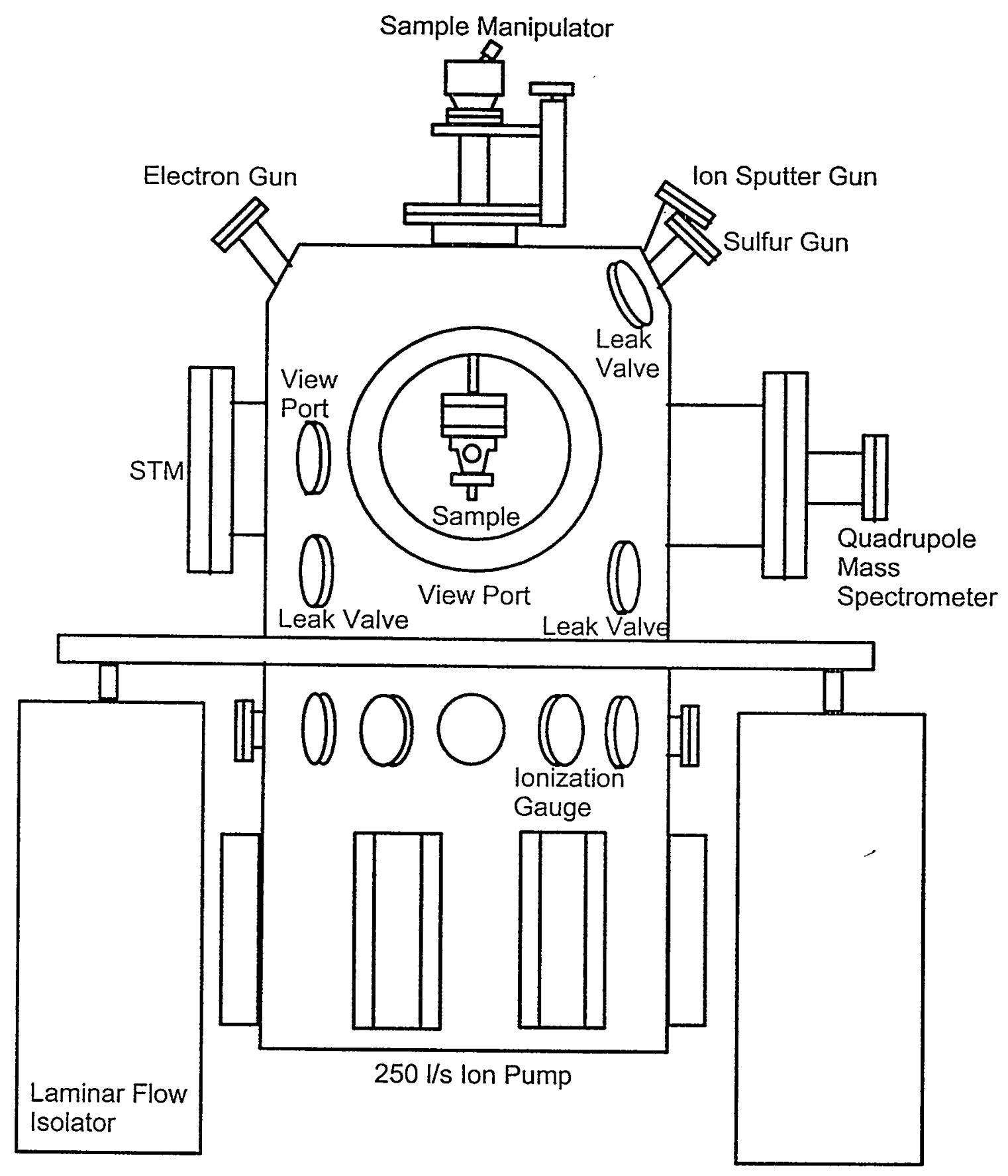

Figure 3-1: A schematic diagram of the UHV chamber. 


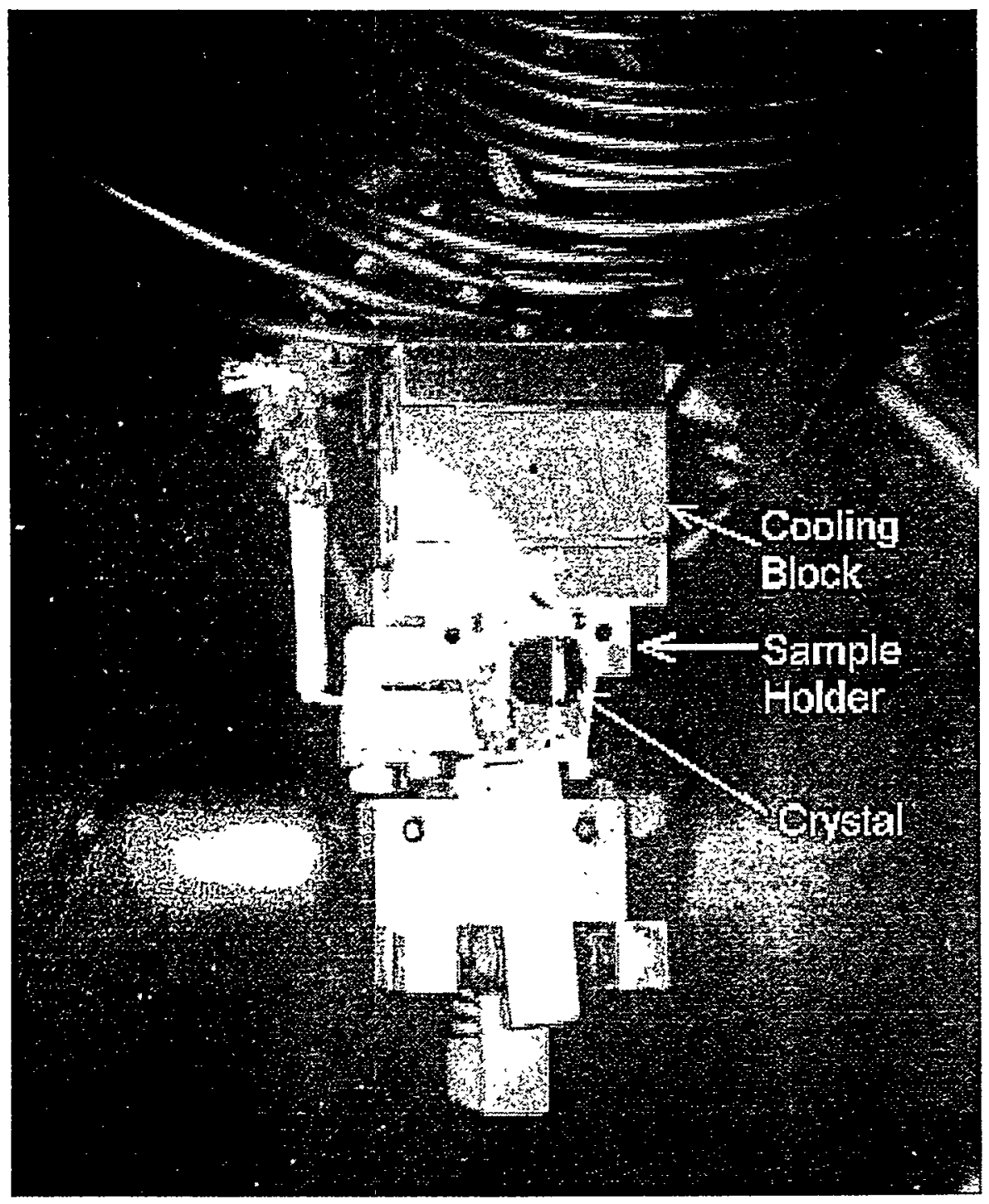

Figure 3-2: A photograph of the sample holder with the sample in place. 
momentum transfer from the Ar ions. In order to counteract the roughening effect of the ion bombardment process, the sample is annealed at $2700 \mathrm{~K}$ for $20-30 \mathrm{~min}$.

If the concentration of the impurities on the surface is low, then annealing the surface in a reactive gas can remove the contaminants with a smaller amount of damage to the surface than is typical in the Ar ion bombardment process. The removal of Carbon from the surface can be achieved by annealing the sample to $500 \mathrm{~K}$ in $5 \times 10^{-8}$ torr of $\mathrm{O}_{2}$ gas for $10-15 \mathrm{~min}$. The $\mathrm{O}_{2}$ gas is then removed form the system and the annealing is continued in vacuum to remove any adsorbed oxygen and to allow the surface to recover from any damage done during the carbon removal process.

\section{Auger Electron Spectroscopy}

One of the techniques used to determine the chemical identity of atoms at the surface of a sample is called Auger Electron Spectroscopy (AES). A diagram of the Auger process is shown in Figure 3-3. When a surface is bombarded with high energy electrons, some electrons interact with inner shell electrons in the atoms in the surface layers of the metal, causing the ionization of the atom by ejection of the core electron.[1] An electron in a higher energy orbital can then relax into the empty core hole. The excess energy given off by this process can eject a second electron, called an Auger electron, which can be collected and analyzed. Because the energy of this Auger electron is dependent upon the energy of the three energy levels, an analysis of the Auger electrons is able to distinguish the chemical composition of the atoms on the surface. The technique is surface specific because the energy of the Auger electrons produced is usually on the order of $50-1000 \mathrm{eV}$. Since Auger electrons produced in the 

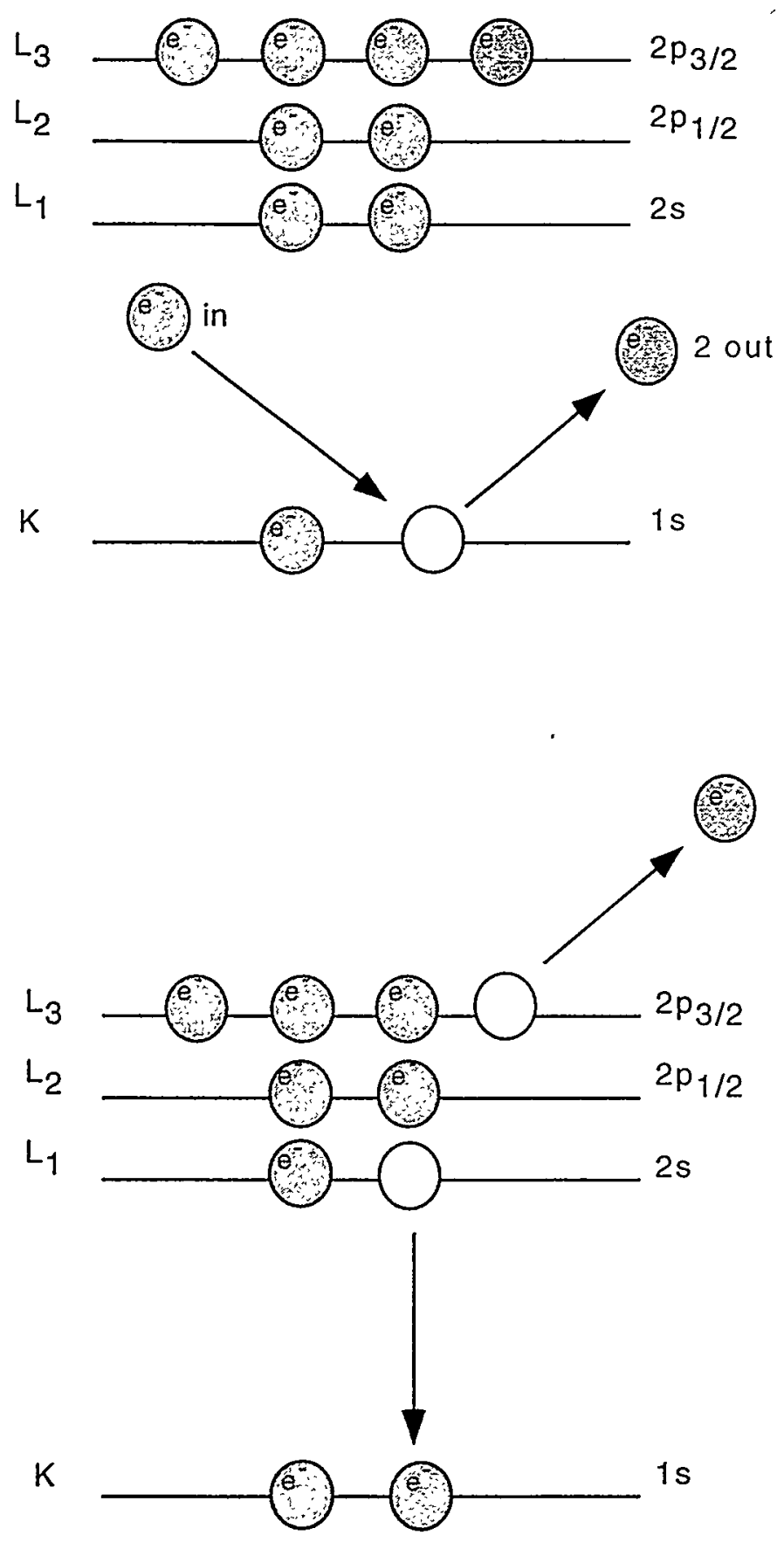

Figure 3-3: A diagram showing the two steps in the Auger process. Top: A core electron is ejected by an incoming high-energy electron. Bottom: An electron drops to the low lying empty state and another electron is ejected. 
bulk travel through the more of the metal than electrons produced in the surface layers, they are less likely to escape the metal. Also, the surface sensitivity can be enhanced by allowing the electrons to impinge on the surface at a grazing incidence, which prevents many of the electrons from penetrating into the bulk, thereby enhancing the number of surface exitations [2].

The Auger electrons are collected by a Retarding Field Analyzer (RFA) which is also used as the LEED optics for the system. In order to reduce the background signal, the derivative of the number of electons as a function of energy, $\mathrm{dN}(\mathrm{E}) / \mathrm{dE}$, is collected. This is accomplished by modulating the voltage to the RFA grids and then detecting the second harmonic of the signal with a lock-in amplifier[3]. A diagram of the electronics is shown in Figure 3-4.

The signal collected is recorded and analyzed to determine the chemical composition of the surface layers. A set of typical Auger specta are shown in Figure 3-5. The rhodium metal shows a characteristic set of three peaks at $220 \mathrm{eV}, 264 \mathrm{eV}$ and $302 \mathrm{eV}$. Other common peaks in an Auger spectrum include a carbon peak at $272 \mathrm{eV}$. This often shows as a shoulder in the $\mathrm{Rh} 264 \mathrm{eV}$ peak. Determining that a rhodium surface is completely free of carbon is therefore very difficult since a small carbon peak would be obscured by the $264 \mathrm{eV}$ rhodium peak. Oxygen has an Auger peak at $510 \mathrm{eV}$. Sulfur is also a common contaminant, and its Auger peak is at $150 \mathrm{eV}$. 


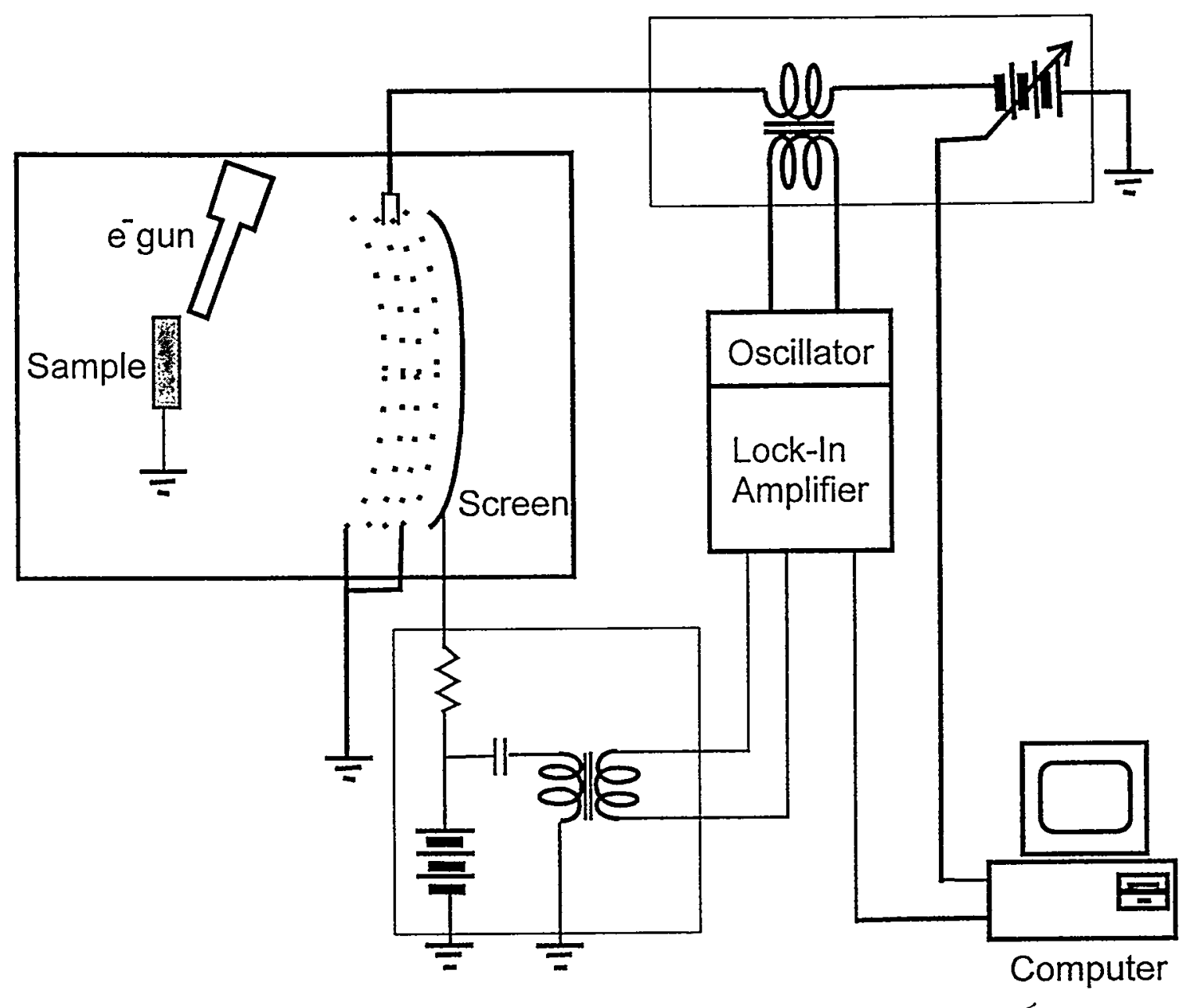

Figure 3-4: A schematic diagram of the Auger electronics. 


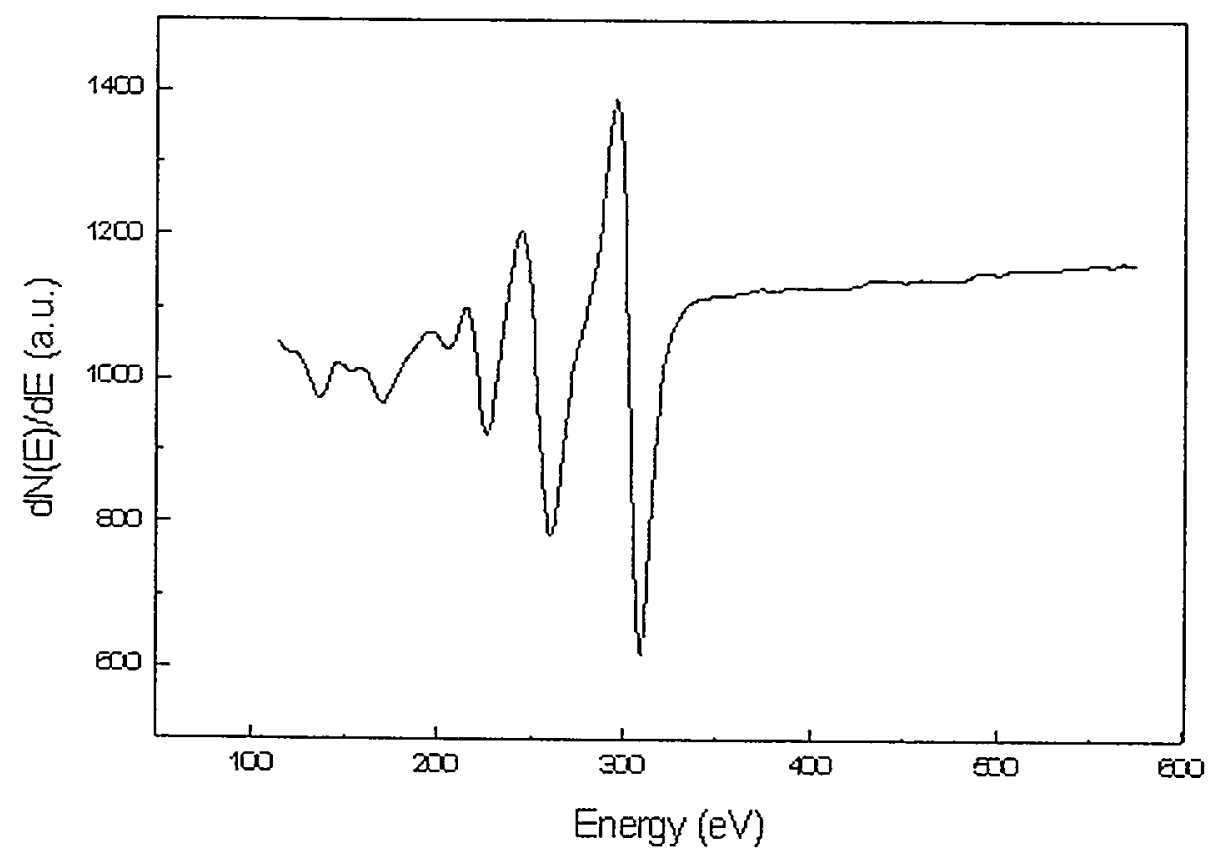

Figure 3-5: An example of an Auger spectrum of rhodium. The three rhodium peaks are evident, as well as possible sulfur contamination. 


\section{Low Energy Electron Diffraction}

Low Energy Electron Diffraction is a technique to use the information contained in scattered electrons to determine the structure of the surface. At a very basic level, LEED can be used to determine the symmetry of the surface and surface overlayer by simply inspecting the patterns formed by electrons as they are backscattered from the surface. The more advanced interpretation of scattering data allow the determination of surface structure to an accuracy of better than tenths of Ångstroms.

There are a number of texts which explore the fundamentals of LEED Theory $[4,5]$, and a thorough discussion is out of place here, as the experiments in this work only use LEED as a qualitative technique. The LEED apparatus used in these experiments consists of an electron gun which produces a monoenergetic beam of electrons. After these electrons are back-scattered off of the sample, they encounter a set of grids in front

of a phosphorescent screen. The first screen is grounded so that there is no electric field between this grid and the sample. The second grid is biased to the same voltage as the electron beams so that only elastically scattered electrons are able to pass. The third grid is positively biased to accelerate the electrons onto the phosphorescent screen. This pattern is inspected visually and often recorded by photography or with a video camera connected to a computer. A schematic diagram of this experimental set-up is shown in Figure 3-6.

The information obtained by visual inspection of the LEED pattern includes the geometric symmetry of the unit cell of both the metal surface and the adsorbed overlayer, 


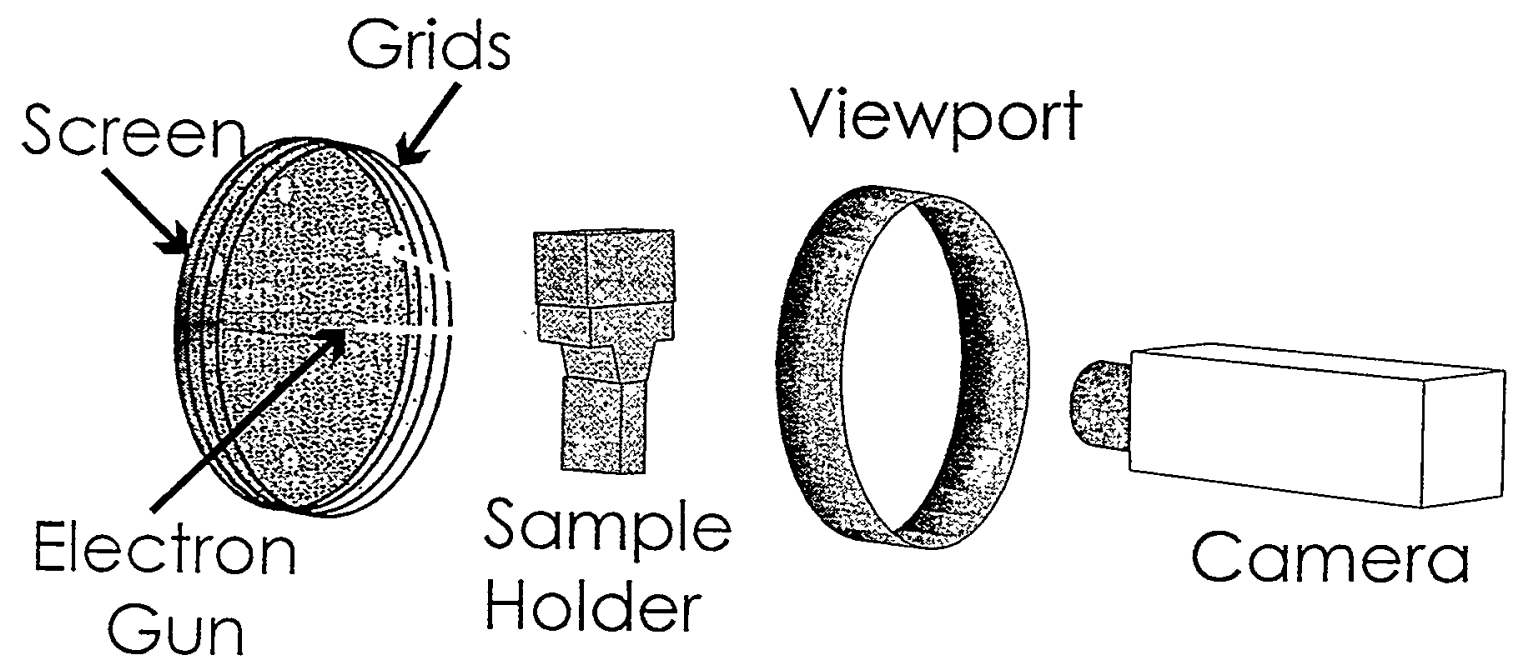

Figure 3-6. A drawing of the arrangement of the LEED system components. 

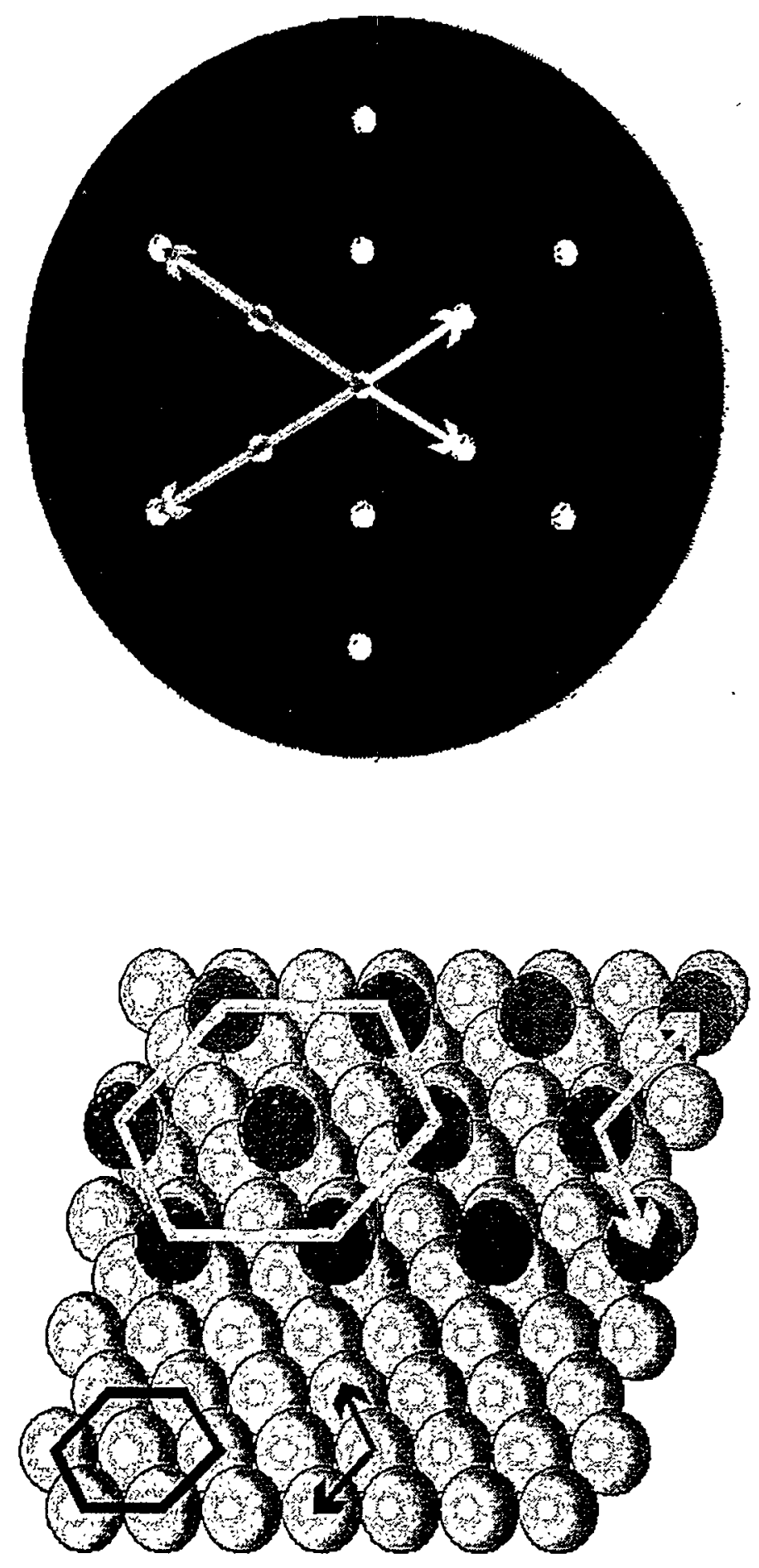

Figure 3-7. The corresponance between the LEED pattern and the Surface structure. Top: A simulated LEED pattern. Bottom: The surface which would yield such a pattern. 
as well as the relative sizes of these unit cells. A diagram of how the surface structure correlates with the LEED pattern is shown in Figure 3-7. If a surface unit cell has hexagonal symmetry, the LEED pattern will also have the same symmetry. The sizes of the unit cells have a reciprocal relationship, i.e. If the size of the overlayer unit cell is twice as large as the surface unit cell, then the spots on the LEED screen will be one-half the distance form the center of the screen. However, if there is any difference in the relative orientations of the two overlayers (a rotation by $30^{\circ}$, for instance), then there will be a corresponding rotation of the spots in the unit cell. 


\section{References}

[1]. D.P. Woodruff and T. A. Delchar; Modern Techniques of Surface Science;

$2^{\text {nd }} \quad$ Ed.; Cambridge University Press; 1994; p. 171.

[2] P. W. Palmer, Appl. Phys. Lett 13, 183 (1968).

[3] G. Ertl and J. Küppers, Low Energy Electrons and Surface Chemistry,

Weinheim: Verlag Chimie; 1974.

[4] M.A. Van Hove and S.Y. Tong; Surface Crystallography by LEED; SpringerVerlag; Berlin; 1979.

[5] M. A. Van Hove, W.H. Weinberg, and C.-M. Chan; Low-Energy Electron Diffraction; Springer-Verlag; Berlin; 1986. 


\section{Chapter 4: Scanning Tunneling Microscopy}

\section{Introduction}

The Scanning Tunneling Microscope (STM) is a tool to view the electron distribution in a molecule or conglomeration of molecules. While not an optical image, as the term microscope may suggest, the pictures produced by an STM are still a spacial representation of matter at an atomic length scale. Developed by Gerd Binning and Heinrich Rohrer at the Beginning of the 1980's it was quickly recognized as a powerful tool to probe the structure of metal surfaces[1]. The impact it had on the physics community may be gauged by the fact that from the first measurements taken on March 16,1981 , it was less than six years before Binning and Rohrer were awarded the Nobel Prize in Physics on December 8, 1986[1].

In the past two decades, the STM has become a widely used tool for the imaging of surfaces of solids, liquids and thin films. The original experiments examined the surface of $\mathrm{CaIrSn}_{4}[2]$, and was also used to study surface reconstructions, such as the (7x7) reconstruction of the silicon (111) surface [3] and the $(2 \times 1)$ reconstruction of the gold (110) surface [4]. Other groups have used the STM to investigate semiconductor 
surfaces, such as GaAs[5], adsorbed monolayers on metal surfaces[6] and the adsorption of molecules onto electrodes in electrochemical cells[7]. With the wide range of possible experiments which are open to the users of the STM, the number of groups using them grew quickly through the end of the 1980's. The number of scientists participating in STM conferences was less than 100 in 1985 , but had increased by an order of magnitude to more than 1000 by $1991[8]$. Certainly, this trend has continued as the importance of nanoclusters and sub-micron systems has grown.

\section{Theory of Operation}

An STM operates by bringing a sharp metal tip to within a few Ångstroms of a conducting material. When the tip is very close to the sample, electrons are able to tunnel from one to the other depending upon the relative positions of the Fermi levels of the tip and the sample. The number of these electrons is measured as a tunneling current. Specialized electronics and a computer then use this current to create a map of the surface.

The fundamental principles which allow the STM to operate are quantum mechanical in nature. When two metal objects are brought close together, there is a electric potential barrier which corresponds to the energy needed to remove one electron from the metal and pass to the other metal. In general, electrons in metals at room temperature do not have enough energy to overcome this potential barrier. In classical physics, electrons which do not have enough energy to overcome a potential barrier have no probability of getting form one side of the barrier (the metal) to the other side (the tip). However, in quantum mechanics there is a finite probability for the electron to pass from 
one metal to the other due to the wave nature of the electron. In the case of the STM, the barrier between the two conductors - the tip and the sample- is a vacuum. For this reason the STM is said to be based on the theory of the metal-vacuum-metal tunneling, or just vacuum tunneling for short. The vacuum tunneling probability is actually easy to calculate in a one-dimensional form with a treatment called Wentzel-Kramer-Brilloun (WKB) theory, and basic treatments of the theory of the STM are based on the principles of this WKB theory.

A general representation of a tip-vacuum-metal energy diagram is given in Figure 4-1. The energy levels of the tip metal with the filled levels shaded, and the Fermi level labeled $E_{F}$ is on the left of the diagram and the energy levels of the sample are displayed in a similar manner on the right of the diagram. Note that the two Fermi levels are at different energies. This is usually caused by the application of an external "bias" voltage. If the bias voltage is applied to the sample and is positive, then the Fermi level of the sample is lowered. This is the situation shown in Figure 4-1. In this case, the electrons from the filled energy levels of the tip tunnel into the empty energy levels of the sample. If the bias were negative on the sample, then the electrons from the filled, higher energy levels would tunnel into the empty levels of the tip. These two bias possibilities allow the STM to investigate both the filled and empty states of the sample. In some cases the images from filled and empty states will be very different, such as in GaAs. In other cases, they will be very similar, as the images of clean metals tend to be. The distance between the sample and the tip is represented by " $\mathrm{z}$ ", and the vacuum potential is the line between the tip and sample work functions, $\Phi_{\text {tip }}$ and $\Phi_{\text {sample }}$, respectively. The electron which is tunneling from the tip to the sample is represented by the sinusoidal function 


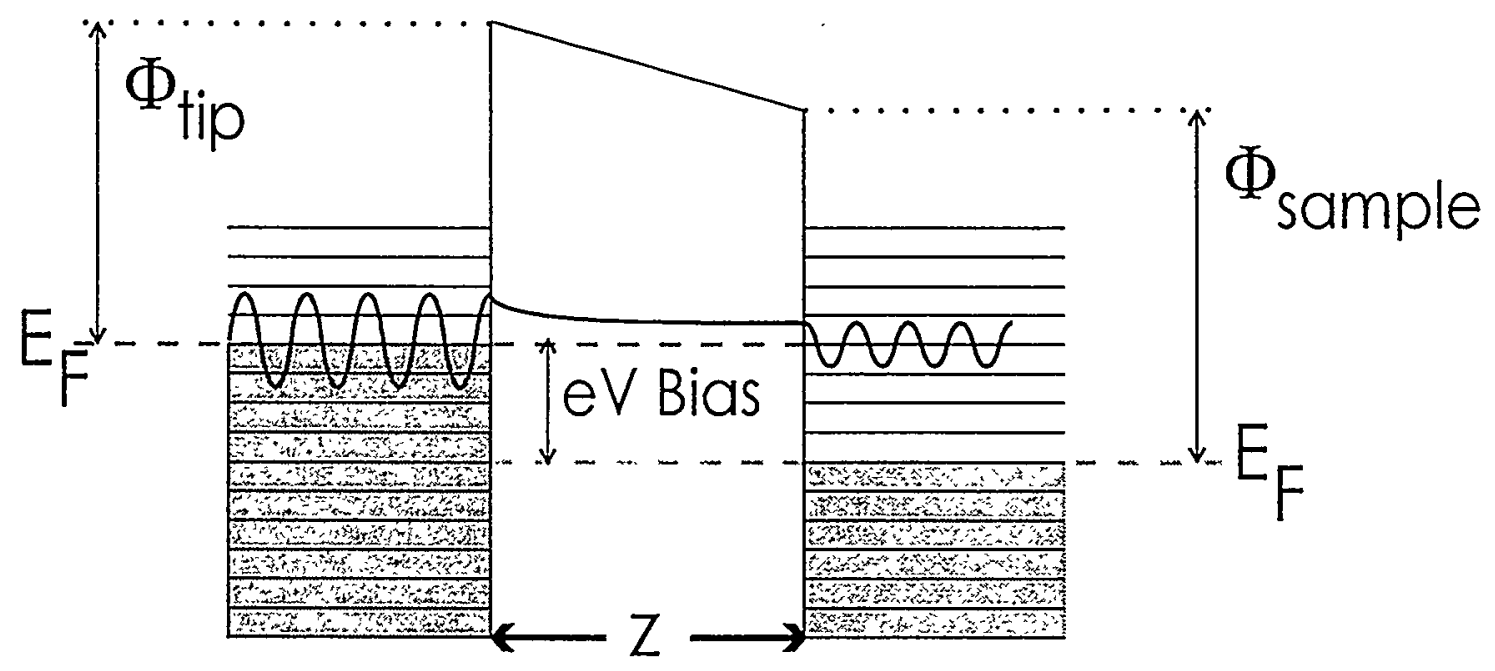

$$
I \propto \exp \left(-\bar{\phi}^{1 / 2} \cdot z\right)
$$

Tip
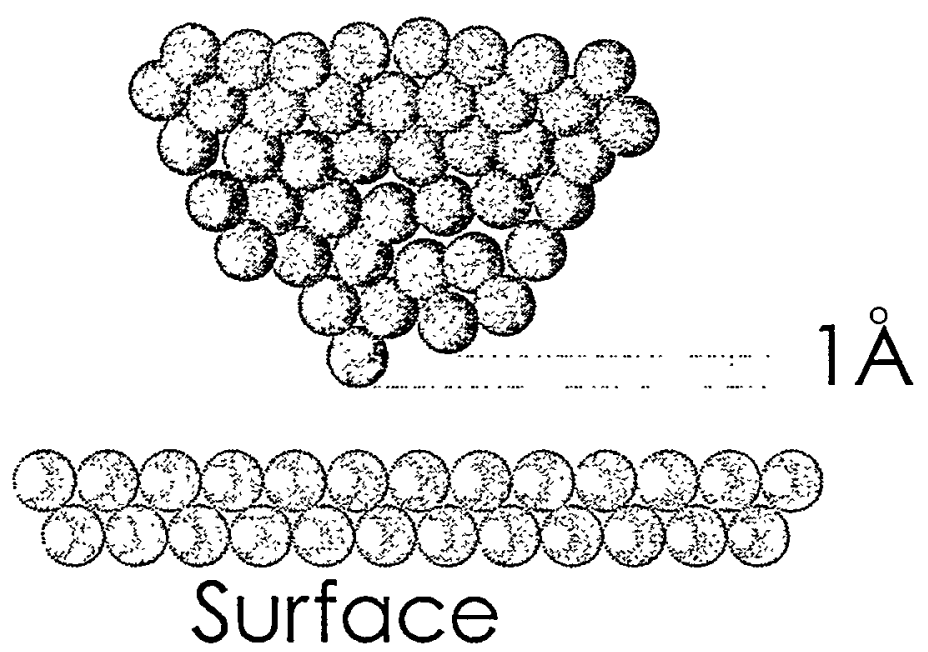

Figure 4-1: Top: A representation of the tip-vacuum-metal energy level diagram. Bottom: An atomic view of the tip-vacuum-metal junction. 
wavefunction which decays exponentially within the tunneling barrier. While the amplitude of the wave function changes from the tip to the sample, the energy of the electron is conserved.

The total tunneling current is caused by the movement of electrons back and forth from the tip to the sample and vice-versa. However, the filled-to-filled tunneling tends to cancel out, and the only measurable contribution to the tunneling current is from the filled-to-empty tunneling processes. The equation for the total tunneling current, which is derived from WKB theory is given below the energy level diagram. It is exponentially dependent on the distance between the tip and the sample, $z$, and the average vacuum potential, $\bar{\Phi}$.

For a change in separation of one Ångstrom, the tunneling current changes by almost a factor of ten. This sensitivity to the distance between the tip and the sample is the crucial effect which is used to take images with the STM. Figure 4-1 also shows a model tip and surface. There are a large number of atoms in the tip which can be involved in the tunneling, but only the closest contributes significantly to the tunneling current. Because of this, STM tips are not required to be sharp on the Angstrom scale, since there only needs to be one atom which is one Ångstrom closer to the surface to have tunneling from effectively a single atom.

The STM can be used to image in a number of different ways based on how the tunneling current is used. Figure 4-2 illustrates the two most common tunneling conditions, constant height imaging and constant current imaging. In the constant height mode, the STM tip is kept at a fixed difference from the surface while it is moved over 


\section{Constant Height}
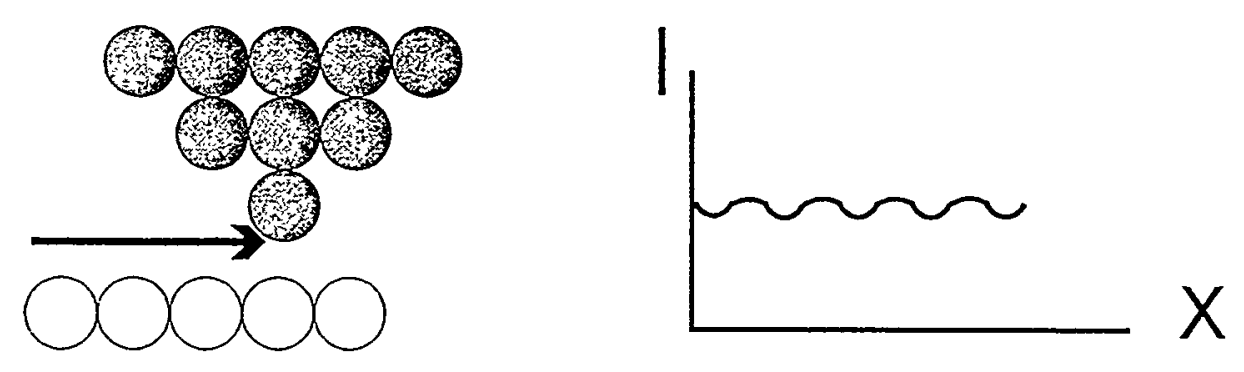

\section{Constant Current}
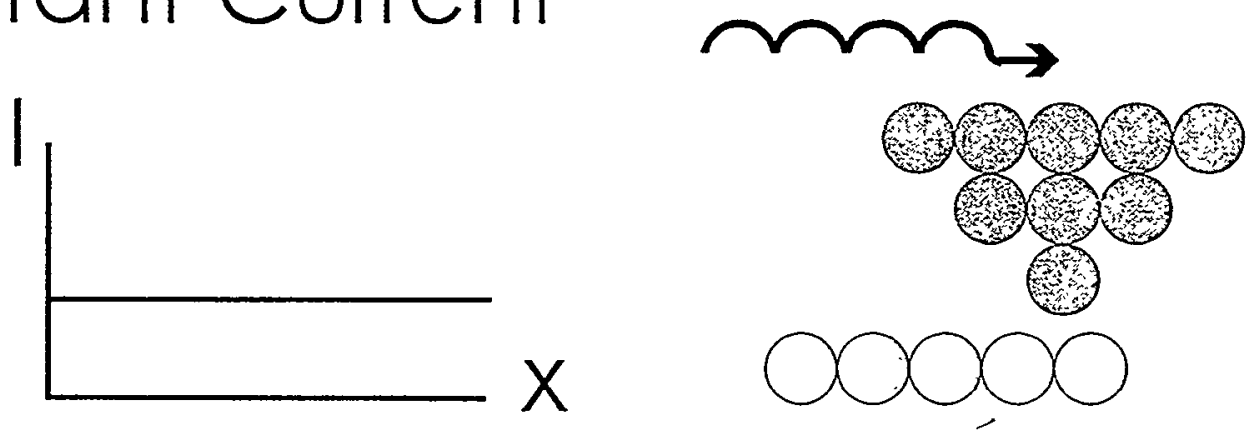

Figure 4-2: An illustration of the two main types of operation of an STM. Top: Constant height mode, where the tip is held at a constant distance from the surface and the measured current reflects the topography. Bottom: Constant current imaging where an electronic feedback loop maintains constant current and the z-displacement of the tip reflects the surface topography. 
the surface. The tunneling current is then directly used to determine the topography of the surface, since the current will increase when the atoms on the surface are closer to the tip, and decrease when they are farther away from the tip. This mode is often used when the surface is very flat. The second way to take images with the STM is the constant current mode. In this case, the tunneling current is used in a feedback loop to detect slight changes in tunneling current. The tip is then moved closer to the surface or farther away, depending on whether the tunneling current has decreased or increased. The position of the tip is then used to create a topographic image of the surface, rather than using the tunneling current directly. This keeps the tip at a constant separation from the surface and allows the STM to image very rough or stepped surfaces easily.

\section{Instrument Design}

Figure 4-3 is a picture of the microscope used for the experiments described in later chapters. The "scan head" of the STM, the portion of the instrument which holds the tip and scans it across the surface is outlined in the schematic drawing at the bottom of the figure. The scan head consists of two cylindrical piezoelectric tubes. A piezoelectric material, in this case lead titanate-zirconate (Stavely EBL 3 PZT), deforms slightly when a voltage across it. The outer piezoelectric tube is used to move the sample car along two anodized aluminum guides to within a few millimeters of the tip. The displacement of the sample is caused by a "jerk" mechanism, where the piezoelectric tube slowly contracts slightly to bring the sample closer to the tip. Then the outer tube is allowed to quickly return to its equilibrium length. If this second motion is quick enough, then the sample car is not moved and remains a little closer to the tip. This is the 

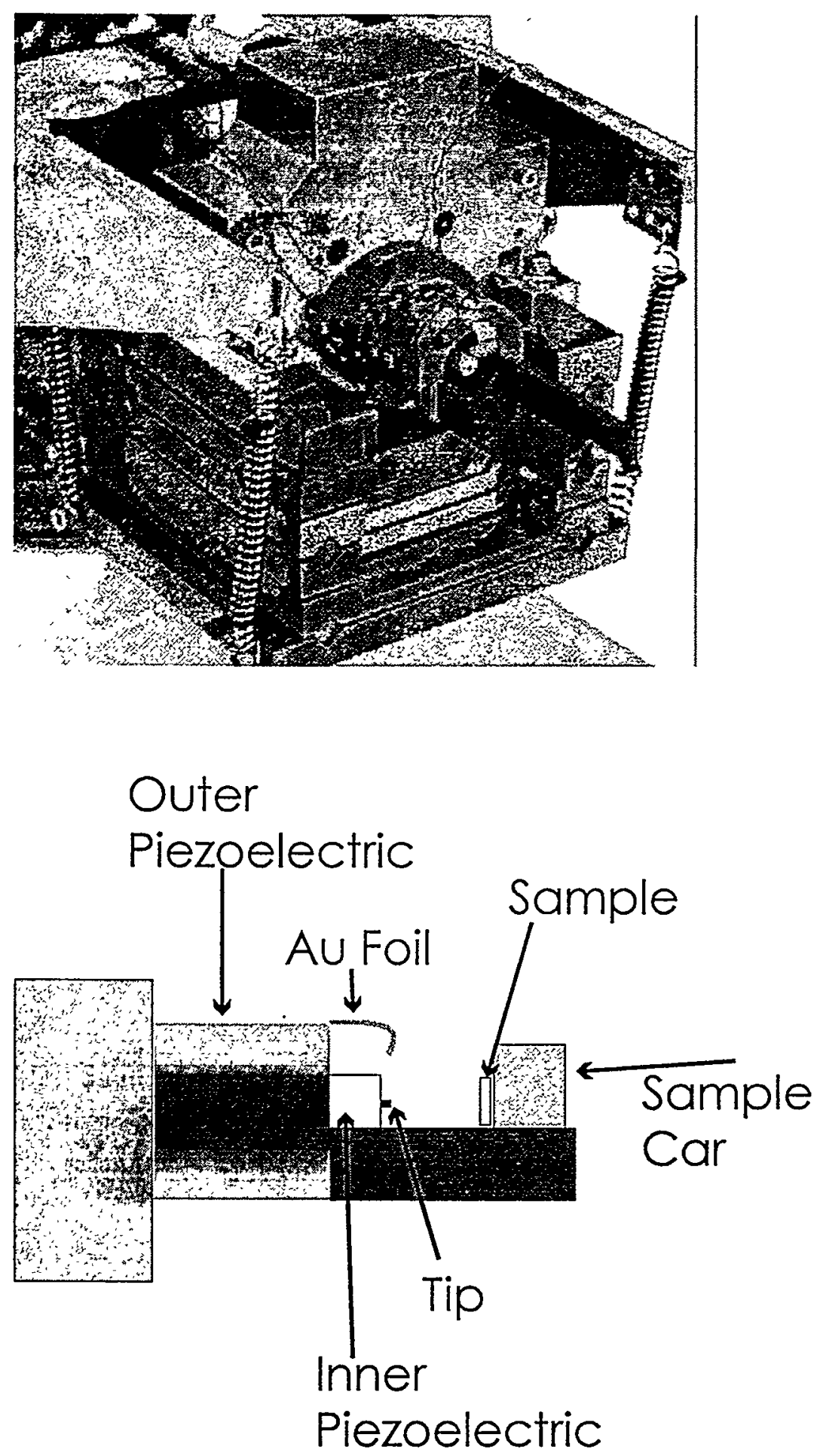

Figure 4-3: Top: A photograph of the STM. Bottom: A schematic diagram of the two scannning heads and the position of the sample. 
same mechanism used in the trick where a magician pulls a tablecloth out from under a set of dishes. If the motion is slow, the dishes move with the cloth, but if the motion is quick enough, the magician can move the tablecloth and leave the dishes in place. During the motion, the electronics test for the magnitude of the sample current. When the current reaches a threshold limit, then the outer piezoelectric motion stops. The electronics can be used to manually move the inner piezoelectric tube close enough to the sample to begin scanning. This distance is generally on the order of $5-10 \AA$ Angstroms, depending on the desired tunneling current and bias voltage. The inner piezoelectric tube is divided into four sections. When a voltage is applied to the top and bottom sections, the tip moves up or down (in the "y" direction) depending on the sign of the applied voltage. The use of the other two sections allow the tip to move in the left-right ("x") direction. If the tip needs to be moved closer to or farther from the sample, as is necessary in the constant current mode, the motion is caused by applying a voltage to all four sectors simultaneously, which has the effect of lengthening or contracting the piezoelectric tube. The tip receptacle consists of a machined ceramic with a small tube in the center, which is epoxied to the end of the inner piezoelectric tube. The tip is a metal $(90 \% \mathrm{Pt}, 10 \% \mathrm{Rh})$ wire inserted into the tube of the tip receptacle. The tip is held in place by slightly bending the tip so that it is wedged into the tube. The final piece of the scan head is a small piece of gold foil, which is pressed against the sample car when it is close to the tip. This is used to apply the bias voltage to the sample.

The STM needs to be isolated from ambient vibrations so that the tunneling gap can remain stable at a few Angstroms. This is accomplished with three different techniques: extension springs, vibration springs, and compressed air floatation 
stages. The STM head and the stainless steel plates which form the body of the STM are suspended from the UHV flange by four extension springs. The springs should have as small a spring constant as is possible given the constraint that the STM must be at a certain height with respect to the sample manipulator. The stainless steel plates which are separated by compression springs. These springs are pieces of Viton, a low vapor pressure rubber. These Viton springs act as spacers to separate the metal plates and dampen vibrations. The whole stainless steel chamber is mounted on a set of compressed air legs which isolate the chamber from ambient building vibrations. These legs support the chamber with compressed air so that there is no mechanical contact between the chamber and the floor of the room. This reduces the low frequency $(<60 \mathrm{~Hz})$ noise associated with the movement of the building. 


\section{References}

[1] Binning and Rohrer, Rev. Mod. Phys., 59, no.3, 1987, p615.

[2] Binning, Rohrer, Gerber, and Weibel, Phys. Rev. Lett., 49, 1982, p57.

[3] Binning and Rohrer, Surf. Sci., 126, 1983, p236.

[4] Binning, Rohrer, Gerber, and Weibel , Surf. Sci., 131, 1983, pL379.

[5] Feenstra and Stroscio, Phys. Rev. Lett., 59, 1987, p2173.

[6] Chiang, Wilson, Mate, and Ohtani, J. Vac. Sci. Technol. A, 6, 1988, p386.

[7] Trevor, Chidsey, and Loiacono, Phys. Rev. Lett., 62, 1989, p929. 


\section{Chapter 5: Carbon Monoxide}

\section{Introduction}

The adsorption of carbon monoxide on noble metal surfaces is a well-studied reaction. The metal surface / CO system is important in the catalytic production of methanol from methane and the oxidation of $\mathrm{CO}$ to $\mathrm{CO}_{2}$ in automobile catalytic converters. It is therefore surprising that after the considerable effort devoted to the study of $\mathrm{CO}$ adsorption, the molecular details of the reaction are still not well understood.

Carbon monoxide forms several ordered surface structures on the (111) crystal face of rhodium as the coverage is increased by increasing the $\mathrm{CO}$ pressure over the surface. Low energy electron diffraction (LEED) - surface crystallography studies determine the location of molecules on the metal surface and carbon-oxygen bond distances at low ambient pressures. Scanning tunneling microscopy (STM) is also used to characterize the same CO surface structures that were studied by LEED. In addition, STM can be employed at high $\mathrm{CO}$ pressures to monitor the CO surface structures that form in up to one atmosphere of $\mathrm{CO}$ pressure over the $\mathrm{Rh}(111)$ surface. At these high 
pressures the $\mathrm{CO}$ surface coverage is in equilibrium with gas phase $\mathrm{CO}$ so that the molecular $\mathrm{CO}$ surface structures are those characteristic of the adsorption isotherm. Vibrational spectroscopy techniques, HREELS at low CO pressures, infrared spectroscopy at high pressures and SFG at both low and high CO pressures also contribute greatly to our understanding of $\mathrm{CO}$ bonding to $\mathrm{Rh}(111)$ and how this changes with $\mathrm{CO}$ pressure.

The CO surface structures that have been seen by LEED or STM as the CO coverage is increased are the low coverage $(2 \times 2),(3 \times 3) \mathrm{R} 30^{\circ},(2 \times 1),(\sqrt{ } 7 \times \sqrt{ } 7) \mathrm{R} 19^{\circ}$ and the high coverage $(2 \times 2)-3 \mathrm{CO}$. In this paper we review what is known about each $\mathrm{CO}$ surface structure. Several of the surface structures that were detected by STM are reported for the first time. There is evidence of surface restructuring of the $\mathrm{Rh}(111)$ crystal face with increasing CO pressure (coverage) as shown by SFG. We have not found evidence so far for the formation of rhodium carbonyl species on the (111) crystal face, a stable molecule that can be readily produced on small rhodium particles in solution or in the gas phase from other rhodium compounds.

In the case of Rh(111), previous work has produced a number of models for the structure and adsorption site of the CO molecules. The first structural results appeared nearly 30 years ago, when Grant and Haas [1] found by Low Energy Electron Diffraction (LEED) that CO formed a $(2 \times 2)$ structure. Thiel et al. [2] found two structures with $(\sqrt{3} \times \sqrt{3}) \mathrm{R} 30^{\circ}$ and $(2 \times 2)$ periodicity at coverages of 0.33 and 0.75 monolayers (ML). A hint of a lower coverage $(2 \times 2)$ structure was also mentioned in their work. High resolution electron energy loss spectroscopy (HREELS) studies [3] of the CO vibrational spectrum assume top site adsorption as the peak intensity from the CO stretch appears at 
$2070 \mathrm{~cm}^{-1}$. Payne et al. [4] in their detailed analysis of the kinetic desorption data of CO on $\mathrm{Rh}(111)$ predicted rises and drops in the isosteric heat of adsorption signaling the existence of ordered phases. These occurred at coverages of $1 / 3$ and 2/3 ML (for the $(\sqrt{3} \times \sqrt{3}) \mathrm{R} 30^{\circ}$ ordering) due to nearest neighbor repulsion, and at $1 / 4$ and $3 / 4 \mathrm{ML}$ (corresponding to the two $2 \times 2$ phases) and $1 / 2 \mathrm{ML}$ (corresponding to the $2 \times 1$ order), due to next nearest neighbor interactions.

Concerning the adsorption site, deKoster et al. [5] used the extended Huckel method with corrections for electrostatic core repulsions to determine the most favorable sites of $\mathrm{CO}$ adsorbed on $\mathrm{Rh}(111)$. They concluded that this site is the 3-fold hcp hollow site, which appears to be contrary, at least in part, to several experimental findings. For example Koestner et al. [6] using LEED, found that $\mathrm{CO}$ adsorbs on the top site in the $(\sqrt{3} \times \sqrt{3}) \mathrm{R} 30^{\circ}$ structure. These assignments were also proposed by Beutler et al. [7] based on their high-resolution core level photoemission studies. A recent automated tensor LEED analysis by Gierer et al. [8] confirmed these assignments and also indicated a surface buckling where top site rhodium atoms are pulled out of the surface by $0.08 \AA$. Previous HREELS studies suggested that bridge sites were occupied by the $\mathrm{CO}$ molecules, indicating that vibrational spectroscopy cannot readily distinguish between adsorbate bonding at three-hold hollow and bridge sites. In the same work, Beutler et al. assigned the top site to $\mathrm{CO}$ in the lower coverage $(2 \times 2)$ structure as well, at $0.25 \mathrm{ML}$. As we shall see from our STM results, the question of the adsorption site is still unresolved as we found evidence of 3 -fold hollow occupation even at the lowest coverage $(2 \times 2)$ structure at $0.25 \mathrm{ML}$. In fact it appears that the adsorption site can be a strong function of the presence of other adsorbates, which might be present on purpose or as impurities. For 
example, $\mathrm{CO}$ always occupies the 3-fold hcp hollow site in the coadsorption studies of $\mathrm{CO}$ and benzene by Van Hove et al. $[10,11]$.

Other important parameters that can influence the structure of chemisorbed $\mathrm{CO}$ are the gas pressure and sample temperature used to achieve a given coverage. High coverages are often obtained by working at low temperatures, usually liquid nitrogen temperature. High coverage can also be achieved at room temperature by high pressure of the $\mathrm{CO}$ gas phase. We are then dealing with the question of whether the structures formed are in true thermodynamic equilibrium or are metastable ones. Although this is not important from the atomistic point of view, it is certainly relevant in catalytic environments. STM, being a real space technique, can illuminate many of the atomic scale details of the structure of adsorbed phases. The presence of defects and foreign adsorbates in small concentrations and in disordered arrangements can be ascertained readily while they escape detection with conventional, space averaging or diffraction based techniques.

\section{Experimental}

The experiments were carried out in a stainless steel ultra-high vacuum (UHV) chamber with a base pressure of $5 \times 10^{-10}$ torr. The chamber is equipped with LEED optics, Auger electronics, ion sputtering, mass spectrometer, and STM. 90\% Platinum $10 \%$ Rhodium tips were used. Unless otherwise noted, the images were taken with the sample positively biased at $100 \mathrm{mV}$ and a tunneling current of $1 \mathrm{nA}$.

The rhodium (111) orientation single crystal sample was cleaned by repeated cycles of argon ion sputtering $\left(5.0 \times 10^{-5}\right.$ torr of $\left.\mathrm{Ar}^{+}\right)$, followed by annealing in oxygen 
$\left(5.0 \times 10^{-8}\right.$ torr of $\left.\mathrm{O}_{2}\right)$ and in vacuum. Cleanliness of the sample was determined by Auger Electron Spectroscopy (AES), and the order of the surface structure was determined by the presence of the sharp diffraction beams in the LEED pattern of the clean surface. Before exposure, the sample was flashed to $700 \mathrm{~K}$ and allowed to cool to $300 \mathrm{~K}$. CO was introduced into the chamber and was kept at a constant background pressure. The magnitude of the pressure depended upon which $\mathrm{CO}$ adsorption structure was to be imaged. No annealing was carried out after exposure to CO. LEED patterns were recorded before imaging with the STM.

\section{Low coverage $(2 \times 2)$ structure}

After exposing the clean Rh surface to $\mathrm{CO}$, a (2×2) LEED pattern was obtained. During the experiments a constant background pressure of $\mathrm{CO}$ gas was maintained at $2 \times 10^{-9}$ torr. The STM images revealed the presence of steps characteristic of the miscut angle relative to the (111) plane. They separate terraces of 50 to $150 \AA$ average width. Numerous bright spots which we assign to unknown contamination are visible. Often they cause instabilities in the tunneling gap when the tip scans over them. In most of the surface a periodic $(2 \times 2)$ structure is observed with a corrugation that varies between 0.1 and $0.4 \AA$ A depending on tunneling conditions of bias and gap resistance and also on less controllable parameters such as the tip structure and composition. An example of such images is shown in Figure 5-1.

It is important to notice the symmetry of the unit cell in the higher resolution STM images. As can be seen in the figure, the two triangular halves of the diamond shaped cell are quite different as shown quantitatively in the profile along the only mirror 
symmetry plane of the structure, which is perpendicular to the surface and in the direction of the long diagonal (the $\sqrt{3}$ or [112] direction). The center of one of the triangles appears deep while the other appears shallow. While an asymmetry is to be expected, the magnitude of the difference in tunneling probability calls for a rather substantial difference in the electronic structure of the two sites that is sensed by the tip. For example, if CO sits on top sites, each half of the unit cell is centered on a 3-fold hollow site of the hcp and fcc types. Because it is only the second layer metal atom that distinguishes these two sites, we expect a small difference in STM tunneling probabilities when the tip is located above them. However, if $\mathrm{CO}$ sits on the 3 -fold hollow sites (either fcc or hcp), one half cell is centered on a first layer $\mathrm{Rh}$ atom, while the other is centered on a different hollow site. Such a geometry should result in substantial orbital overlap differences between the tip and the $\mathrm{CO}$ orbitals due to the interaction with the $\mathrm{Rh}$ atom. This appears to be evidence of a 3-fold binding site for $\mathrm{CO}$ at the $0.25 \mathrm{ML}$ coverage that produces the $(2 \times 2)$ structure.

Additional support for this assignment comes from the observed $(2 \times 2)$ structure formed by $\mathrm{CO}$ when coadsorbed with aromatic meta-xylene molecules. The STM images in Figure 5-2 show a small amount of meta-xylene mixed with $\mathrm{CO}$. The $\mathrm{CO}$ forms a $(2 \times 2)$ structure with the same unit cell asymmetry as found in the pure $\mathrm{CO}(2 \times 2)$ structure of Figure 5-1. The meta-xylene molecules in this image appear as the bright triangles. It is reasonable to extrapolate the previous LEED results obtained for the benzene-CO system (both with 1 and $2 \mathrm{CO}$ molecules per unit cell), to the present case, due to the chemical similarity between the two aromatic benzene type molecules. As 


\section{$\mathrm{P}(\mathrm{CO}) \sim 2 \times 10^{-9}$ torr}

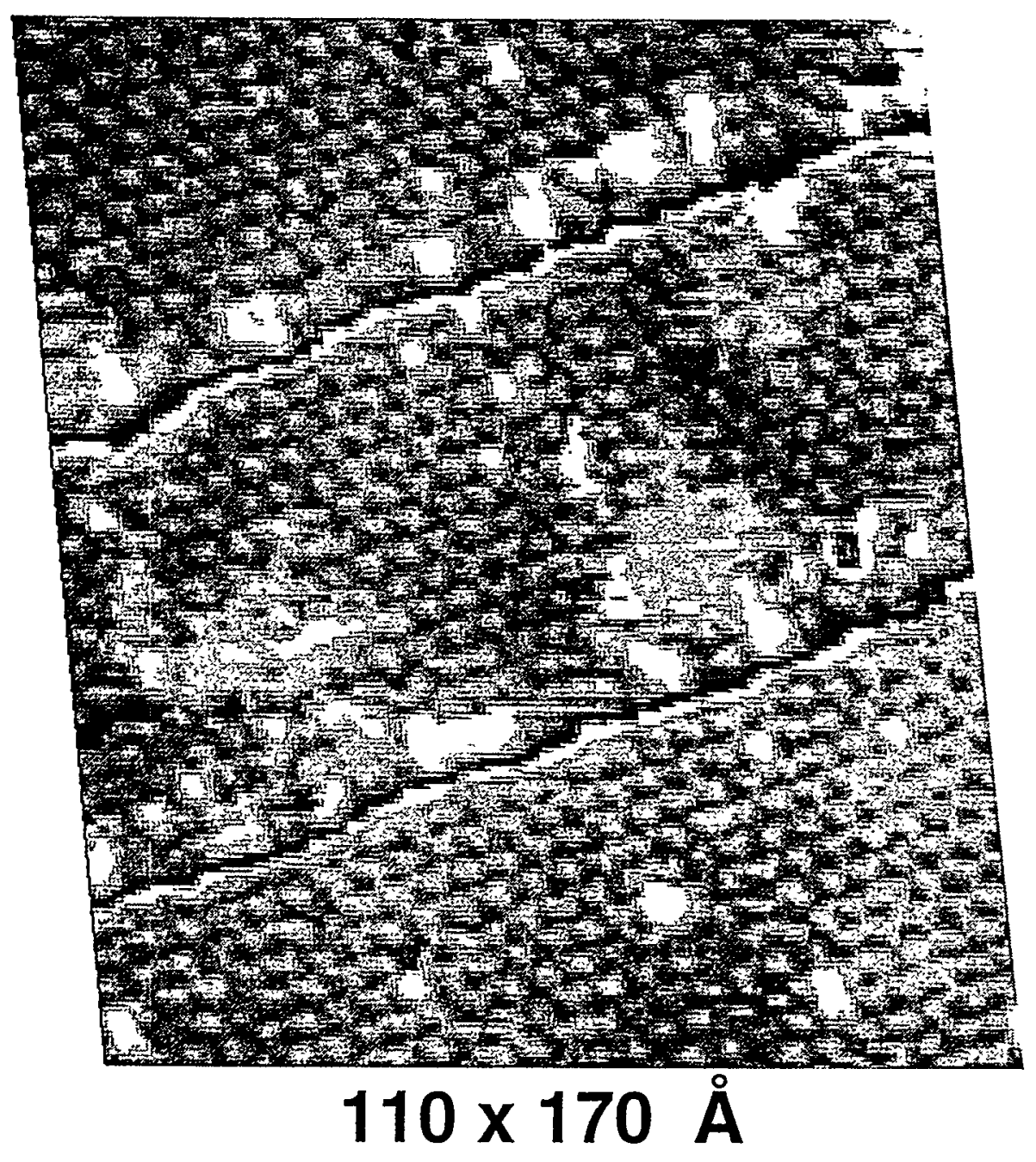

\section{CO- Corrugation $\Delta z=0.15 \AA$ $(\mathrm{V}=0.1 \mathrm{~V} ; \mathrm{I}=1 \mathrm{nA} ; \mathrm{R}=0.1 \mathrm{G} \Omega$ )}

Figure 5-1: A topographic image of the low coverage $(2 \times 2)$ structure formed by $\mathrm{CO}$ on the $\mathrm{Rh}$ (111) surface. There are three terraces in the image which have been flattened to increase the image quality. 

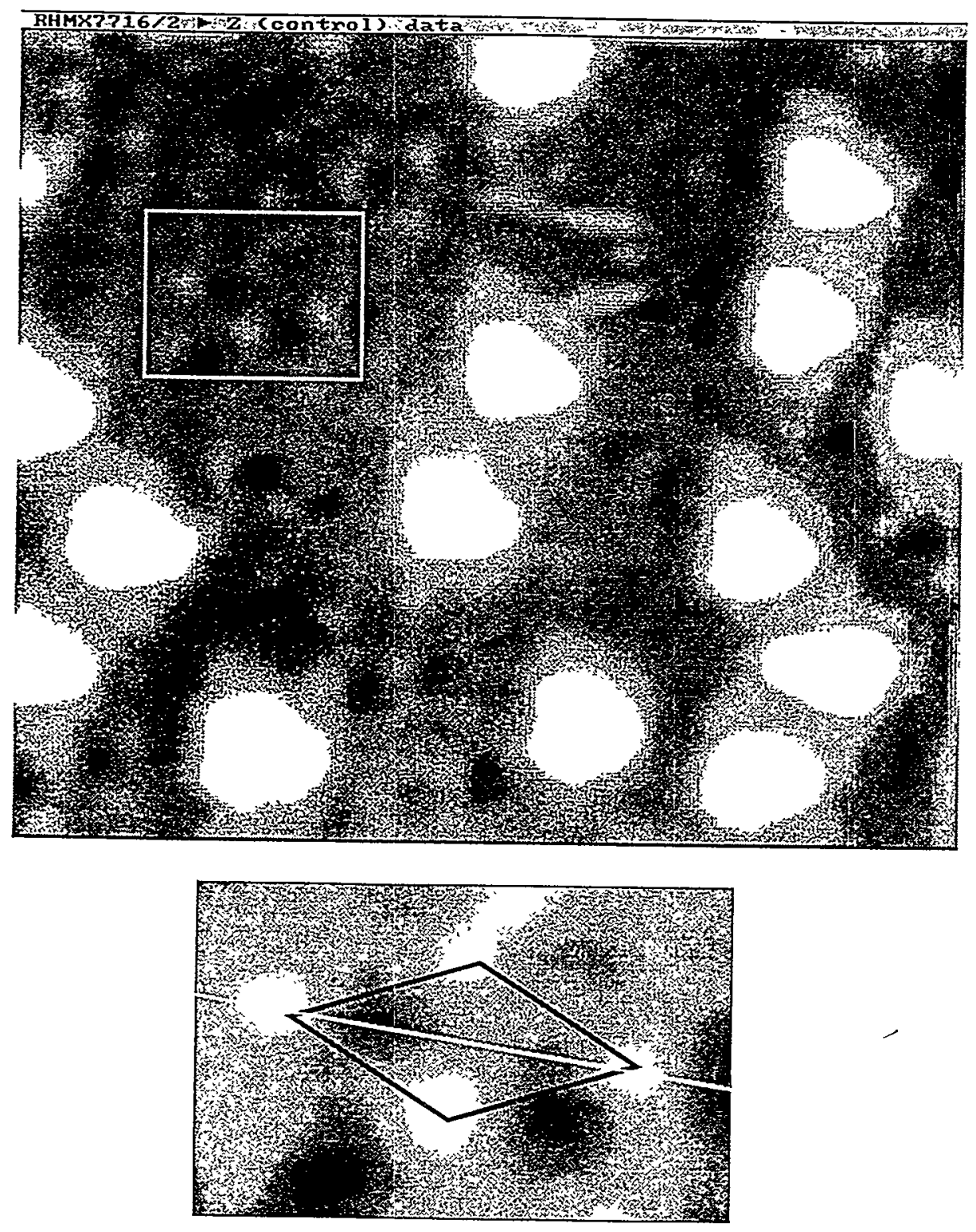

\section{$(2 \times 2)$ CO unit cell}

Figure 5-2: Top: A topographic image of the low coverage $(2 \times 2)$ structure formed by $\mathrm{CO}$ when co adsorbed with a small amount of meta-xylene. Bottom: A detail of the larger image with the asymmetry in the unit cell highlighted. 
with benzene, we expect meta-xylene to sit on hcp sites and $\mathrm{CO}$ to also sit on similar hcp sites.

\section{The $(2 \times 1)$ structure}

Exposing the Rh surface to $5 \times 10^{-8}$ torr of $\mathrm{CO}$, results in the formation of a LEED pattern of either $(2 \times 2)$ symmetry or to three domains of $(2 \times 1)$ symmetry. The STM images, obtained while maintaining the same background pressure of $\mathrm{CO}$, indicate without any doubt that it is the $(2 \times 1)$ structure that has formed. Domains in other directions were also observed. Images of the surface covered with this structure are shown in Figure 5-3. In the top image, a large scan area shows steps due to emerging dislocation planes crossing the surface. These steps are very straight and criss-cross each other at $60^{\circ}$ and $120^{\circ}$ angles as expected from compact (111)-type planes, which are the easy slippage planes. An example of the very characteristic triangular patterns formed is shown here. Since the steps are aligned along the compact [110]-type directions, they provide a very useful internal calibration of the crystal orientation for comparison with the atomic scale images. At higher magnification, the image shown below reveals the $2 \times 1$ periodicity with the unit cell sides clearly aligned along the compact rows of $\mathrm{Rh}$ atoms. The elongated shape of the maxima due to the $\mathrm{CO}$ molecules is along the long side of the unit cell. It is remarkable (and not understood at present) that the elongation is seen only along one of the two possible compact row directions.

\section{High coverage structures}

By exposing the Rh substrate to higher pressures of $\mathrm{CO}$ gas, above $10^{-7}$ torr, new structures were observed, that have never been reported before. At $10^{-6}$ torr, the 
periodicity formed by the $\mathrm{CO}$ overlayer is $(\sqrt{ } 7 \times \sqrt{ } 7) \mathrm{R} 19^{\circ}$. The determination was done by calibration of the angles between the unit cell sides of this structure with the steps of [110]-type orientation. As can be seen in Figure 5-4, this angle is $19^{\circ}$. Another support for the assignment is the observation of two domains rotated by $21^{\circ}$ relative to each other, as expected from this structure. These can be seen in the two large upper terraces of the figure, or coexisting in the same terrace at the bottom right of the figure. The corrugation of the CO structure is shown in the cursor profile to be of a few tenths of an Ångstrom. At higher resolution, as in the example of Figure 5-5, the unit cell structure is seen to consist of trimers of maxima separated by $2.5 \pm 0.4 \AA$, i.e., close to the Rh-Rh nearest neighbor distance of $2.69 \AA$. This corresponds most probably to three $\mathrm{CO}$ molecules sitting in three neighboring $\mathrm{Rh}$ sites in a triangular arrangement. Such a close $\mathrm{CO}-\mathrm{CO}$ separation also occurs in the dense $(2 \times 2)$ structure formed at $0.75 \mathrm{ML}$.

As the background $\mathrm{CO}$ pressure is increased, the periodicity of the $\mathrm{CO}$ structure remains $(\sqrt{ } 7 \times \sqrt{ } 7) \mathrm{R} 19^{\circ}$. However, new bright maxima appear inside the unit cell, in between trimers of $\mathrm{CO}$. The number of cells modified in this manner increases with $\mathrm{CO}$ pressure. In the example shown in Figure 5-5, obtained at about $10^{-5}$ torr, nearly 50\% of the $(\sqrt{ } 7 \times \sqrt{ } 7) \mathrm{R} 19^{\circ}$ cells contain the additional bright spot. We believe that the spots are due to additional $\mathrm{CO}$ molecules sitting on top sites. A schematic diagram of the structure is shown in the lower part of the figure. Thus, two types of $(\sqrt{ } 7 \times \sqrt{ } 7) \mathrm{R} 19^{\circ}$ structures exist that contain 3 and $4 \mathrm{CO}$ molecules. The coverage corresponding to each perfect structure would be 0.43 and $0.57 \mathrm{ML}$. We have marked examples of each type of cell with the letters $a$ and $b$ in the figure. 

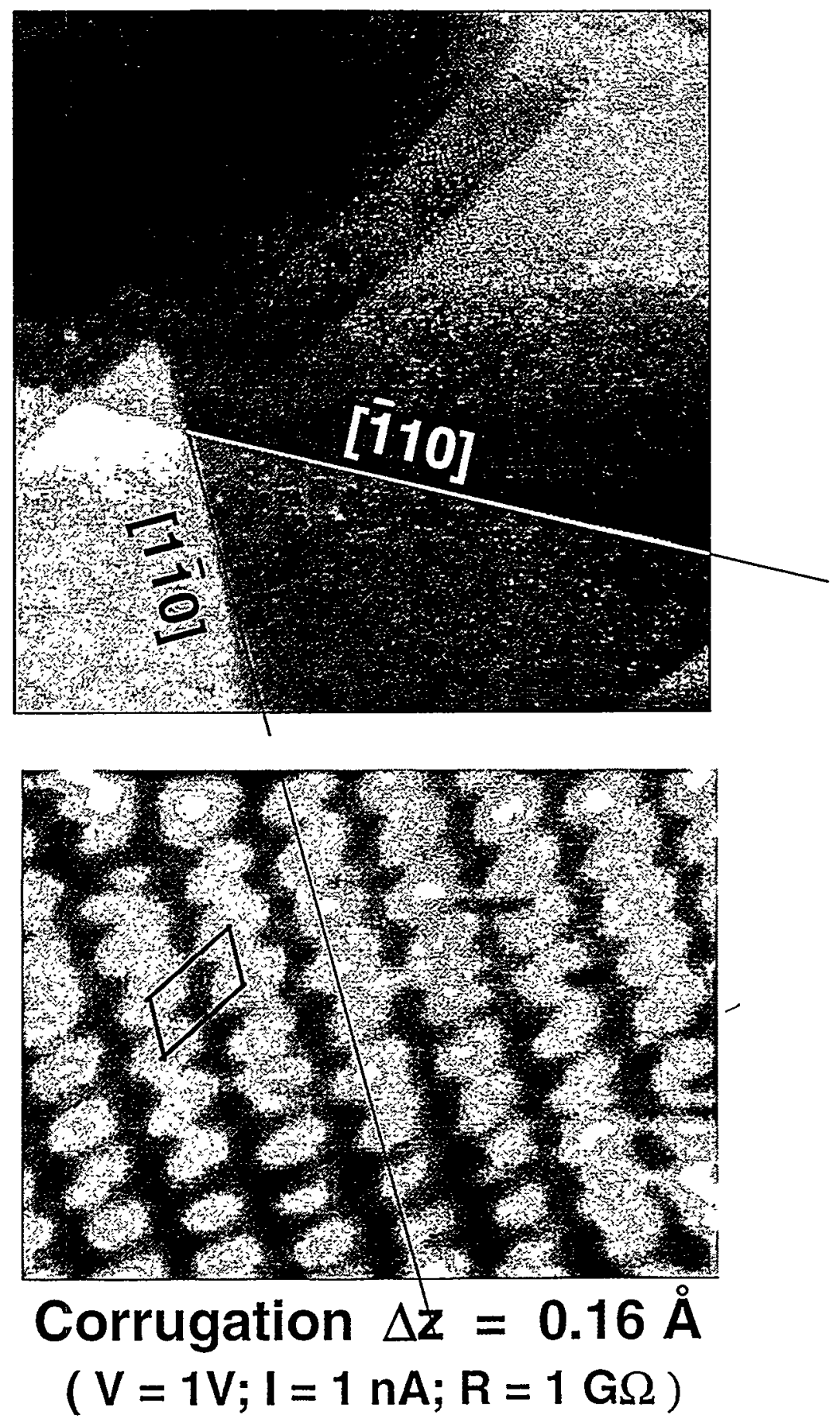

Figure 5-3: Topographic images of the (2x1) structure formed by CO on the Rh (111) surface (bottom). The orientation of the domains is confirmed by the geometry of the steps on the surface (top). 

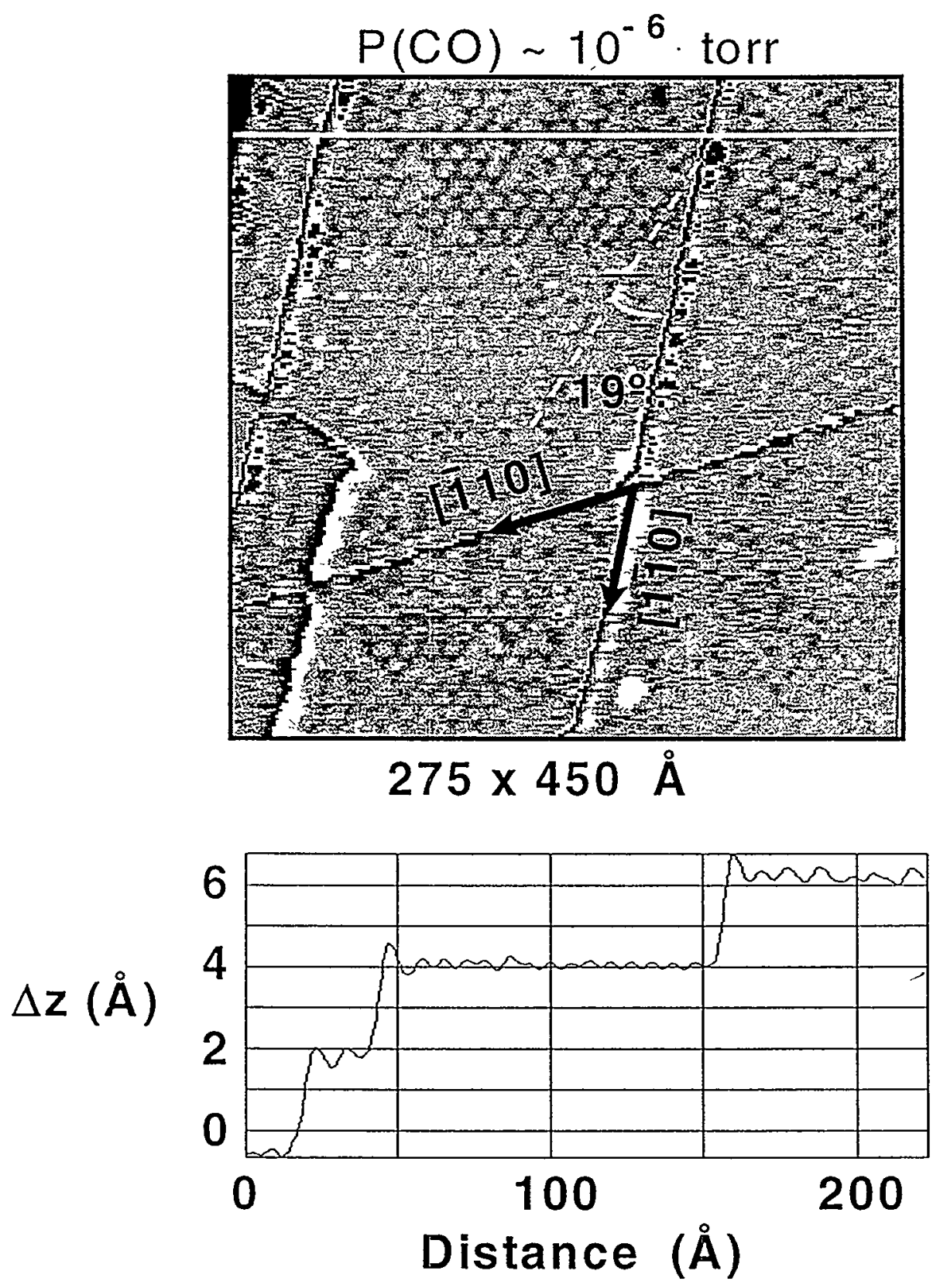

Figure 5-4: A topographic image of the $(\sqrt{ } 7 \times \sqrt{ } 7) \mathrm{R} 19^{\circ}$ structure formed by $\mathrm{CO}$ on the rhodium (111) surface, along with a line scan of the structure showing the corrugation and step heights. 


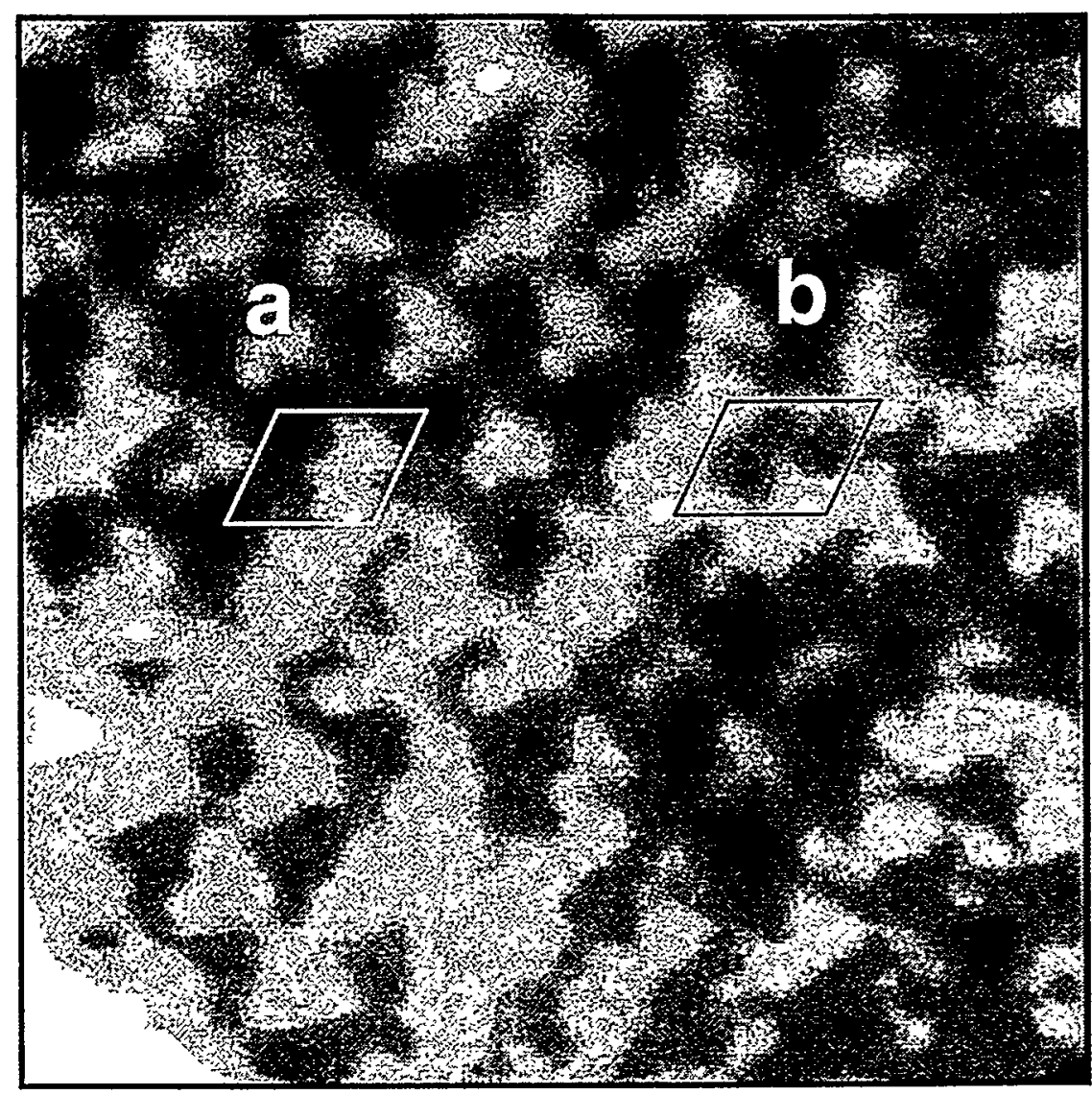

$$
\mathrm{P}(\mathrm{CO}) \sim 10^{-5} \text { torr }
$$

\section{Model: \\ $a=3 \mathrm{co} /$ unit cell \\ $b=4 \mathrm{CO} /$ unit cell}

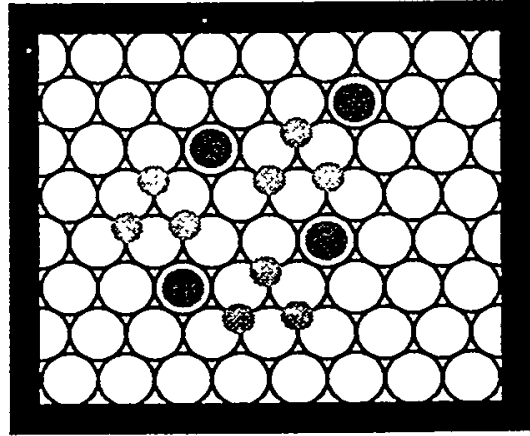

Figure 5-5: A topographic image of the $(\sqrt{ } 7 \times \sqrt{ } 7) \mathrm{R} 19^{\circ}$ structure. The unit cell with three CO's (a) and 4 CO's (b) are indicated. 


\section{Discussion}

Some of the surface science techniques probe ordered surface structures almost exclusively and at low pressures (LEED) while others are sensitive to surface bonding regardless of order (vibrational spectroscopy). LEED is not sensitive to small domains of adsorbates while assignments of adsorbate location from vibrational spectroscopy data can often be ambiguous. STM studies of adsorbate surface structure can be carried out over a 14 order of magnitude pressure range, the surface structure can be obtained in equilibrium with the gas phase and disordered as well as ordered phases are detectable. As a result we have been able to detect two new surface structures of $C O$ on $R h(111)$, the $(2 \times 1)$ and the $(\sqrt{ } 7 \times \sqrt{ } 7) R 19^{\circ}$ structures at coverages of 0.43 and 0.57 , respectively.

It is not clear from the experimental evidence whether that the top metal sites are occupied first by $\mathrm{CO}$ at the lowest coverages. There is evidence of surface reconstruction from reliable LEED crystalography studies. Reconstruction is also implicated by SFG studies at high CO pressures, however, there is no evidence for the formation of $\mathrm{Rh}(\mathrm{CO})_{2}$ carbonyls on $\mathrm{Rh}(111)$ that were detected by infrared spectroscopy over rhodium particles dispersed on alumina.

The existence of so many new CO chemisorbed structures on the $\mathrm{Rh}(111)$ surface is somewhat surprising, in view of the large number of studies in the past devoted to this system. The $(2 \times 1)$ structure, for example, was mentioned by Payne et al. as producing a rise and fall of the isosteric heat of adsorption [4]. According to the order-disorder transition theory of Walker et al. [13], the existence of $(2 \times 1)$ and/or $(\sqrt{3} \times \sqrt{3}) \mathrm{R} 30^{\circ}$ 
structures in hexagonal lattices, depends on the ratio $r$ of second to first nearest neighbor repulsion between $\mathrm{CO}$ molecules. A high value of $r(>0.2)$ tends to favor the existence of only $2 \times 2$ and $2 \times 1$ structures while a small value of $r(<0.2)$ favors the existence of $(\sqrt{3} \times \sqrt{3})$ R $30^{\circ}$ structures as well. It is possible that these interactions might be fine-tuned by small changes in the state of the substrate, for example the presence of impurities, the presence of subsurface atoms that might buckle the surface slightly, etc. It is know for example that such buckling, as produced by embedded $\mathrm{Ar}^{+}$atoms, can dramatically alter the chemisorption properties of Ru surfaces, by altering the electronic density between $\mathrm{Rh}$ atoms in the surface plane and below it. Since STM is a local probe, it permits the separate investigation of many structures that might exist in areas of the surface of different electronic structure or mechanical stress. Space averaging techniques on the other hand might conceivably miss the existence of many surface structures that might be important in catalysis, crystal growth, etc. Another important factor is that we performed our experiments always keeping a certain background pressure of $\mathrm{CO}$ gas, which implies that we are certainly closer to equilibrium conditions that in previous cases where $\mathrm{CO}$ is adsorbed, usually with the crystal at low temperature, and removed from the gas phase for analysis of the surface afterwards.

One finding of our work that should be stressed here concerns the adsorption site of $\mathrm{CO}$. Although the top site has been favored in most previous studies, particularly a low CO coverage ( $<0.33 \mathrm{ML}$ ), we find some evidence suggesting that, 3-fold hollow sites are preferentially occupied in the low coverage $(2 \times 2)$ structure. Such 3 -fold hollow site occupation was always thought to be less energetically favorable and only form at high coverage, as in the $(2 \times 2)$ structure at $0.75 \mathrm{ML}$, or when $\mathrm{CO}$ is coadsorbed with 
benzene. On the contrary we find the reverse trend: the top site is occupied, as in the $(\sqrt{ } 7 \times \sqrt{ } 7) \mathrm{R} 19^{\circ}$ structure, only when the coverage is above $0.5 \mathrm{ML}$.

The formation of trimers of $\mathrm{CO}$ molecules occupying a triangular set of nearest neighbor sites of the Rh surface in this structure deserves some comment. Here we find that again $\mathrm{CO}$ prefers the hollow sites, at least up to the coverage of $0.43 \mathrm{ML}$, where the $(\sqrt{ } 7 \times \sqrt{ } 7) \mathrm{R} 19^{\circ}$ structure with $3 \mathrm{CO}$ per unit cell is saturated. It is remarkable that even with many empty top sites available CO still prefers the hollow sites even at the cost of the repulsion of the nearest neighboring molecules. When, at higher pressures top sites are finally occupied in the $(\sqrt{ } 7 \times \sqrt{ } 7) \mathrm{R} 19^{\circ}$ structure, the top CO molecule appears very bright (i.e., with high contrast) in the STM images. This is a result of both electronic structure effects and also and more directly from the higher elevation of the molecule. A similar high contrast was observed in the case of the dense, $0.75 \mathrm{ML}$ coverage, $(2 \times 2)$ structure formed by $\mathrm{CO}$ on $\mathrm{Pd}(111)$ at low temperature [14]. 
References:

[1] J. T. Grant and T. W. Haas, Surf. Sci. 21 (1970) 76.

[2] P. A. Thiel, E. D. Williams, J. T. Yates, Jr. and W. H. Weinberg, Surf. Sci. 84 (1979) 54.

[3] L. H. Dubois and G. A. Somorjai, Surf. Sci., 91 (1980) 514.

[4] S. H. Payne, H. J. Kreuzer, K. A. Peterlinz, T. J. Curtis, C. Uebing and S. J. Sibener, Surf. Sci. 272 (1992) 102.

[5] A. deKoster and R. A. van Santen, J. Vac. Sci. Technol. A 6 (1988) 1128.

[6] R. J. Koestner, M. A. Van Hove and G. A. Somorjai, Surf. Sci. 107 (1981) 439.

[7] A. Beutler, E. Lundgren, R. Nyholm, J. N. Andersen, B. Setik and D. Heskett, Surf. Sci. 371 (1997) 381.

[8] M. Gierer, A. Barbieri, M. A. Van Hove and G. A. Somorjai, Surf. Sci. Submitted.

[9] M. A. Van Hove, R. F. Lin and G. A. Somorjai, J. Am. Chem. Soc. 108 (1986) 2532.

[10] M. A. Van Hove, R. F. Lin, G. S. Blackman and G. A. Somorjai, Acta Cryst. B43 (1987) 368.

[11] D. M. Zeglinski, D. F. Ogletree, T. P. Beebe, R. Q. Hwant, G. A. Somorjai and M. Salmeron, Rev. Sci. Inst. 61 (1990) 3769.

[12] J. A. Jensen, K. B Rider, Y. Chen, M. Salmeron and G. A. Somorjai, J. Vac. Sci. and Tech., submitted (1998) 
[13] J. S. Walker and M. Schick, Phys. Rev. B, 20 (1979) 2088.

[14] M. Rose, J. Dunphy, S. Behler, F. Ogletree and M. Salmeron, Surf. Sci., submitted (1998). 


\section{Chapter 6: Ethylidyne}

\section{Introduction}

The adsorption of hydrocarbons on the surface of metal catalysts has long been an important area of research. Hydrocarbons are produced and refined in large scale industrial processes from fuel production to polymer synthesis. Ethylene, $\mathrm{C}_{2} \mathrm{H}_{4}$ is one of the largest-volume commodity chemicals produced worldwide, with 1993 global production of 137 billion pounds (worth approx. $\$ 25$ Billion)[1]. Because of this there has been a significant amount of scientific work done which attempts to understand the chemistry of both the production of ethylene and ethylene itself. The study of the adsorption of ethylene on the Rh (111) surface showed the existence of an ordered $c(4 \times 2)$ structure on the surface[2]. It was soon recognized that the structure of the adsorbed ethylene included a change in the arrangement of the molecule into a $\mathrm{C}-\mathrm{CH}_{3}$ (ethylidyne) structure on the rhodium surface[3,4,5]. In addition, a low-temperature $(2 \times 2)$ structure was observed at $230-270 \mathrm{~K}$ [4]. It was recognized [6] that the ordered structure at room temperature was caused by the coadsorption of carbon monoxide with the ethylidyne. This coadsorbate induced ordering has been observed for a number of systems $[7,8,9]$, 
and is of great theoretical interest. There is no immediately obvious reason why the addition of a dissimilar molecule to a disordered monolayer should cause ordering of the molecules already on the surface. Schematic drawings of the two ethylidyne structures are shown in Figure 6-1

\section{Experimental}

The experiments were carried out in a UHV chamber with a base pressure of $5 \times 10^{-10}$ torr. The sample was cleaned by argon ion bombardment and cycles of heating in oxygen and heating in hydrogen, followed by a final heating in vacuum. Auger Electron spectroscopy was used to make sure the surface of the crystal was clean.

The ordered overlayers of ethylidyne and carbon monoxide were formed by first exposing the crystal to ethylene to form a saturated layer of ethylidyne on the surface.

The sample was then turned so that the surface was able to be monitored with the LEED apparatus. When the ethylidyne was alone on the surface, only the $1 \times 1$ substrate LEED pattern was evident. CO was then added to the chamber and the LEED pattern was monitored until the $\mathrm{c}(4 \times 2)$ pattern was formed. An example of the LEED patterns observed is shown in Figure 6-2. The CO gas exposure was then ended.

STM images were taken after the $c(4 \times 2)$ LEED pattern was observed. All STM images were taken in under UHV conditions at room temperature (298K). Unless otherwise noted, the images were taken at a bias voltage of $100 \mathrm{mV}$ and a current of $1 \mathrm{nA}$. 


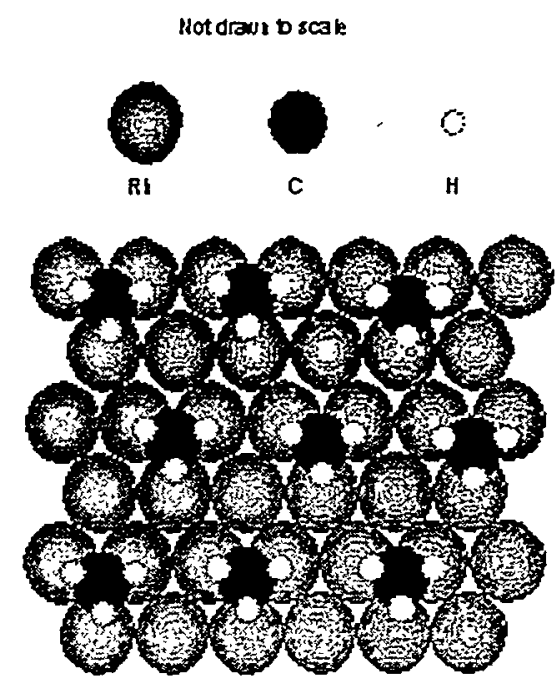

The $(2 \times 2)$ overlayer structure

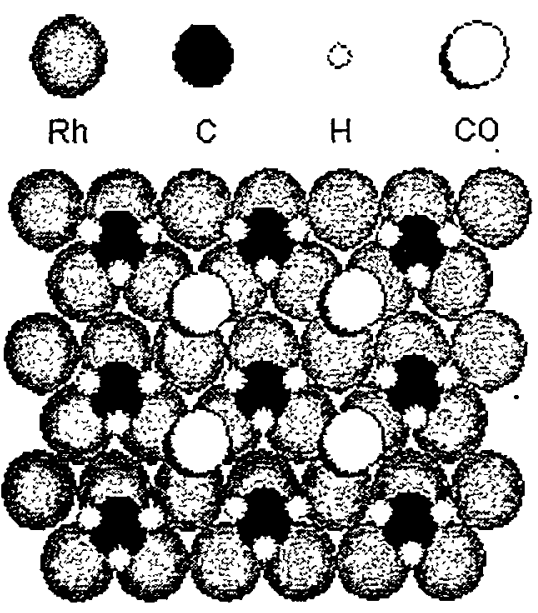

The $c(4 \times 2)$ overlayer structure

Figure 6-1: Drawing of the (2x2) overlayer formed by the adsorption of ethylidyne (top) and the $\mathrm{c}(4 \times 2)$ overlayer formed by the coadsorption of $\mathrm{CO}$ and ethylidyne(bottom). 

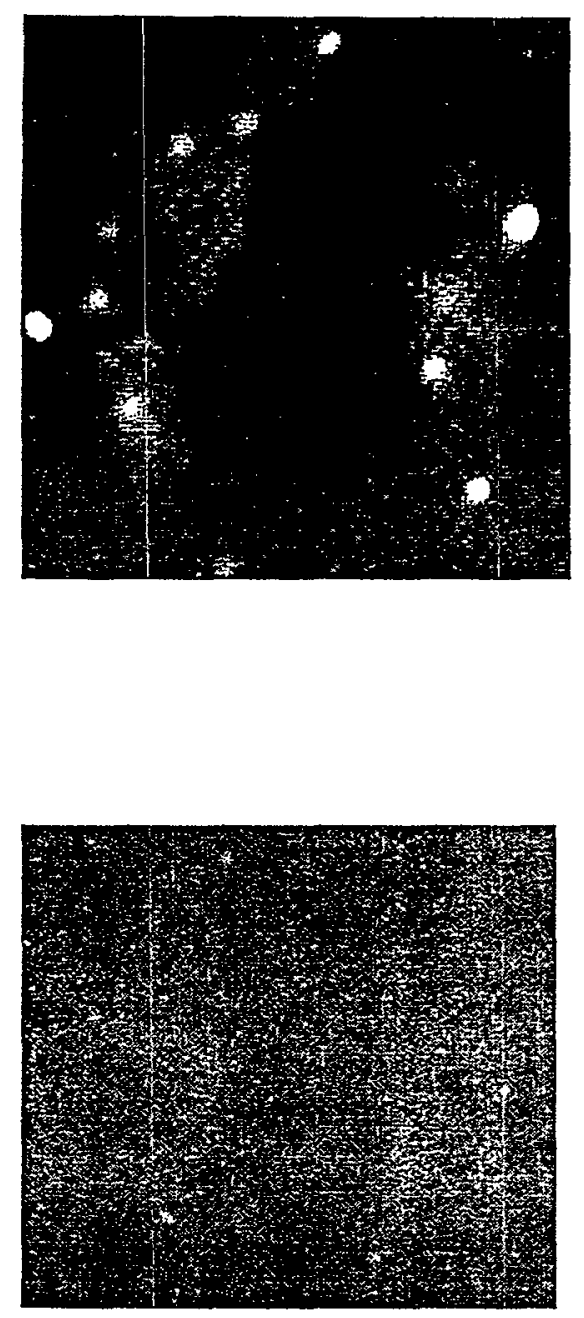

Figure 6-2: Top: The LEED pattern for the $c(4 \times 2)$ structure of $\mathrm{CO}$ coadsorbed with $\mathrm{CO}$ (some spots are obscured by the sample holder). Bottom: A LEED pattern of the clean surface. One of the $(1 \times 1)$ spots is obscured by the sample holder. 


\section{Results}

STM images of the Ethylidyne + CO system which are typical of those observed in these experiments are shown in Figure 6-3. In these images, only one distinct feature is observed, rather than one type of feature for the $\mathrm{CO}$ and one type for the ethylidyne. This feature has a relatively large corrugation of $.4 \AA$. A slice of the image showing this corrugation is shown in Figure 6-4. Outside of the ordered portions of the adsorbed layer, there are no features which would correspond to disordered ethylidyne. In some images, such as those shown in Figure 6-5, two different domains are observed. This is due to the rotation of the $\mathrm{c}(4 \times 2)$ structure with respect to the close-packed directions of the $\mathrm{Rh}$ atoms of the surface. An schematic diagram of this is also shown in Figure 6-5. The observation of these two domains which are related by a rotation by 120 degrees is another indication that we are really observing the $c(4 \times 2)$ pattern on the surface.

\section{Discussion}

The most interesting aspect of the STM images of CO coadsorbed with ethylidyne is the observation of only one kind of feature in the ordered domains. The structure of the ordered layer determined by LEED shows that the $\mathrm{CO}$ is interspersed with the ethylidyne, so that two features, an ethylidyne feature and a $\mathrm{CO}$ feature should be visible in the images. We must then determine which molecule is visible in the domain, and why the other is not.

Looking at the two components which are important in determining the tunneling from a molecule, physical height (the proximity of the tunneling electrons to the tip) and the electronic state (whether the overlap of the wavefunctions of the tip and sample is 


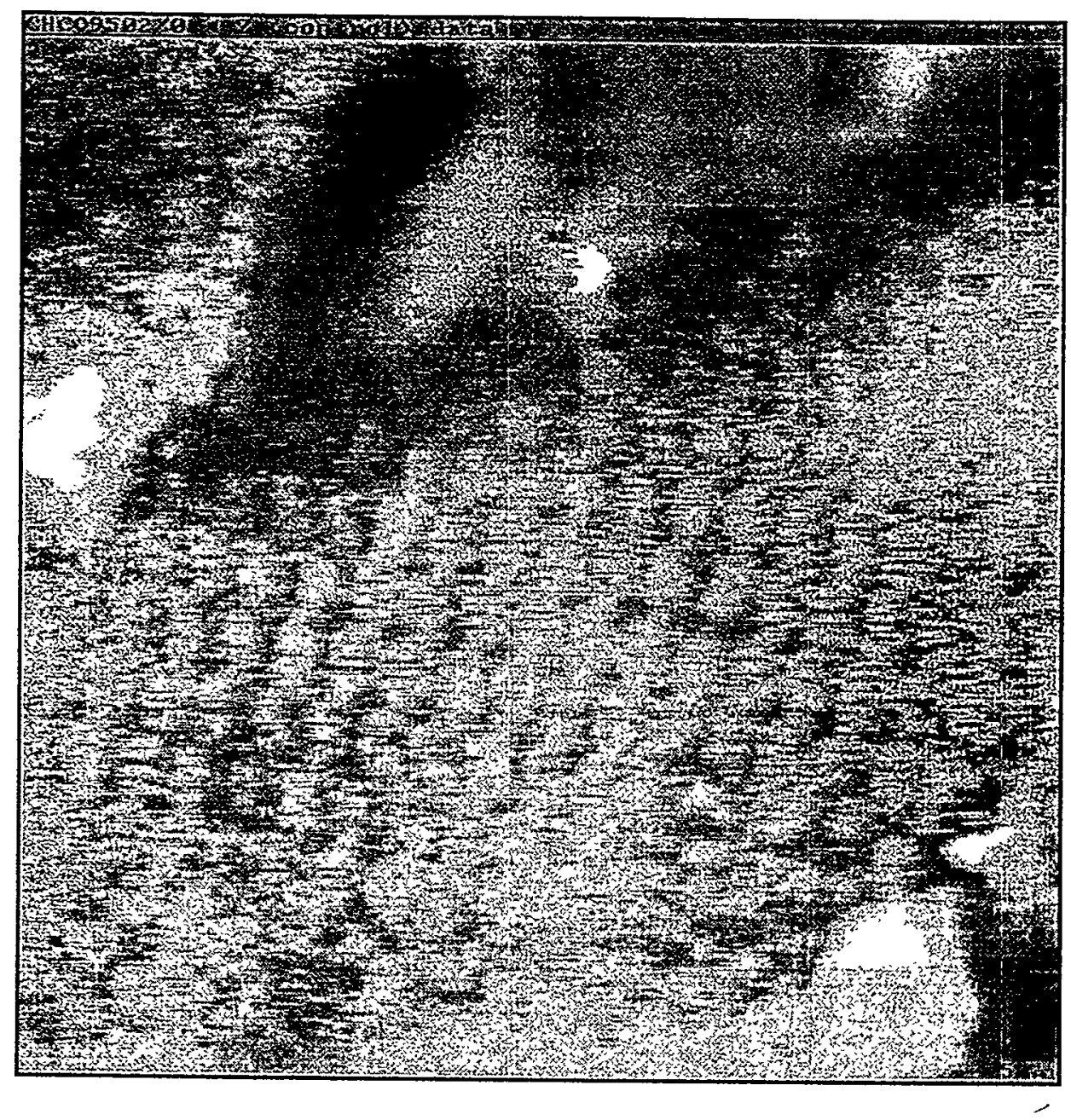

Figure 6-3: A topographic image of ethylidyne coadsorbed with CO on the rhodium (111) surface. 

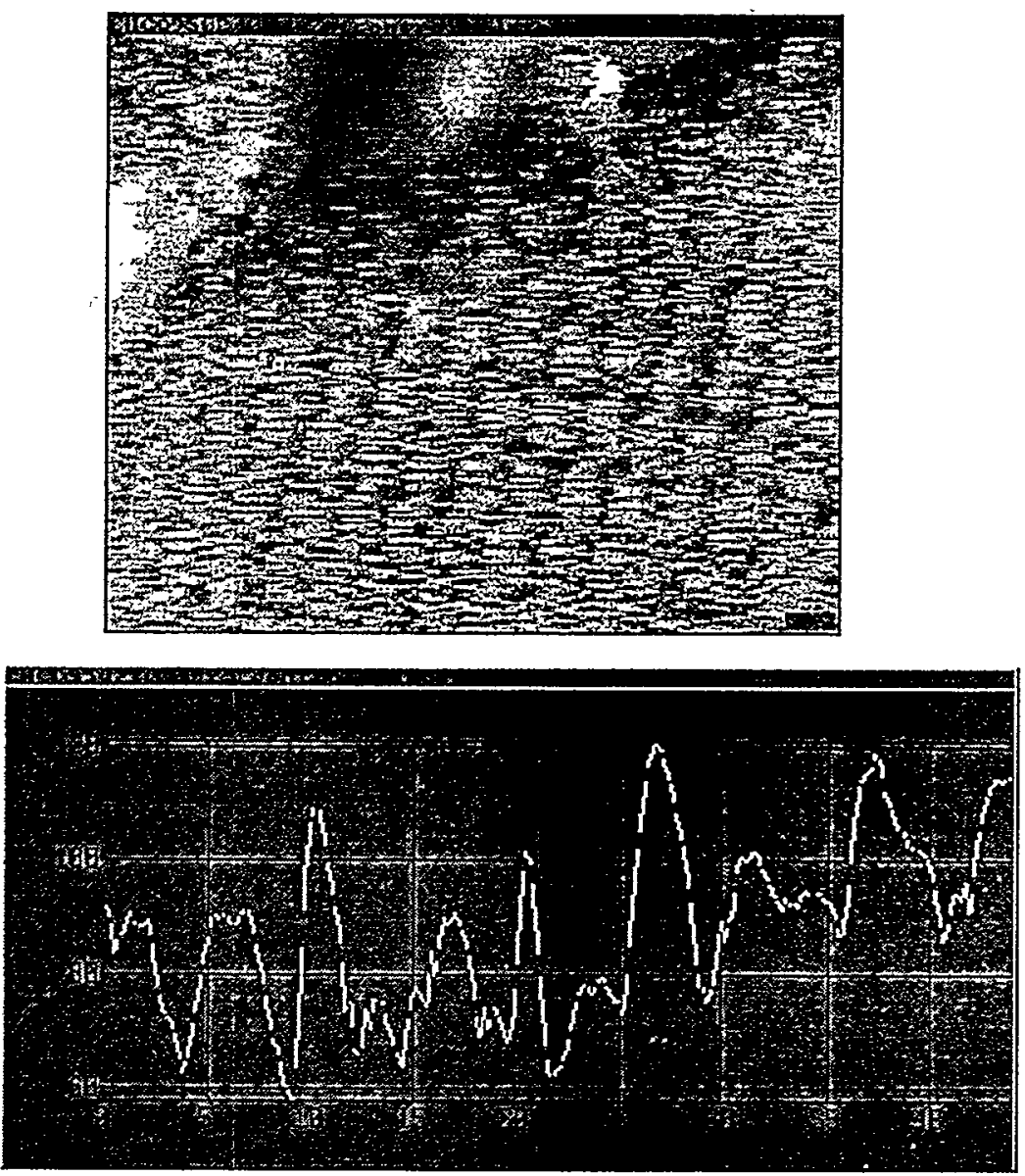

Figure 6-4: A slice through the STM image showing the corrugation of the ordered area. The units on the vertical axis are in picometers $(100 \mathrm{pm}=\AA)$. 

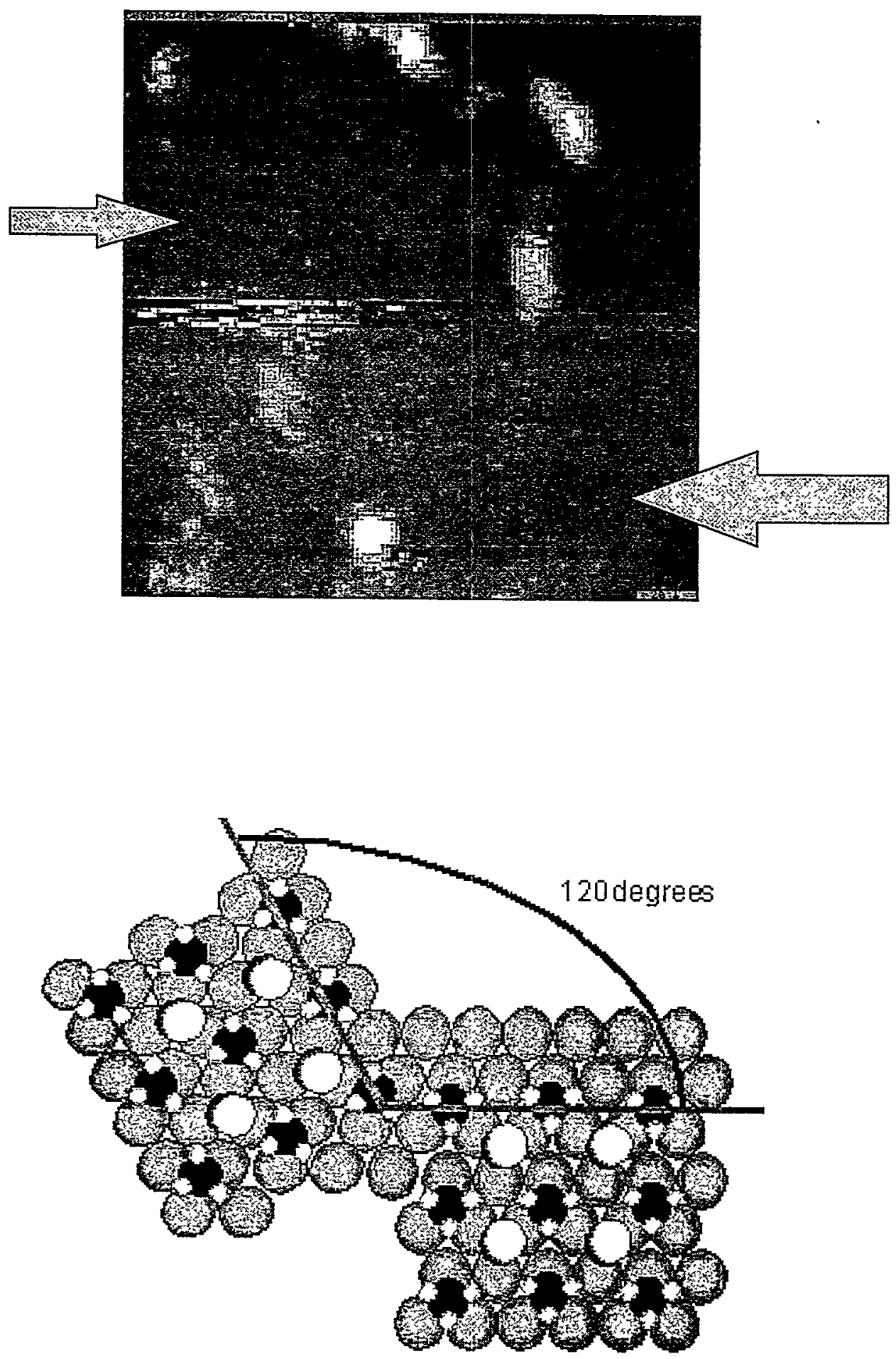

Figure 6-5: Top: A topographic image of $\mathrm{CO}$ coadsorbed with ethylidyne on the surface.

The two different domains are indicated by arrows. Bottom: A drawing of the two rotated domains. 
large) will help us determine what is happening at the surface. The LEED data[6] is able to give precise bond distances, which are shown in Figure 6-6, and these are what we would consider when looking at the physical height of the molecules on the rhodium surface. The ethylidyne has a carbon $2.90 \AA$ from the rhodium atoms on the surface, while the oxygen of the $\mathrm{CO}$ is $2.48 \AA$ from the surface rhodium atoms. While the hydrogen atoms in the ethylidyne would increase the height of the molecule on the surface, the tunneling from hydrogen will be very low, since it only has one electron compared with the twelve (four if only the valence electrons are considered) in carbon. This height difference of $0.42 \AA$ should cause a small difference in the tunneling current, but not by more than a factor of about $\mathrm{e}^{.94}=2.5$ (if the WKB approximation is used to estimate the tunneling current effect of distance). It is not clear that this difference would cause the $\mathrm{CO}$ to be effectively invisible on the surface.

Looking at the previous work on $\mathrm{CO}$ (see Chapter 5), the corrugation of $\mathrm{CO}$ on the surface is very small, usually $0.1-0.2 \AA$, while the features seen in the STM images have a much larger corrugation, about $.4 \AA$. This indicates that the features seen on the surface are more likely to be ethylidyne that $\mathrm{CO}$ due to the difference in the corrugation of the molecules when observed on the $\mathrm{Rh}$ surface.

While some of the corrugation difference should be due to the difference of the van der Waals heights of the molecules, the other effect is the relative energies of the states which are involved in the tunneling process. The tunneling process is, to a good approximation an elastic one, so that the energy of the filled state from which 


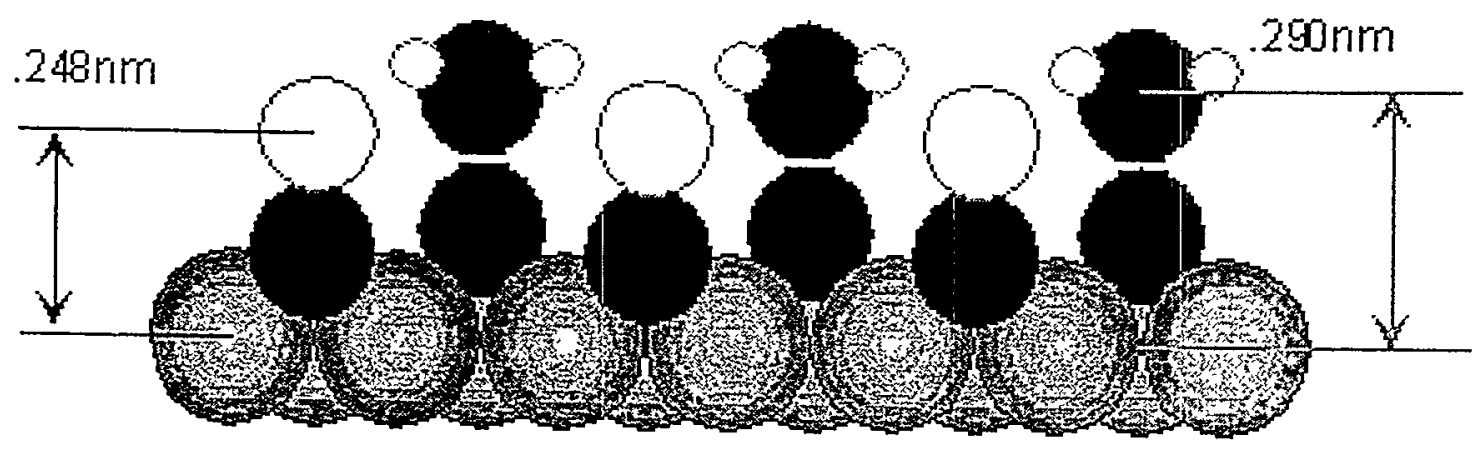

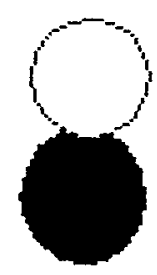

Co

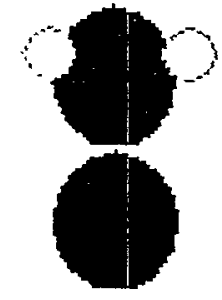

Ethylidyne

Figure 6-6: A diagram of the surface structure of the coadsorbed overlayer based on LEED data [8] 
the electron is tunneling is the same as the empty state into which it tunnels. If there are no empty states with the same energy, more complex processes must occur and the tunneling current is decreased. In the case of $\mathrm{CO}$, the low corrugation is attributable to the low lying HOMO (Highest Occupied Molecular Orbital) usually designated $5 \sigma$. This orbital has been observed to be at an energy that is approximately $2 \mathrm{eV}$ below the rhodium fermi level [11]. Since the bias on the sample is generally .leV, this means that the filled stated of $\mathrm{CO}$ are still about $2 \mathrm{eV}$ lower in energy than the empty states into which they are tunneling. This energy difference would decrease the tunneling current significantly and would result in the lower corrugation of $\mathrm{CO}$ which is observed in these experiments.

This argument has been made for the benzene $+\mathrm{CO}$ system with the tip negatively biased rather than the sample (as in the ethylidyne + CO experiments).

In this case, both the energetic and distance effects would imply that the molecules which are visible on the surface is ethylidyne, not $\mathrm{CO}$. 
References

[1] SRI Industries, "Ethylene", Chemical Industries Newsletter, May/June 1995, <http://www-cmrc.sri.com/CIN/MayJune95/Article01.html>, May 17, 1999.

[2] D.G. Castner, B.A. Sexton, and G. A. Somorjai, Surf. Sci. 71 (1978) 519.

[3] L.L. Kesmodel, L.H. Dubois, and G.A. Somorjai, J.Chem. Phys. 70 (1979) 2180.

[4] L.H. Dubois, D.G. Castner, and G. A. Somorjai, J. Chem. Phys. 72 (1980) 5234.

[5] R.J. Koestner, M.A. Van Hove, and G.A. Somorjai, Surf. Sci. 121 (1982) 321.

[6] G.S. Blackman, C.T. Kao, B.E. Bent, C.M. Mate, M.A. Van Hove, and G.A. Somorjai, Surf. Sci. 207 (1988) 66.

[7] C.M. Mate, B.E. Bent, and G.A. Somorjai, J. Electron Spectrosc. Rel. Phenom. 39 (1986) 205.

[8] R.F. Lin, G.S. Blackman, M.A. Van Hove, and G.A. Somorjai, Acta Cryst. B 43 (1987) 368 .

[9] H. Ohtani, M.A. Van Hove, and G.A. Somorjai, J. Am. Chem. Soc. 92 (1986) 3974.

[10] H. Ohtani, R.J. Wilson, S. Chiang, and C. M. Mate, Phys. Rev. Lett. 60 (1988) 2398.

[11] E. Bertel, G. Rosina, and F.P. Netzer, Surf. Sci. 172 (1986) 172. 


\section{Chapter 7: Cyclohexane Derivatives}

\section{Introduction}

Reactions involving cyclic molecules are representative of an important subset of hydrocarbon chemistry. These chemicals are important in the formulation and refining of fuels as well as in other areas of chemistry. Among cyclic molecules, cyclohexane has particular importance since one of the products of dehydrogenation is benzene, an interesting molecule both in practical and theoretical terms because of its aromaticity. The catalytic dehydrogenation reactions of cyclohexane to cyclohexene and 1,3- and 1,4cyclohexadiene have been studied on a number of metal surfaces, including palladium [1], platinum [2,3], copper [4], and ruthenium [5]. (Schematic drawings of the structures of these cyclohenane derivatives are shown in Figure 7-1.) Rhodium, however, has only very recently been a subject of systematic investigation for cyclohexane catalysis. In addition, past research on cyclohexane derivatives has focused on the use of vibrational spectroscopy, particularly High Resolution Electron Energy Loss Spectroscopy (HREELS). The lack of information in the literature on cyclohexane chemistry on the rhodium surface needs to be addressed. STM experiments can be used 


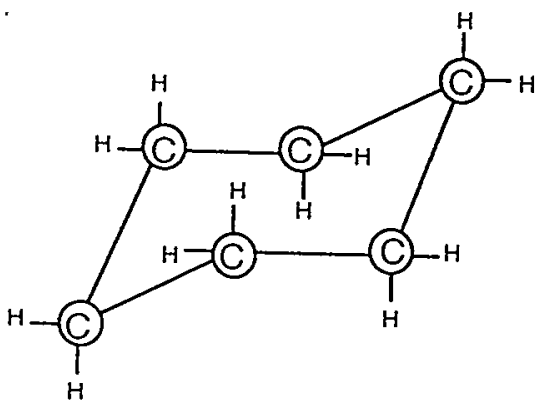

Cyclohexane

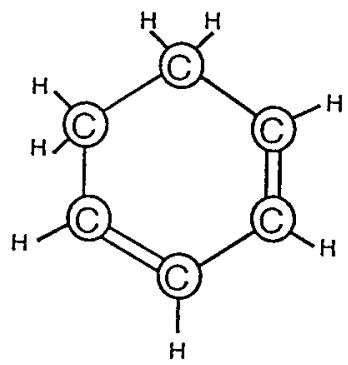

1,3-Cyclohexadiene

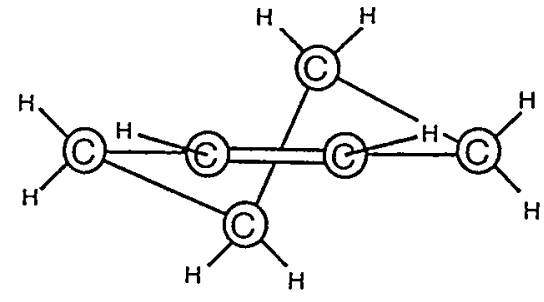

Cyclohexene<smiles>c1ccccc1</smiles>

Benzene<smiles></smiles>

1,4-Cyclohexadiene 
to determine the structure of the adsorbed molecules. Another open question is whether or not the cyclohexane adsorbed on the surface at room temperature remains intact or whether it undergoes dehydrogenation to cyclohexene, cyclohexadiene, or benzene. Using the information about surface structure gained through the STM experiments, as well as information on the symmetry of the molecules, the chemical identity of the molecules on the surface can be surmised.

\section{Experimental}

Cyclohexane, cyclohexene and 1-4 cyclohexadiene were obtained from Acros chemicals and was $>99 \%$ pure.

The experiments were carried out in a stainless steel ultra-high vacuum (UHV) chamber with a base pressure of $5 \times 10^{-10}$ torr. The chamber is equipped with LEED optics, Auger electronics, ion sputtering, mass spectrometer, and STM. Images were taken at room temperature in topographic mode. The $x$ - and $y$-piezoelectric displacements were calibrated with atomically resolved images of Rhodium; an example is shown in Figure 7-2.

The rhodium (111) single crystal sample was cleaned by repeated cycles of argon

ion sputtering ( $5.0 \times 10^{-5}$ torr of Ar), followed by annealing in oxygen ( $5.0 \times 10^{-8}$ torr of O) and vacuum. Cleanliness of the sample was determined by Auger Electron Spectroscopy (AES), and the order of the surface structure was determined by the presence of the sharp diffraction beams in the LEED pattern of the clean surface. Before exposure, the sample was flashed to $700 \mathrm{~K}$ and allowed to cool to $300 \mathrm{~K}$. For each molecule the vapor was dosed onto the surface of the clean rhodium surface though a 

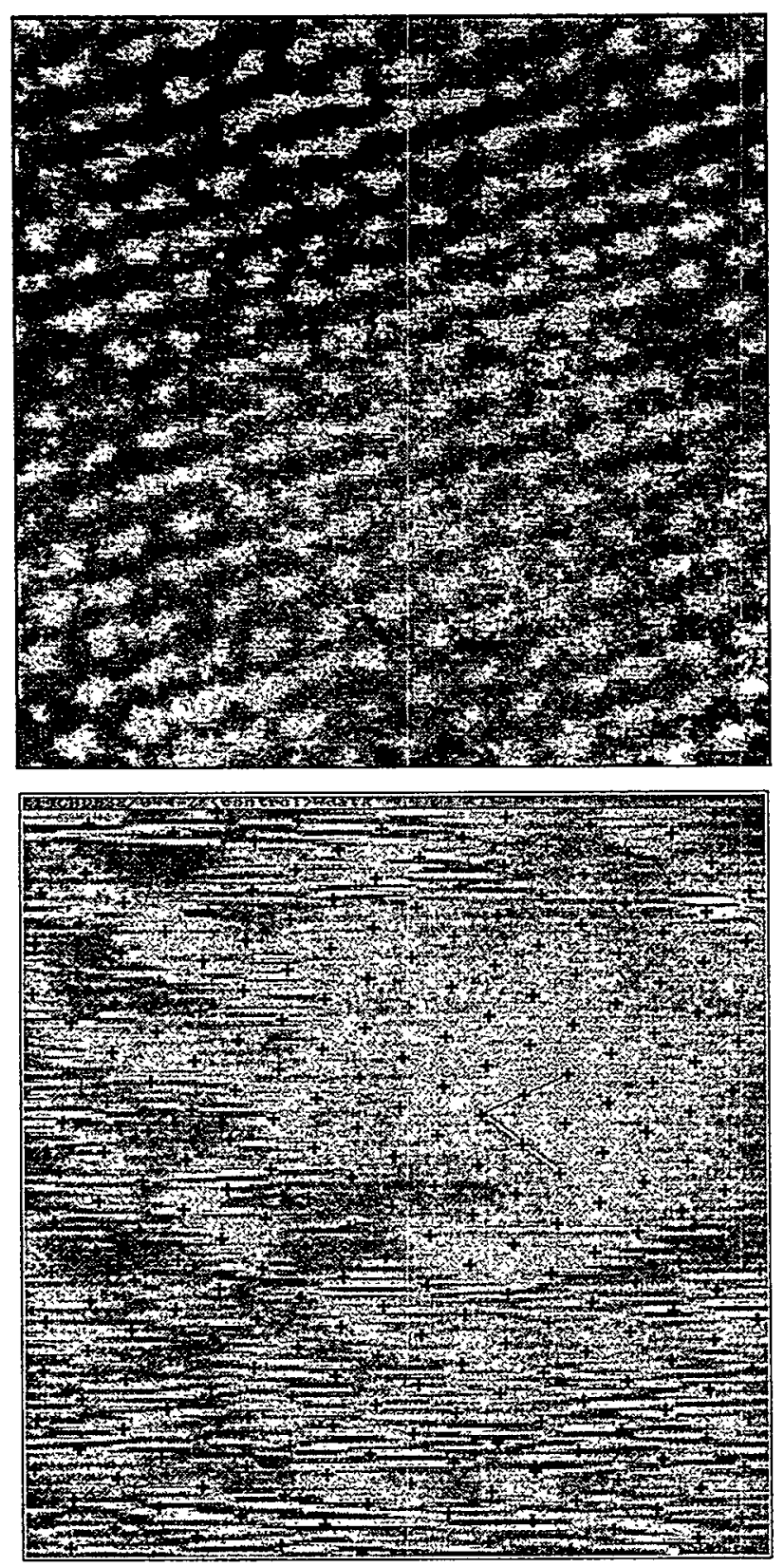

Figure 7-2: Top: An example of the atomically resolved rhodium metal surface. Bottom:

The calibration of distances is achieved by matching the distance between the rhodium atoms to the distance between grid points. 
leak valve with the aperture within $1 \mathrm{~cm}$ of the rhodium surface. LEED patterns were recorded prior to moving the sample to the STM stage.

\section{Cyclohexane Adsorption}

For the cyclohexane $\left(\mathrm{C}_{6} \mathrm{H}_{12}\right)$ experiments, $2 \mathrm{~L}$ were dosed onto the surface. LEED patterns showed no significant spots except the $(1 \times 1)$ spots of the $\mathrm{Rh}(111)$ surface. STM images of the surface were taken, but no images contained features which could be ascribed to the adsorption of cyclohexane. Since this might be due to the diffusion of the molecules on the surface, the addition of $\mathrm{CO}$ as a background gas was used, since $\mathrm{CO}$ has shown the ability to stop the diffusion of cyclic molecules on the surface of rhodium (see Chapter 5). Images were obtained of the $\mathrm{CO}$ on the surface, but again, no features which could be interpreted as adsorbed cyclohexane were observed.

\section{Cyclohexene Adsorption}

Experiments on the adsorption of cyclohexene on the rhodium surface used a range of exposures. Exposures below 3L resulted in only a diffuse background (aside from the (1x1) rhodium spots) in the LEED pattern taken before STM imaging. STM images obtained at these low exposures are shown in Figure 7-3. These images show only very small domains of ordered molecules on the surface. They also have features which indicate the diffusion of molecules on the surface.

In order to try to reduce the mobility of the molecules on the surface, the exposure amounts were increased. At exposures of $15 \mathrm{~L}$, the LEED pattern shows a $3 \times 3$ structure. 


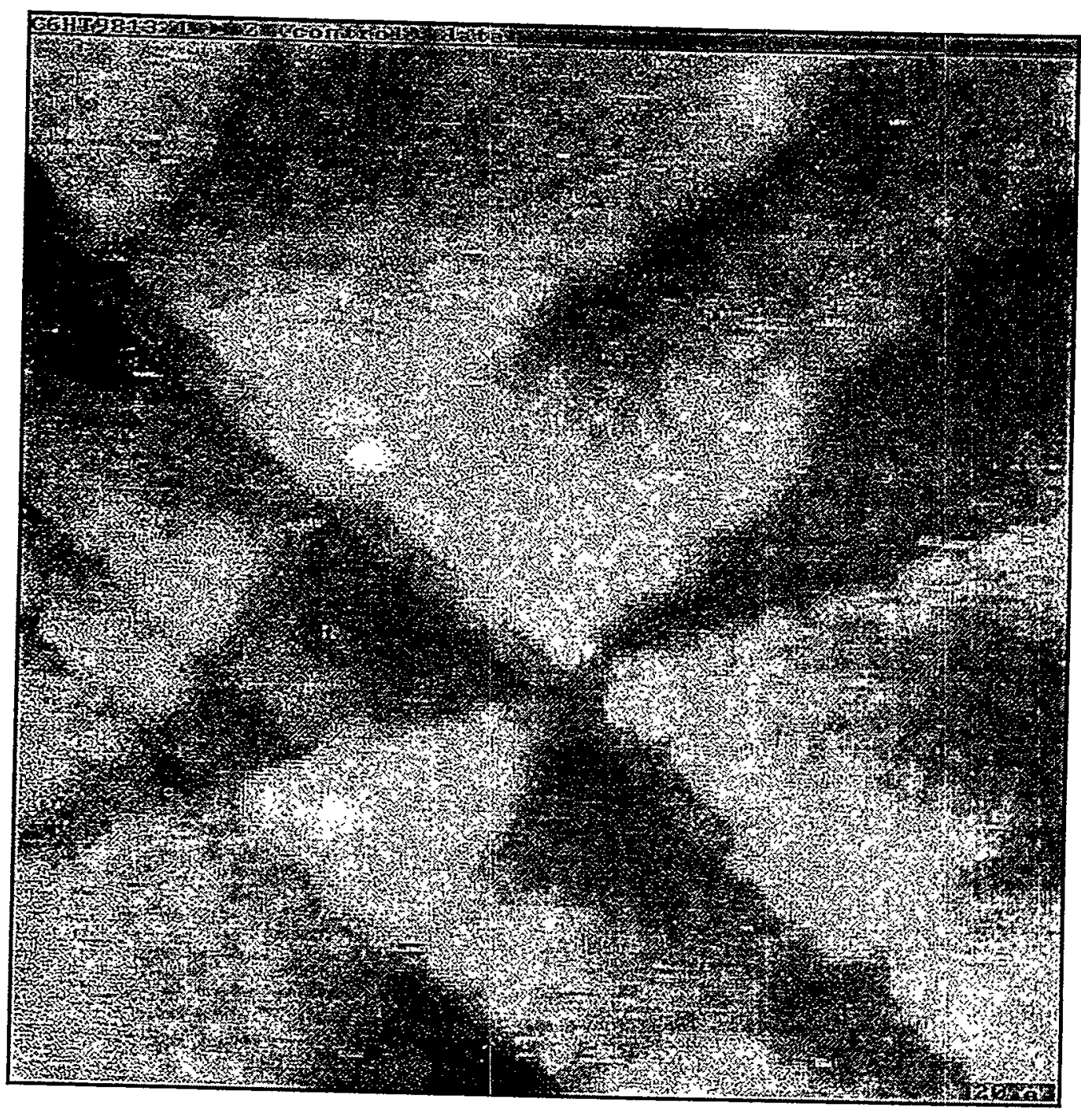

Figure 7-3: A topographic image of a low-exposure cyclohexene monolayer. While small patches of order are present, most of the surface is disordered. 
The STM images show large domains of cyclic' molecules on the surface. An example of these domains is shown in Figure 7-4. The molecules in the images seem to have internal symmetry. If these molecules were cyclohexene, the pi bond on the carbon ring would probably cause the imaged of the molecules to have a different tunneling current from the pi bond than from the rest of the ring, resulting in an asymmetry of the molecules.

Some experiments at the high exposures resulted in a adsorbed layer with a different structure. This is shown in Figure 7-5. It is not the (3x3) structure since the molecules in that structure are aligned in the close-packed direction, so there should be not rotation of the overlayer with respect to the unit cell of the surface $\mathrm{Rh}$ atoms. In Figure 7-5, the rotation of the structure is clearly shown by the light and dark lines. This angle is $19^{\circ}$. The unit cell, superimposed on the image in Figure 7-6, shows that the length of the unit cell is $12 \AA \times 14.5 \AA$. Again, the molecules which are seen in these images do not have any noticeable internal symmetry. An image taken atlow bias voltage $(25 \mathrm{mV})$ illustrates the ringed nature of the imaged molecules (Figure 7-7). Also, the ordered domains do not completely cover the surface. In the areas where there is no ordered domains, some individual molecules can be imaged, but most of this area is covered in "noise".

\section{1,4-Cyclohexadiene Adsorption}

For 1,4-cyclohexadiene adsorption, the rhodium surface was cleaned and then exposed to $2 \mathrm{~L}$ of the hydrocarbon. The adsorbed layer was checked for ordering with 


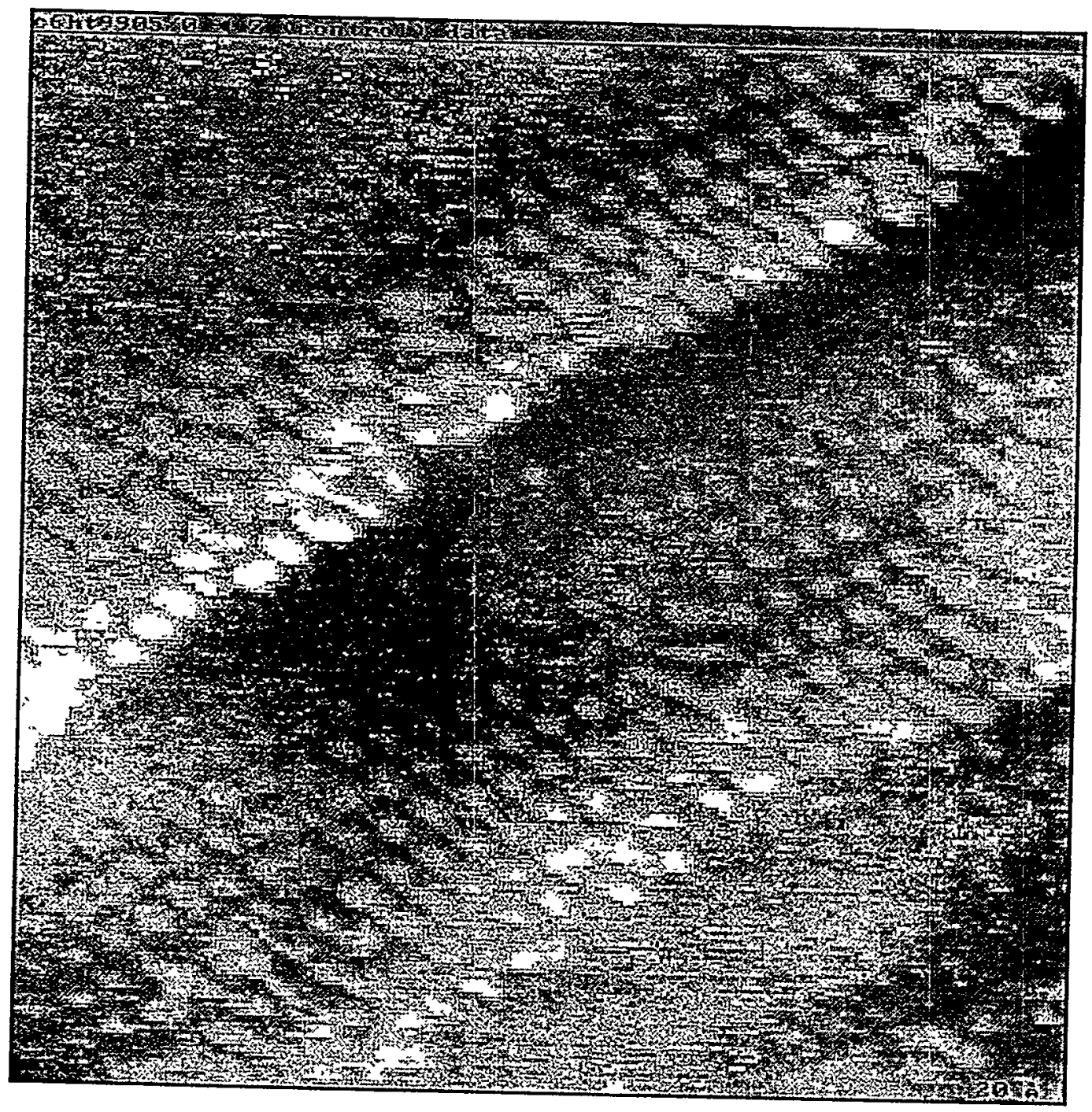

Figure 7-4: A topographic image of the layer formed by the adsorption of cyclohexane onto the rhodium surface. The symmetry of the overlayer is $(3 \times 3)$. 


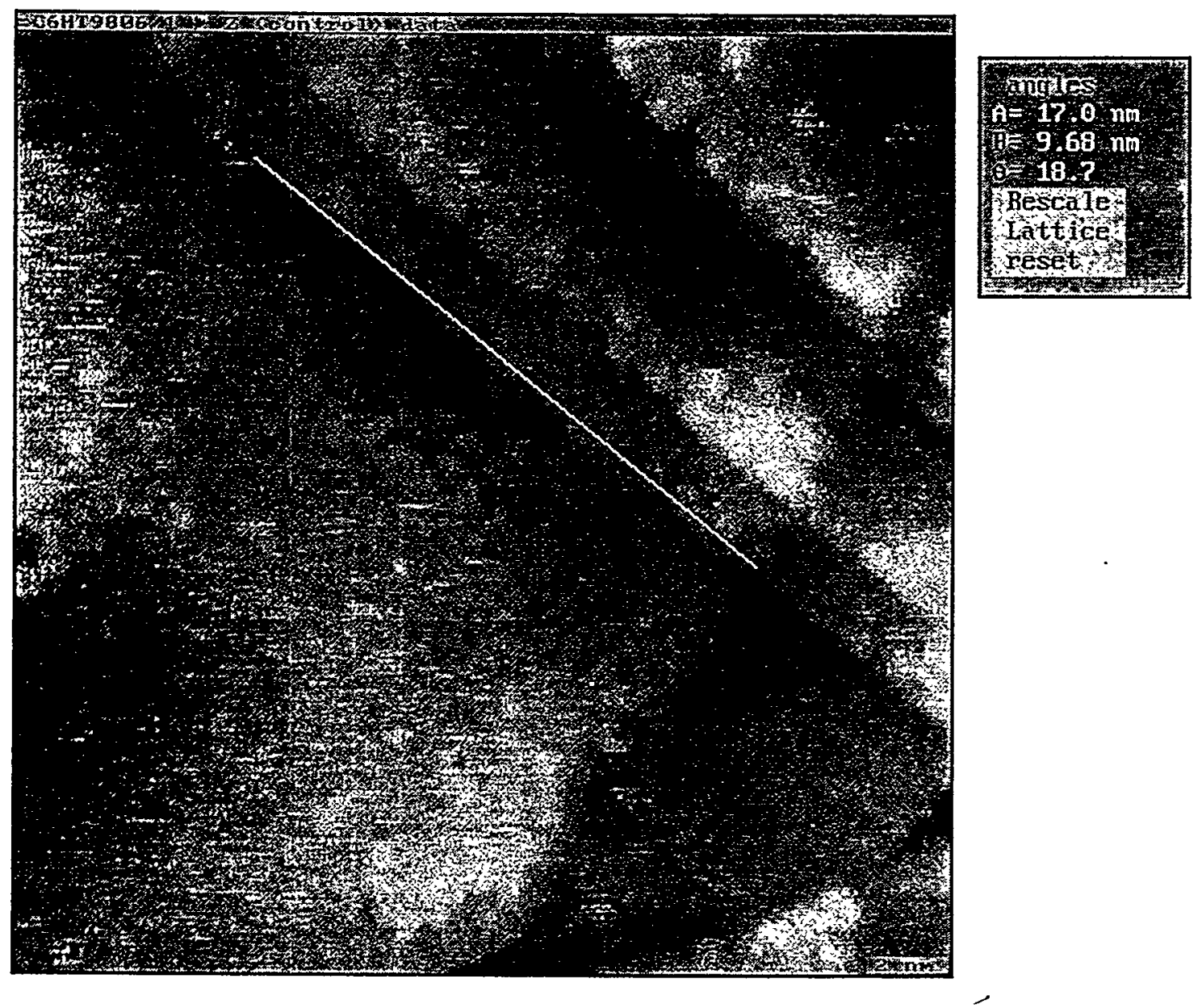

Figure 7-5: The second type of ordered domains formed by the adsorption of cyclohexene. The angle of rotation of the domain with respect to the step edges is approximately $19^{\circ}$. 


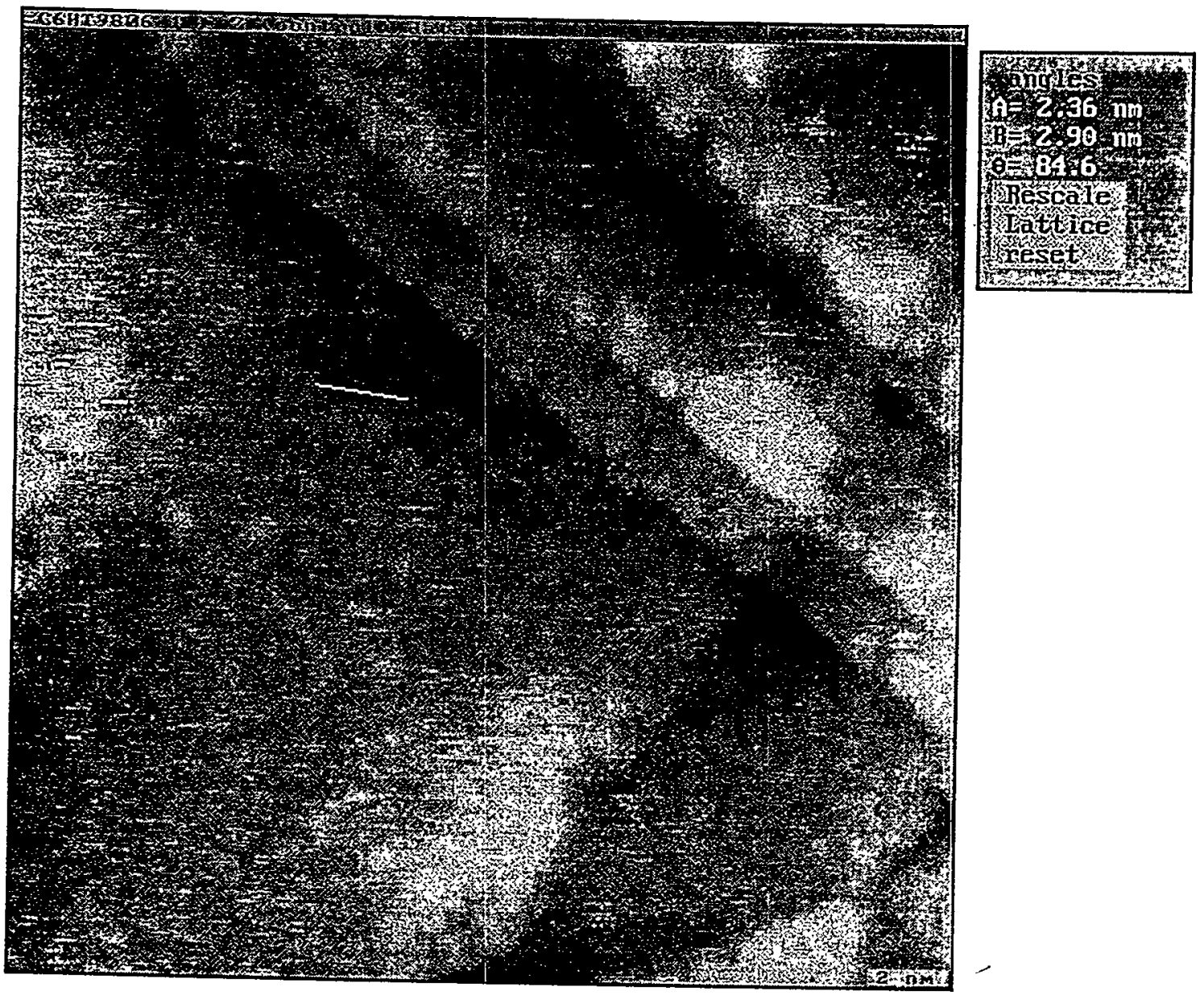

Figure 7-6: A topographic image of the overlayer showing the size of the unit cells.

Each line is two unit cells long. 


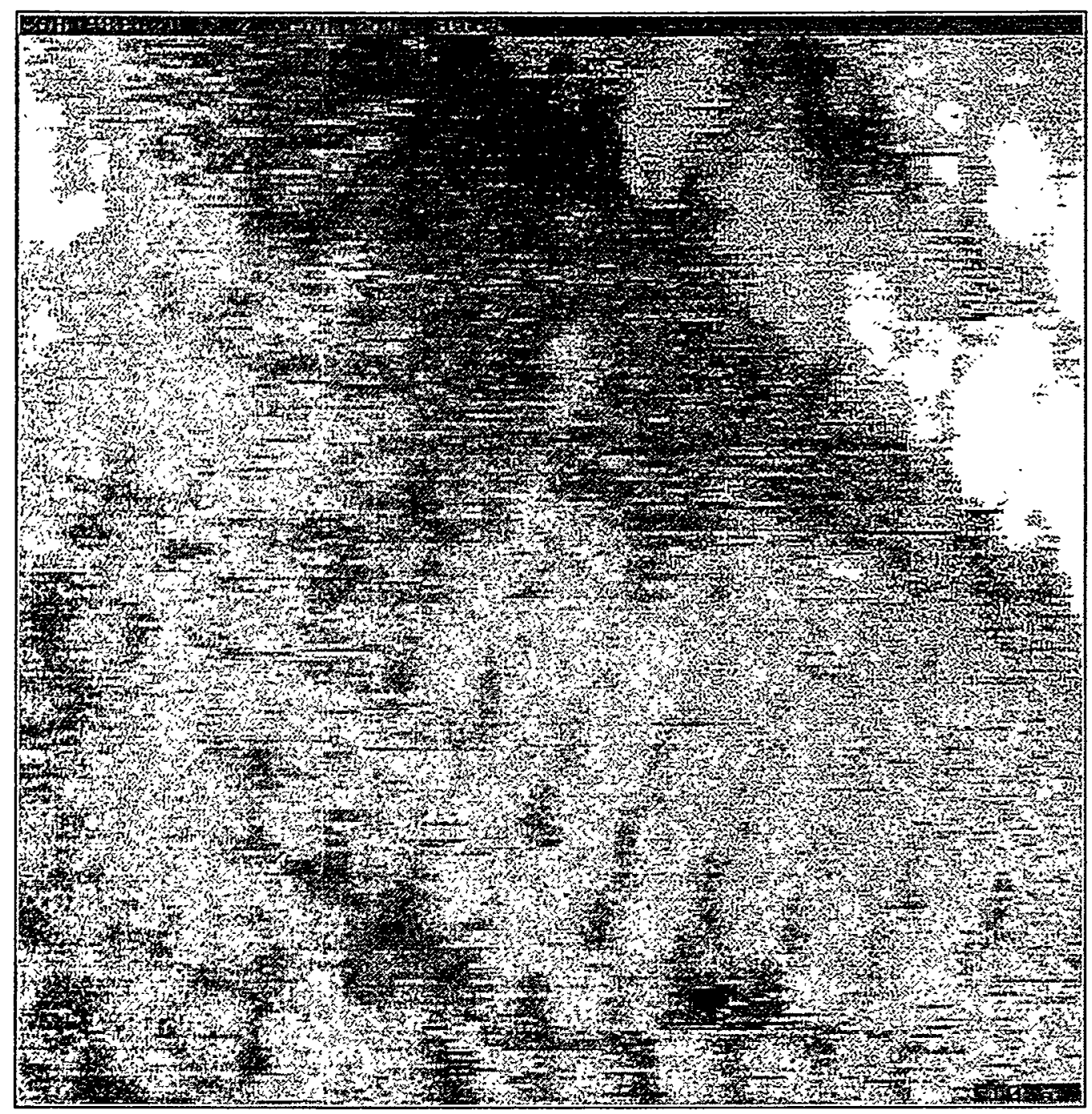

Figure 7-7: An image taken at low bias voltagee $(25 \mathrm{mV})$ which shows the ringed nature

of the adsorbed molecules. 
LEED, but no ordered LEED patterns were observed. STM images, however showed that there were ordered domains on the metal surface. Images of these ordered domains are shown in Figure 7-8. Again, we can see that these domains are rotated with respect to the close-packed directions of the rhodium substrate by approx. $19^{\circ}$.

There is some indication that the 1,4-cyclohexadiene may be spontaneously dehydrogenating to benzene when it adsorbes onto the surface. In order to block this reaction, experiments were done where the surface was exposed to a constant background pressure of $5 \times 10^{-8}$ torr of $\mathrm{H} 2$ before the 1,4 cyclohexadiene was adsorbed onto the surface. Ordered domains were also observed on the surface with the STM under these conditions. An example is shown in Figure 7-9. However, there were two distinct types of ordered domains on the surface. These two different domains differed both in the size of the unit cells (Figures 7-10 and 7-11) and in the corrugations of the molecules (Figure 7-12). One of the two domains had a unit cell of area $10.4 \AA \times 12.2 \AA$ with a corrugation of about $.6 \AA$. In contrast, the other type of domain has a unit cell with dimensions $13.1 \AA$ x 14.1

\section{Discussion}

There is evidence to suspect that cyclohexane and its derivatives will spontaneously dehydrogenate to benzene upon adsorption onto the rhodium surface. The studies of cyclohexane and cyclohexene on $\operatorname{Pt}(111)[3,4]$ shows that as temperature is increased the amount of cyclohexane or cyclohexene on the surface has increasingly been converted to benzene. At room temperature there is only benzene on the surface. 


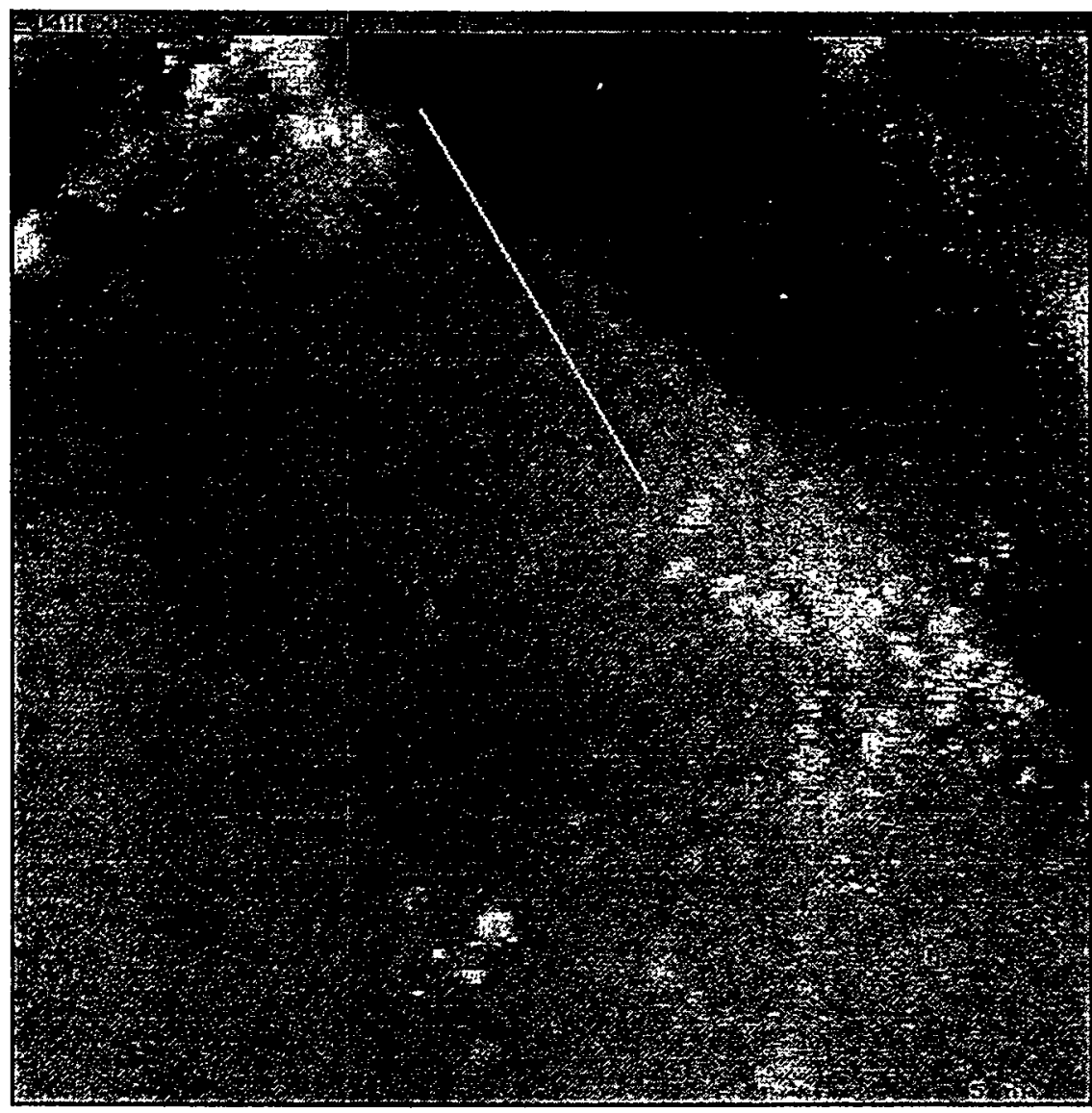

argas

$\mathrm{A}=14.6 \mathrm{~nm}$

$29.1 \mathrm{~nm}$

0.17 .0

Resciar

Lat $+2<\mathrm{c}$

coset

Figure 7-8: A topographic image of the surface after the adsorption of

1,4-cyclohexadiene. The domain is rotated by approximately $19^{\circ}$ 


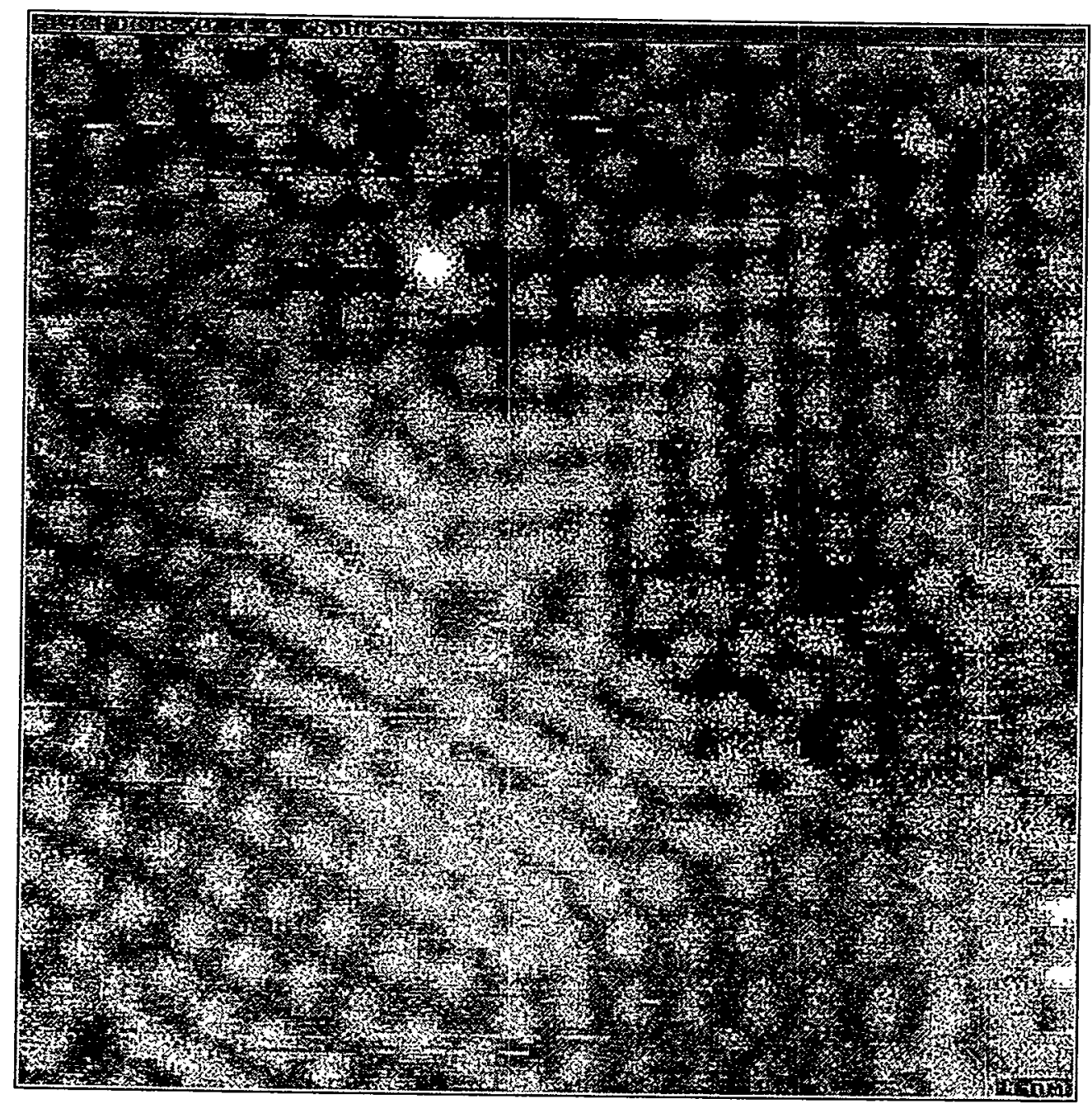

Figure 7-9: A topographic image of the surface after pre-adsoption of $\mathrm{H}$ atoms followed

by exposure to 1,4-cyclohexadiene. Two different unit cells are visible on the surface. 


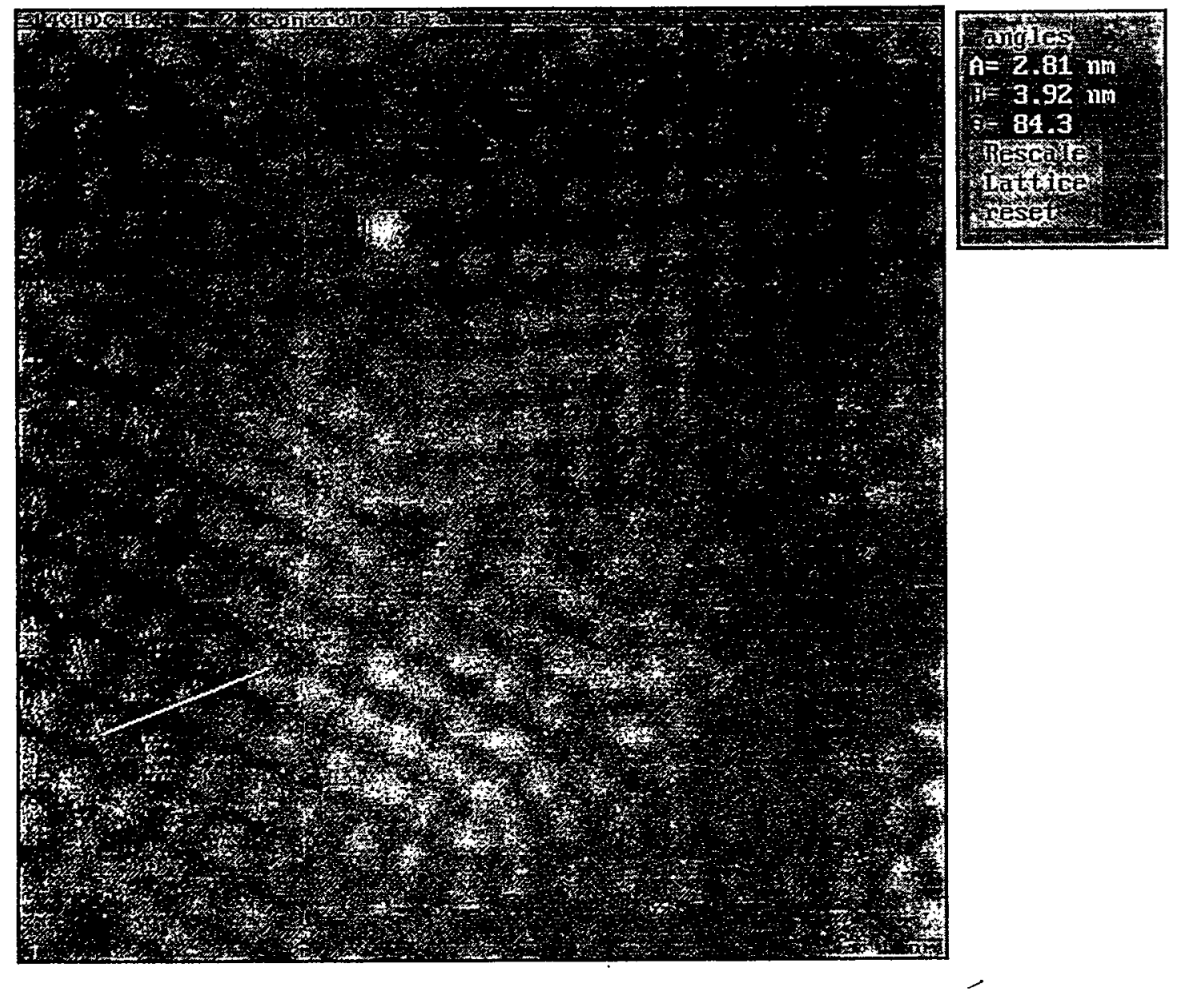

Figure 7-10: An image showing the sice of the larger unit cell. The white line is two unit cells long, while the dark line is three unit cells long. 


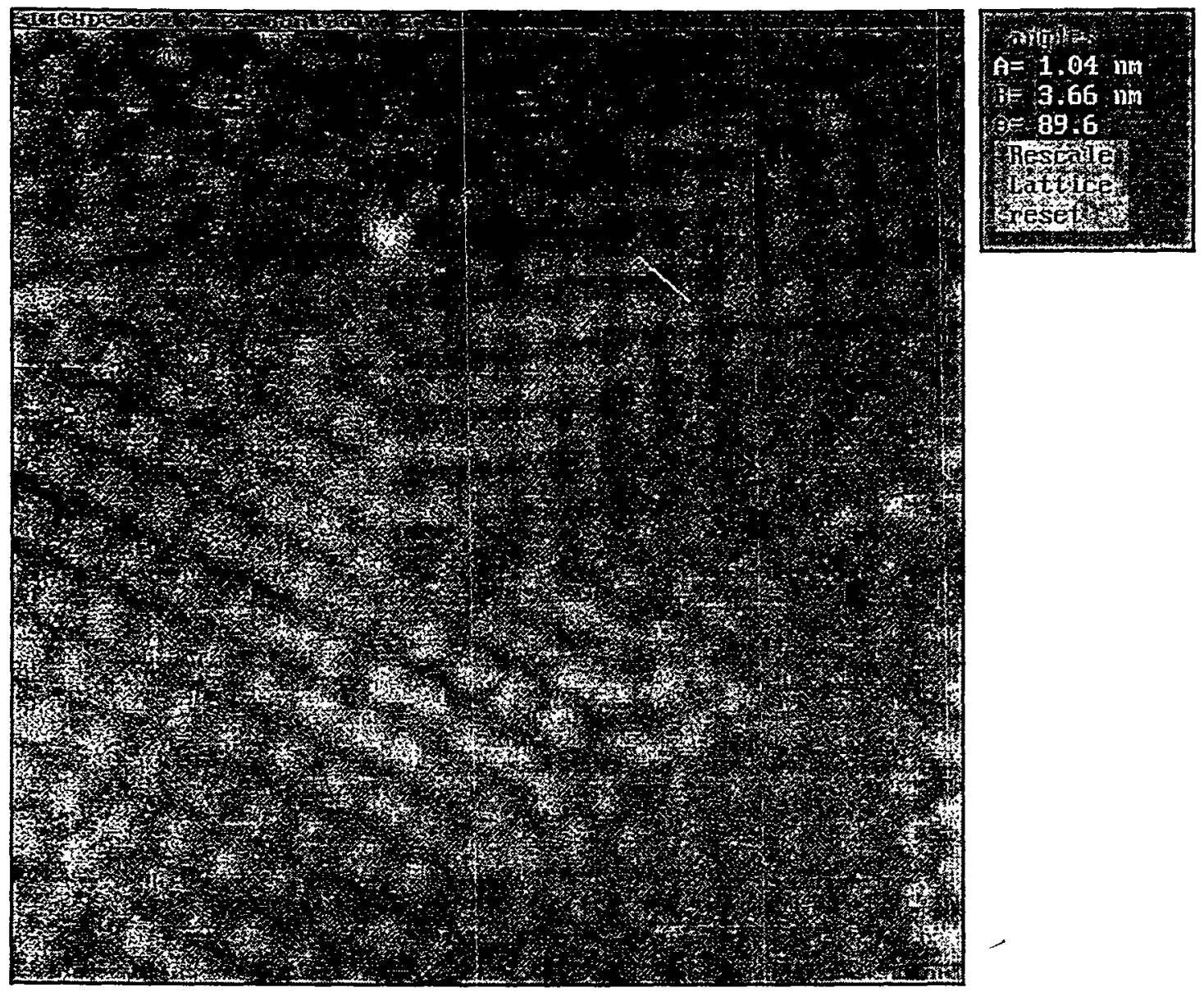

Figure 7-11: An image showing the size of the smaller unit cell. The white line is one unit cell long, and the dark line is three unit cells long. 

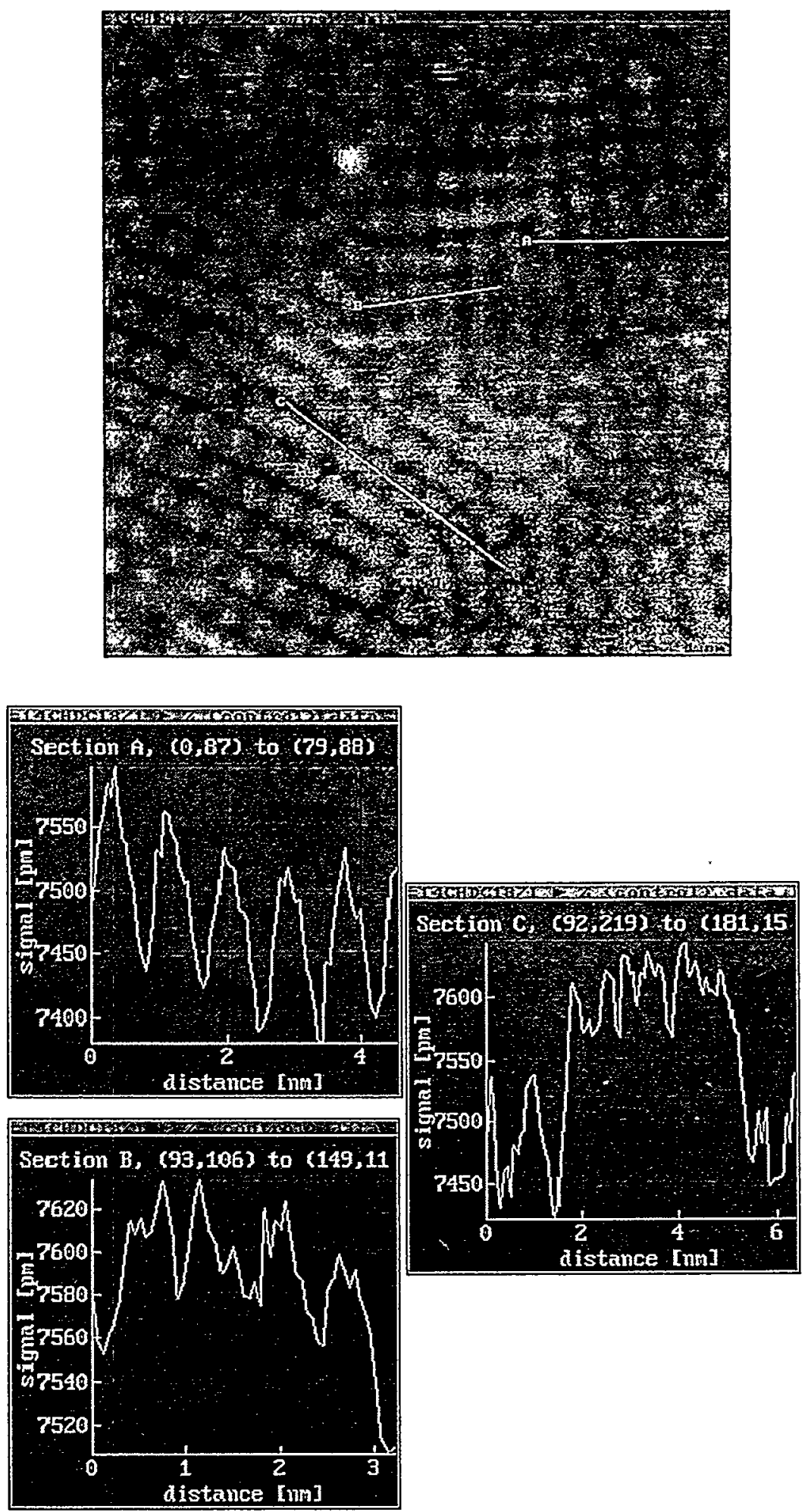

Figure 7-12: Three cuts of the surface show the corrugation of the molecules. The molecules in the larger unit cells have higher corrugation. 
Also, benzene is more strongly bound to rhodium than to platinum at room temperature, which can be seen in the difference in the diffusion rates of benzene on the two surfaces[7]. This stronger bond to the surface on Rhodium would mean that the conversion to benzene on rhodium is even more favorable thermodynamically than the conversion on the platinum surface. Given that the transition state for the conversion is likely to be similar, the kinetic favorability is also most likely not to have a significant effect on the conversion. With both kinetic and thermodynamic considerations for the conversion to benzene at least as favorable on rhodium as on platinum, it stands to reason that the conversion to benzene at room temperature which occurs on platinum will also occur on rhodium.

The images of cyclohexene adsorbed on the $\mathrm{Rh}(111)$ surface form two different structures. The $(3 \times 3)$ overlayer structure is evident in both LEED experiments as well as in the STM images. The second structure observed in the cyclohexene experiments is rotated with respect to the substrate. This is true of the $c(2 \sqrt{3} \times 4)$ overlayer structure. It is interesting to note that both the $(3 \times 3)$ and $c(2 \sqrt{ } 3 \times 4)$ structures are structures formed by benzene when adsorbed on the rhodium (111) surface[7]. Also, the molecules observed in the STM images seem to have a high degree of symmetry. We would expect this of benzene, since it has six-fold symmetry in the gas phase, and has been observed to have three-fold symmetry on the Rh (111) surface [7]. Cyclohexene, however, should have very low symmetry, since there is only one double bond in the ring. This lone double bond should exhibit a different tunneling probablilty than the rest of the molecule and should therefore show up as a bump or a depression in the molecule (this depends on whether the state which is accessible to the tunneling electrons is the pi bonding orbital or 
the pi anti-bonding orbital). Since such a feature is not observed in the STM images, this is added evidence that that cyclohexene has dehydrogenated to form benzene on the rhodium surface.

The 1,4-cyclohexadiene adsorbed on the surface shows similar behavior to the cyclohexene. The ordered domains are rotated with respect to the surface by $19^{\circ}$. This is indicative of the $c(2 \sqrt{3} \times 4)$ structure of benzene. The size of the unit cell is also similar to that of the $c(2 \sqrt{3} \times 4)$ unit cell exhibited by the adsorption of cyclohexene on the metal surface.

In the case of the pre-adsorption of $\mathrm{H}_{2}$, the $\mathrm{H}_{2}$ molecule dissociatively adsorbs on the rhodium surface [8,9]. If, upon adsorption, 1,4-cyclohexadiene spontaneously dehydrogenates to form Benzene plus two $\mathrm{H}$ atoms on the surface, then the preadsorption of $\mathrm{H}_{2}$ to form $\mathrm{H}$ atoms on the surface should inhibit the reaction. The $\mathrm{H}$ atom adsorption sites on the rhodium surface will fill with the dissociative adsorption of the $\mathrm{H}_{2}$. When the 1,4-cyclohexadiene adsorbs, the $\mathrm{H}$ atoms formed by the conversion to benzene will not be able to sit in their preferred sites and will have to fill higher energy sites. This will disadvantage the dehydrogenation thermodynamically and may prevent the reaction from occurring.

The STM images of 1,4-cyclohexadiene show an ordered overlayer with two distinct types of domains on the surface, with two different unit cell sizes. Unlike the case of cyclohexene where both the $(3 \times 3)$ and $c(2 \sqrt{3} \times 4)$ were evident (during different experiments) it is clear that neither of these domains correspond to the ( $3 \times 3)$. If the one of these domains had the $(3 \times 3)$ structure then each molecule in the domain would be surrounded by six other molecules at the same distance while these structures have four 
nearest neighbors. This is due to the size of the included angle in the $c(2 \sqrt{3} \times 4)$ cell being larger than $60^{\circ}$, meaning that fewer than six molecules can fit into the $360^{\circ}$ around the central molecule. The included angle of the (3x3) unit cell is exactly $60^{\circ}$, so that there are six nearest neighbors to the central molecule in the unit cell. Simple inspection of the images shows that there are fewer than six nearest neighbor molecules in the ordered arrangement. Therefore, this structure be the $(3 \times 3)$ structure.

While the $(3 \times 3)$ domain can be removed as a possible surface structure, the larger of the two unit cells corresponds well with the $c(2 \sqrt{3} \times 4)$ structure. The symmetry of the unit cell as well as the size is similar to the $c(2 \sqrt{3} \times 4)$ structures seen in the adsorption of 1,4-cyclohexadiene without $\mathrm{H}_{2}$ pre-treatment, as well as the $\mathrm{c}(2 \sqrt{3} \times 4)$ structure seen in the STM images of the adsorbed cyclohexene. The smaller of the two unit cells is not readily classifiable. It may be a previously unobserved structure for benzene which only occurs because of the adsorption of hydrogen on the surface. Another possibility is that this structure consists of 1,4-cyclohexadiene adsorbed on the surface. Thé STM images in this study show no conclusive evidence of what molecular species is in this smaller domain. Spectroscopic studies would likely be able to help with this identification. 


\section{References}

[1] G. D. Waddill and L.L. Kesmodel, Chem. Phys. Lett., 128, 1986, p208.

[2] J. L. Gland, K. Baron, and G.A. Somorjai, J.of Catalysis, 36, 1975, p305.

[3] D. Land, W. Erley. and H. Ibach, Surf. Sci., 289, 1993, p237.

[4] M.A. Chesters and S.F. Parker, and R. Rival, J. Electron Spectrosc.

Relat. Phenom., 39, 1986, p155.

[5] F.M. Hoffman, T.E. Felter, P.A. Thiel and W.H. Weinburg, Surf. Sci., 130, 1983, pl73.

[6] H.A. Yoon, personal communication.

[7] H. A. Yoon, M. Salmeron, and G. A. Somorjai, Surf. Sci. 376, 1997, p.254.

[8] J. T. Yates, P. A. Thiel, and W. H. Weinberg, Surf. Sci. 84, 1979, p.427.

[9] D. F. Padowitz and S. J. Sibener, J. Vac. Sci. Technol. A 9 (4), 1991, p.2289. 


\section{Chapter 8: Para- and Meta- Xylene}

\section{Introduction}

The structure of adsorbed organic molecules is an important topic in surface chemistry [1-9]. The scanning tunneling microscope (STM) can provide information about the structure and adsorption site of molecules in both ordered and disordered layers. For poly-atomic molecules, an additional question that involves the spatial resolution limit of the STM is whether the internal shape of such molecules can be resolved in order to distinguish between different molecules and to determine if changes in the structure can be detected that might reflect changes in binding properties [10-12]. For example, in the case of benzene, the molecule has been imaged under appropriate conditions as a toroidal object, reminiscent of the ring structure associated with the $\pi$ orbitals that one might expect from the aromatic molecule. We thus explored whether similar internal structure can be resolved for other molecules, such as xylenes, which are substituted benzenes with 2 methyl groups substituting 2 of the hydrogen atoms.

In this paper, we explore the surface structures formed by para- and meta-xylene adsorbed on $\mathrm{Rh}(111)$ at room temperature by STM. Several systems have been studied on 
this crystal face by STM, including CO [13], sulfur [14] and benzene [15]. As we shall see, long-range order is difficult to achieve and only relatively small domains of ordered molecules form in the case of para-xylene, while for meta-xylene no ordered domains could ever be observed. Despite this, and due to the high spatial resolution of STM, structural information can be obtained. In particular, the molecular images have distinct shapes that permit easy identification and the determination of their orientation relative to the substrate. In order to facilitate understanding of the results, it is helpful to consider the van der Waals shapes and dimensions of these two molecules, which we present schematically in Figure 8-1.

\section{Experimental}

The experiments were carried out in a stainless steel ultra-high vacuum (UHV) chamber with a base pressure of $6 \times 10^{-10}$ torr. The chamber is equipped with low energy electron diffraction (LEED), Auger electron spectroscopy (AES), ion sputtering, mass spectrometer for residual gas analysis and STM.

In all of our experiments, $90 \%$ platinum-10\% rhodium tips were used. No significant changes of the molecular shapes were observed as a function of tunneling conditions, except at very small gap resistance where the tip-induced molecular displacements and overall image degradation occurred. For this reason, and unless otherwise noted, the images were taken with a gap resistance of $100 \mathrm{M} \Omega$ or higher. 

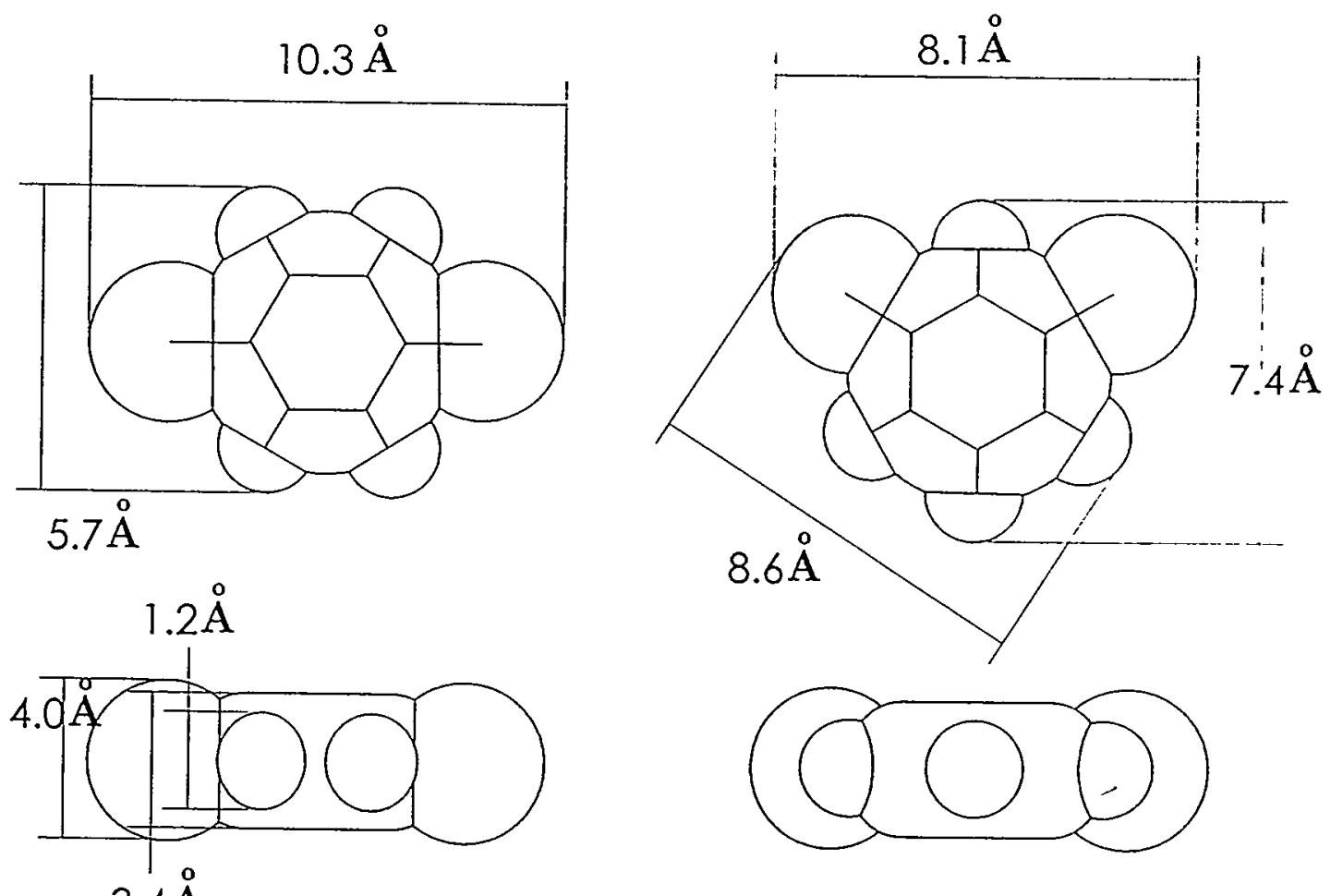

Figure 8-1: A schematic drawing of para- and meta-xylene based on the van der Waals radii of the constituent atoms. 
The rhodium crystal was oriented parallel to the (111) direction within 1-2 degrees. The misalignment produced terraces of an average width of $150 \AA$. It was cleaned by repeated cycles of argon ion sputtering $\left(5 \times 10^{-5}\right.$ torr of $\left.\mathrm{Ar}\right)$, followed by annealing in oxygen $\left(5 \times 10^{-8}\right.$ torr of $\left.\mathrm{O}\right)$ and vacuum. Cleanliness of the sample was determined by AES. Para-xylene (99\% pure) was obtained from Acros Chemical, and meta-xylene from Fisher Scientific. Prior to introduction in the vacuum chamber, they were subjected to several freeze-pump-thaw cycles to remove water and other contaminants. When the xylenes were analyzed with the mass spectrometer, the observed fragment ion distribution showed no major contaminants. Each xylene isomer was introduced into the UHV chamber using a separate variable leak valve. The valves were connected to a tube doser to allow the organic vapor to directly impinge on the sample. Before exposure, the sample was flashed to $700 \mathrm{~K}$ and allowed to cool to $300 \mathrm{~K}$. The sample was then positioned within $1 \mathrm{~cm}$ of the tube doser for exposure to the xylene. Exposures varied from $.05 \mathrm{~L}$ to $5 \mathrm{~L}$, depending on the nature of the experiment. After exposure, the base pressure returned to $6 \times 10^{-10}$ torr after a few minutes. No annealing was carried out after exposure to xylene.

In coadsorption experiments, exposures were sequential, not concurrent. Paraxylene was first adsorbed on the surface at an exposure of $.02 \mathrm{~L}$ and then the sample was exposed to $.05 \mathrm{~L}$ of meta-xylene. 


\section{Para-xylene}

Typical STM images of the para-xylene covered surface are shown in Figures 8-2 and 8-3. They were observed for a variety of exposures from $.05 \mathrm{~L}$ to $5 \mathrm{~L}$. Individual molecules appear as diamond-like shapes in the images. They form ordered domains with three different orientations $120^{\circ}$ apart. The domain size is variable with an average diameter of $70 \AA$, usually containing about 50 molecules. The molecules are oriented with their long axis along the [112] and similar directions, i.e., forming a $30^{\circ}$ angle to the close-packed directions of the surface. This is visible in Figure 8-3, with the help of the straight [110]-type steps, which are the result of emerging (111)-type slippage planes, seen in larger images. The steps cross each other at the expected angles of $60^{\circ}$ or $120^{\circ}$. The molecules are packed such that they line up vertex-to-vertex, parallel to the long diagonal of the diamond shape. The unit cell is a rhomboid with an angle of $80^{\circ}$ and sides of 8.4 and $6.9 \pm 0.5 \AA$. The distance between the molecules along the diagonal is $10.0 \pm$ $0.5 \AA$. These distances are in agreement with a dense packing of molecules with van der Waals dimensions such as those in Figure 8-1. Unfortunately we were not able to image simultaneously both the molecules and substrate rhodium atoms, so the registry with the substrate could not be determined.

There are also many areas of the monolayer where the xylene molecules are disordered. These regions account for $30-50 \%$ of the imaged area.

While the xylene molecules are not diffusing on the surface on these dense layers, some molecular motion is observed for the molecules near the domain edges or in the 

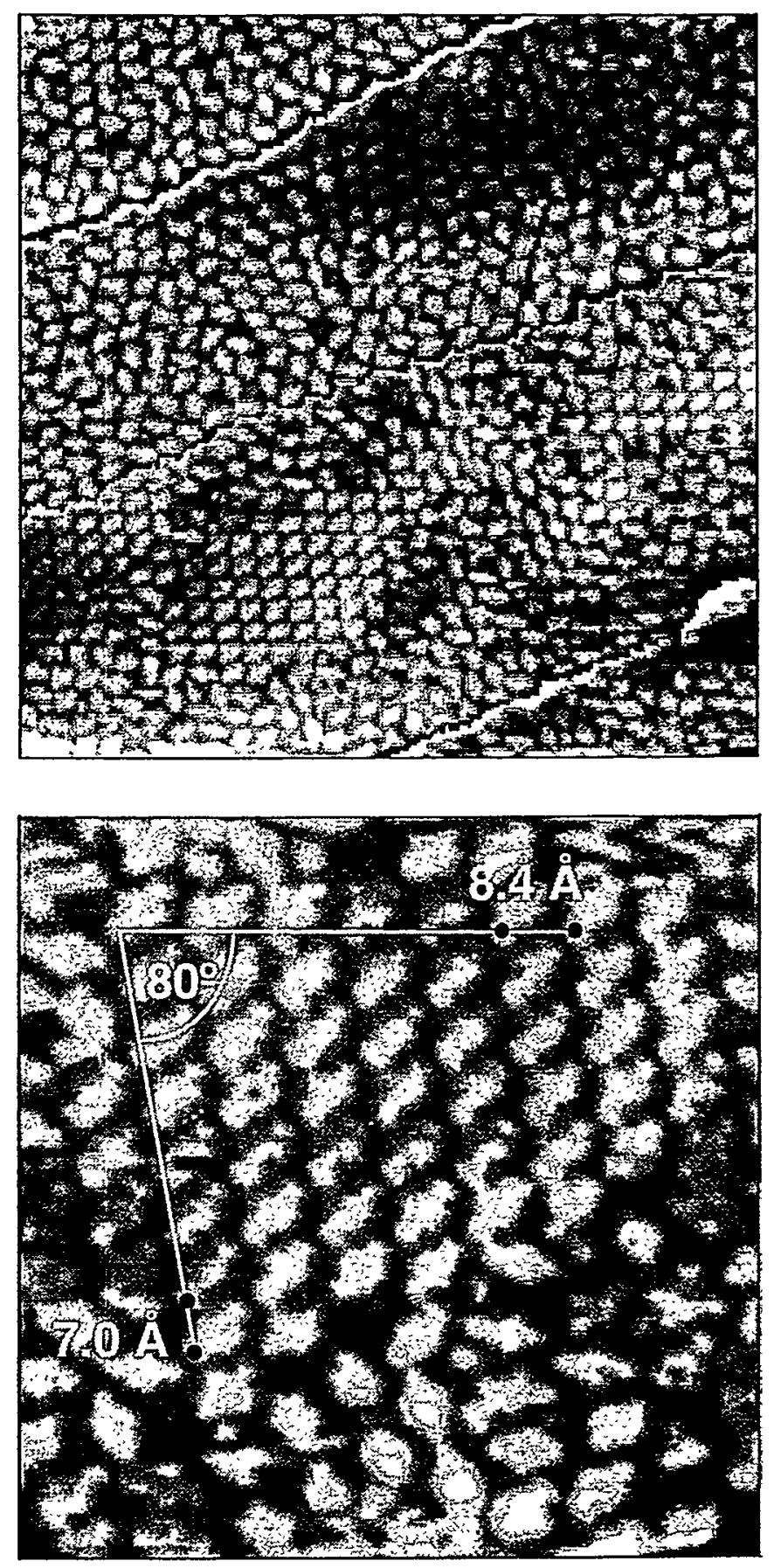

Figure 8-2: Top: A topographic image of an adsorbed layer of para-xylene on $\mathrm{rh}(111)$.

Bottom: A detailed view of an ordered domain. 

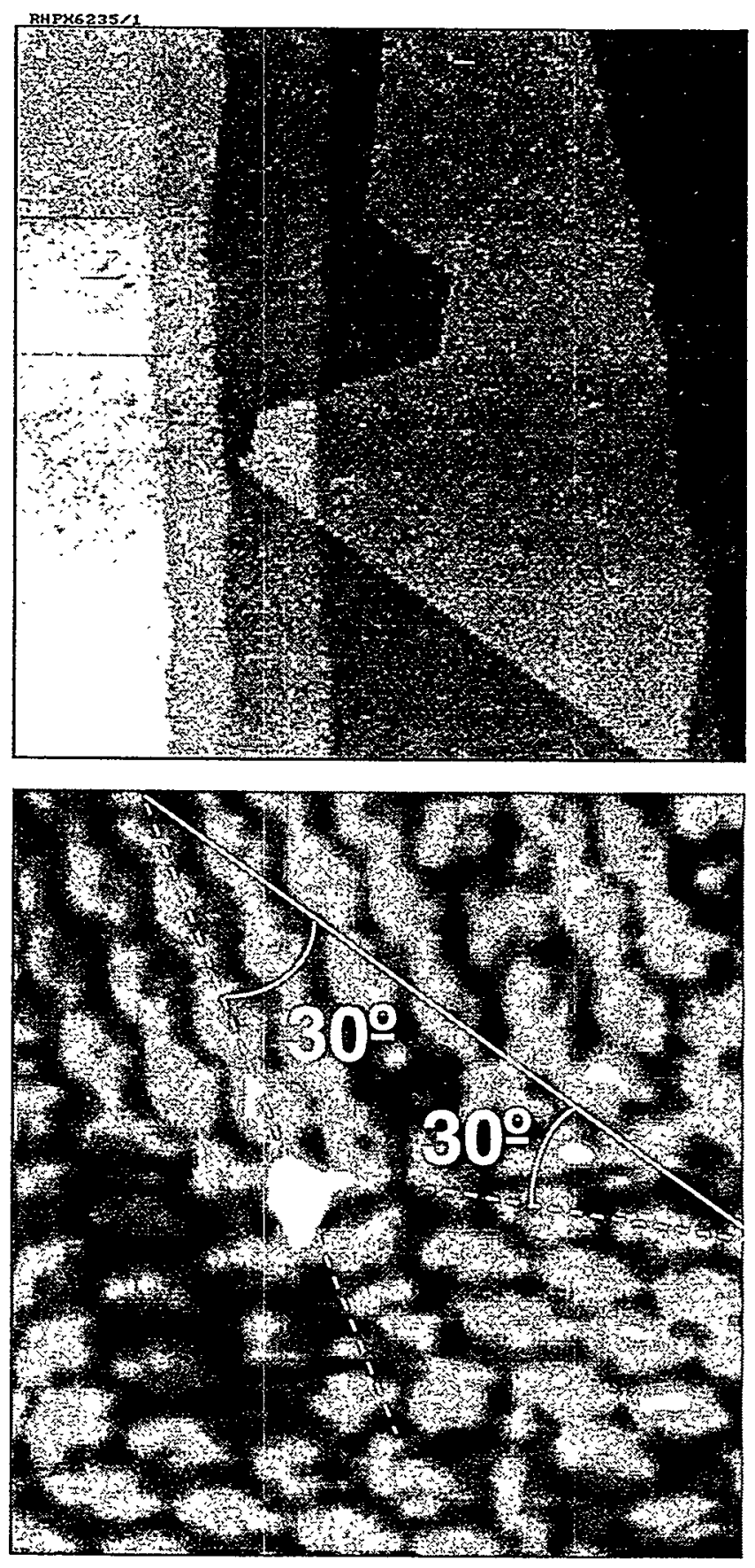

Figure 8-3: Top: A topographic image of the surface saturated with para-xylene. Bottom: A detailed view which show the angle of the molecules with respect to the straight step edges in the larger image above. 
disordered areas. The motion of these molecules is mostly that of flipping into one of the other two preferred orientations with possibly some small displacements.

At low coverage, obtained with less than $.05 \mathrm{~L}$ exposure, para-xylene adsorbs preferentially on the top of the step edges, as shown in Figure 8-4. No stationary paraxylene molecules are observed on the terraces under these conditions. However, there are features on the terrace in the form of streaks, with a similar apparent height as that of para-xylene molecules. These streaks are due to the diffusion of molecules on the surface at a rate faster than can be resolved at our scanning speed of $50 \AA / \mathrm{sec}$. This behavior has been observed in other systems of aromatic molecules adsorbed on noble metals [17].

In the better-resolved images, internal structure can be discerned inside the diamond-shaped features, as shown in Figure 8-5. The corners opposite the long diagonal are brighter, i.e., the tunneling probability is higher there than in the rest of the molecular image. The center part, where the ring should be located, appears dimmer. Typical profiles across the two diagonals are shown in the figure. Often the intensity distribution is not symmetric, an effect that we ascribe to tip asymmetry.

\section{Meta-xylene}

Meta-xylene yields STM images like those shown in Figure 8-6, which corresponds to a saturation coverage obtained after exposures above $0.1 \mathrm{~L}$. The molecule appears as a triangular feature in the STM images. In contrast to para-xylene, no ordered structure is ever observed. However, the molecular shapes are always oriented with one side parallel to the close-packed rhodium rows, as determined from the alignment of straight [1 10$]$ type steps from emerging bulk dislocations (Figure 8-6). 


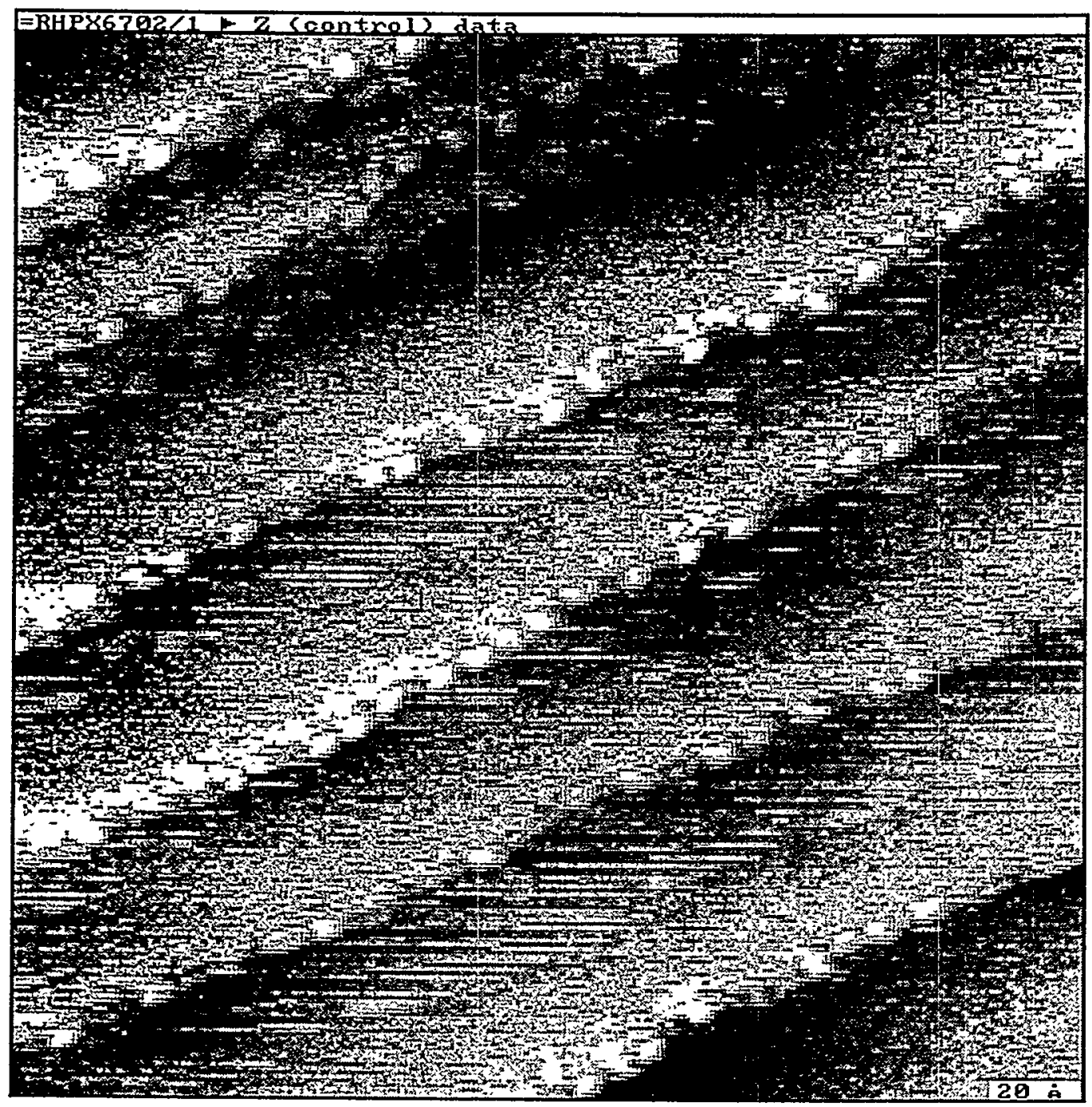

Figure 8-4: An image of the low-coverage para-xylene (0.02L). Immobile molecules of para-xylene are imaged at the step edges. 

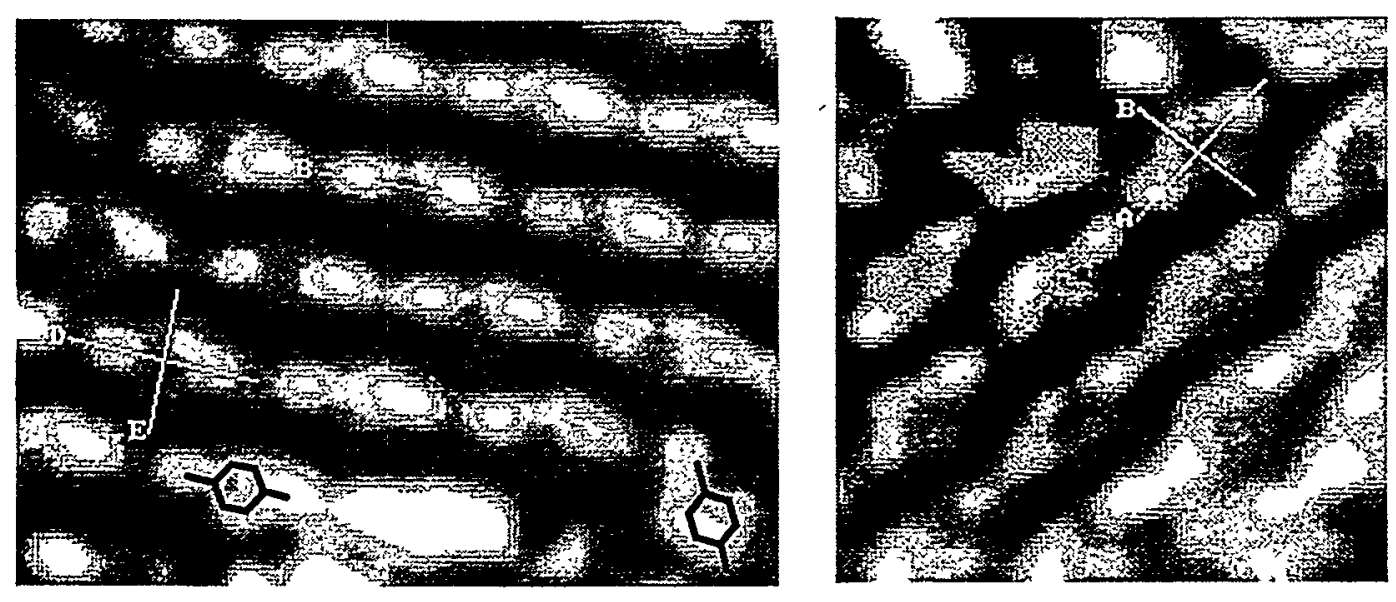

\section{Section A, D}

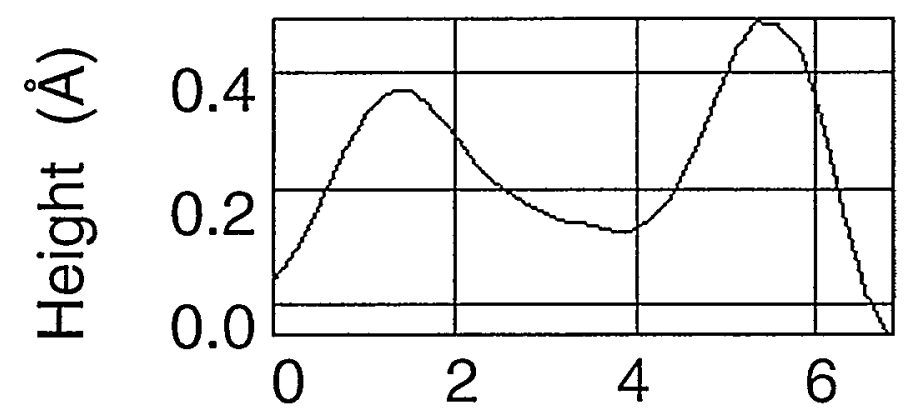

\section{Section $B, E$}

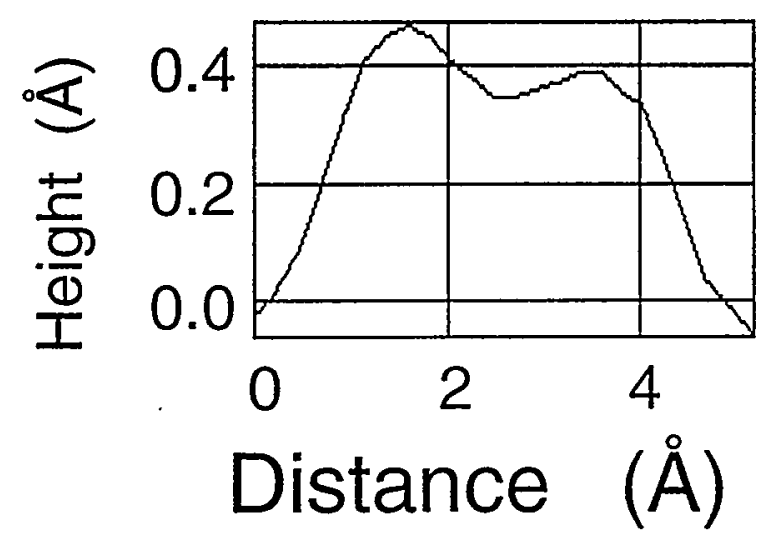

Figure 8-5: Topographic images of para-xylene on $\mathrm{Rh}(11)$. The aromatic ring gives rise to the central depression in the molecules. The cursor profiles are shown at the bottom of the figure. 


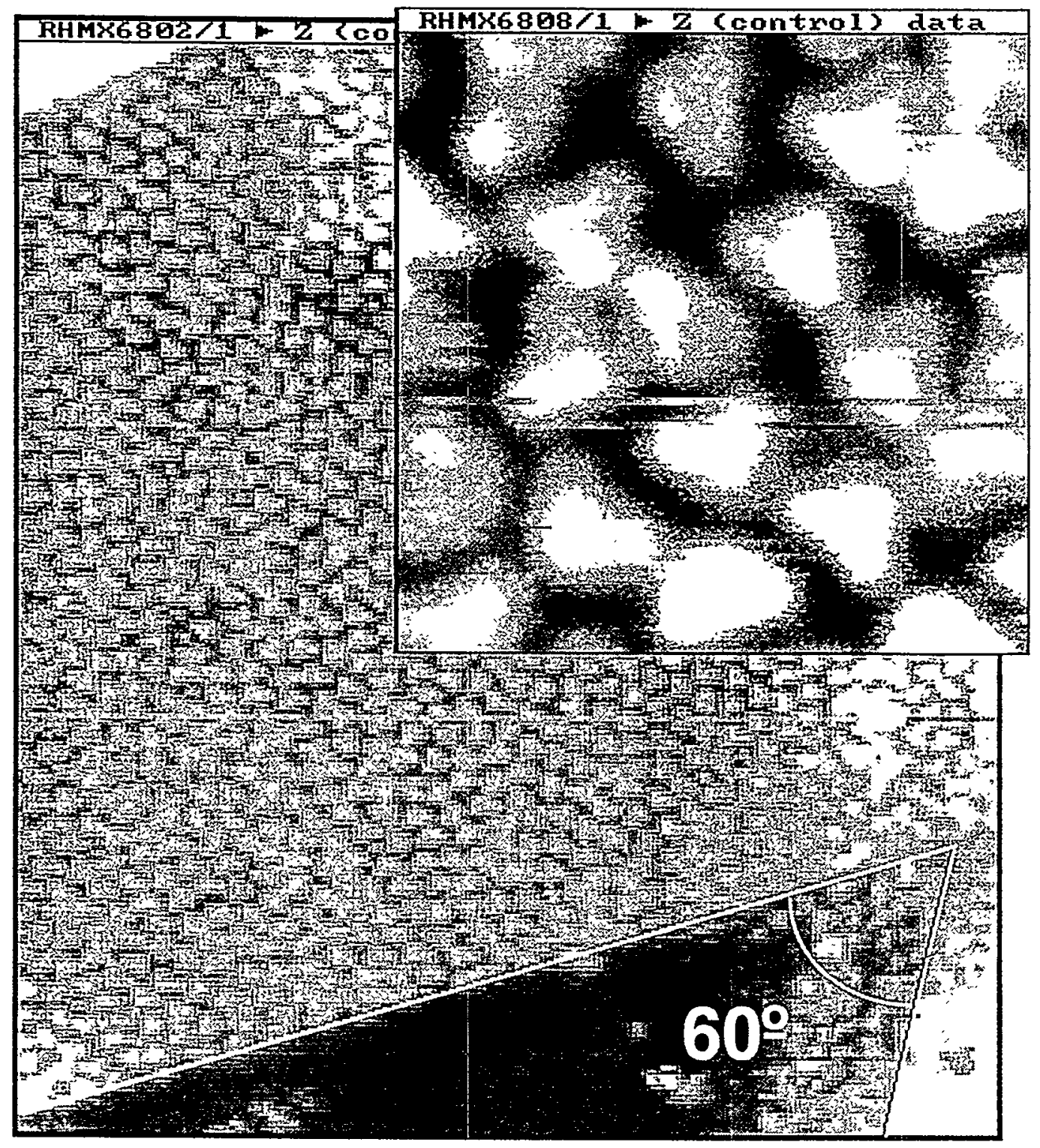

Figure 8-6: A topographic image of meta-xylene on the $\mathrm{Rh}(111)$ surface. The image shows the orientation of the molecules with respect to the step edges. 
As for para-xylene, the meta-xylene molecules are mostly immobile on the scanning time scale. However, some occasional motion of individual molecules can be seen in consecutive images (Figure 8-7). The images in this figure were taken one minute apart. For reference, a triangle was drawn with its vertices on three impurities. The arrows point to molecules that have changed their position or rotational orientation with respect to the stationary molecules on the surface. These moving molecules do not change their position by more than a few $\AA$ between images.

At low coverages (after exposures $<.1 \mathrm{~L}$ ), meta-xylene adsorbs preferentially at the top of the step edges, as shown in Figure 8-8. One side of the triangular images of the molecules is parallel to the steps. Similar to the case for para-xylene, no stable images of the molecules could be obtained on the terraces. Instead, there are features in the form of streaks along the scanning direction of similar height as the meta-xylene features. This is indicative of molecular diffusion during scanning.

No images of meta-xylene could be obtained that showed a central depression from the aromatic ring orbitals, as in the case of benzene and para-xylene. However, in the best resolved images, two corners of the triangle have higher corrugation than the third one, as shown in Figure 8-9. Symmetry arguments indicate that they correspond to either the location of the methyl groups or to the opposite corners of the benzene ring (see Figure 8-1). For reasons that will be discussed later, we have assigned them to the methyl groups in the sketch overlaid on the molecular images of the figure.

Interestingly, when meta-xylene is coadsorbed with $\mathrm{CO}$ following a low exposure of $.05 \mathrm{~L}$, diffusion of the meta-xylene molecules is inhibited, and they can be imaged on 


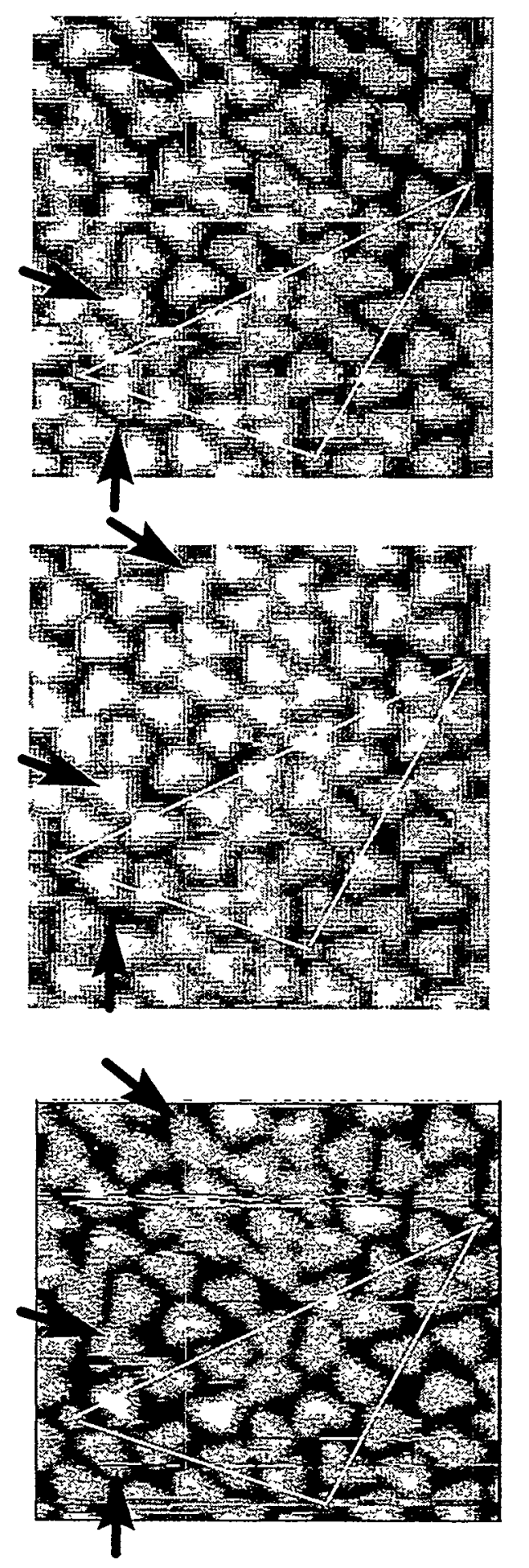

Figure 8-7: Three topographic images of meta-xylene showing the molecule that change position or orientations between scans. 

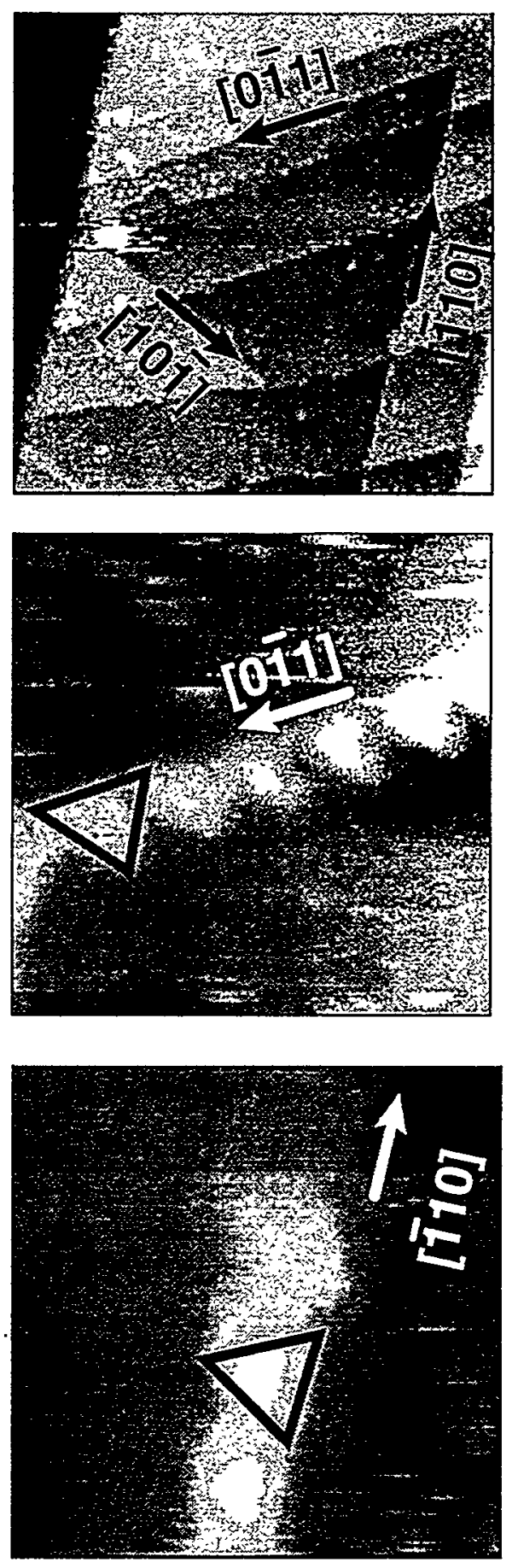

Figure 8-8: Topographic images of the surface after low exposure to meta-xylene. Top: A large scan image showing the orientations of the steps. Bottom: Details of how the individual molecules align with the steps. 


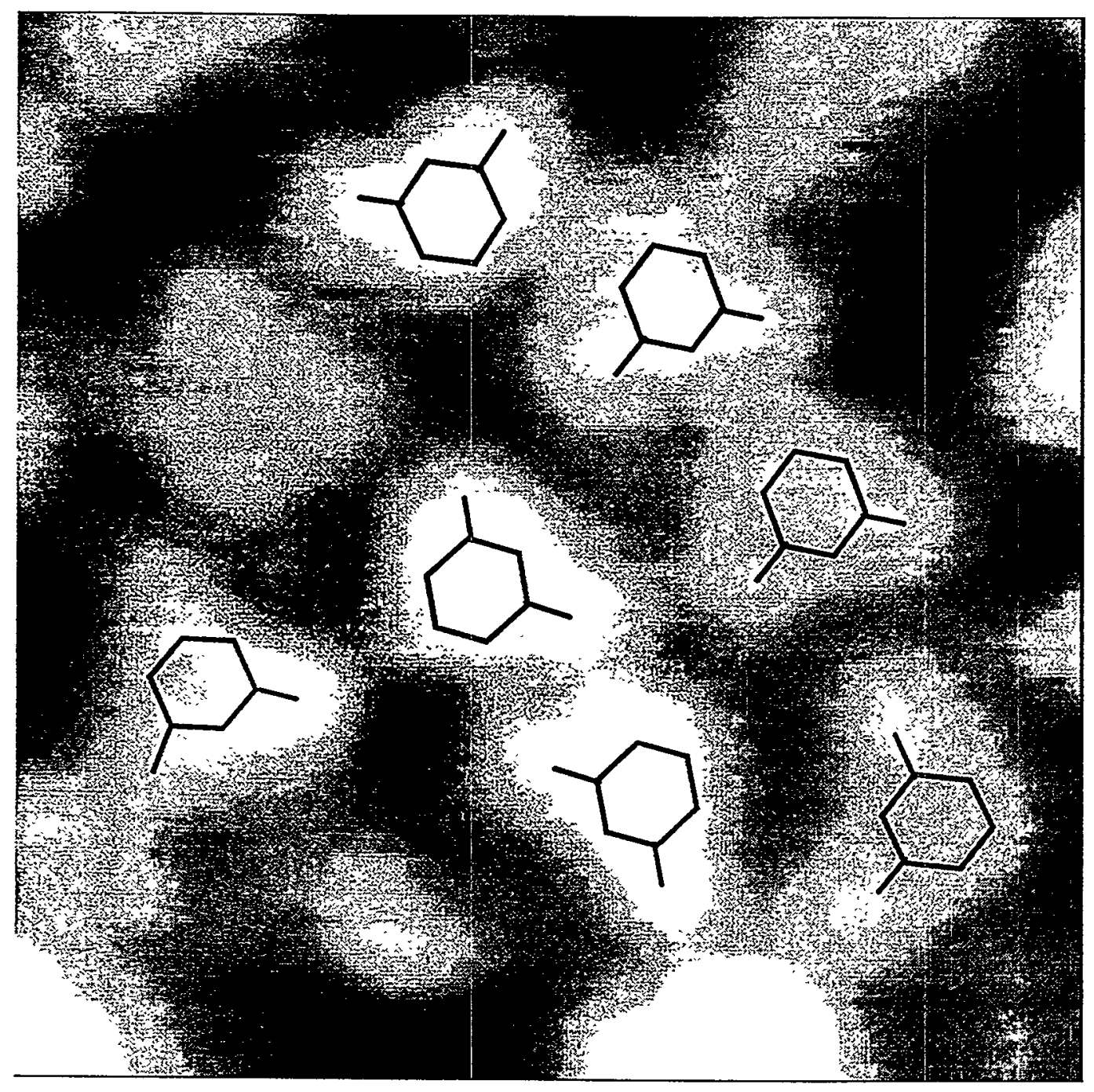

Figure 8-9: Close-up topographic image of meta-xylene. A model of the meta-xylene is superimposed on the molecules to show the orientation of the methyl groups. 

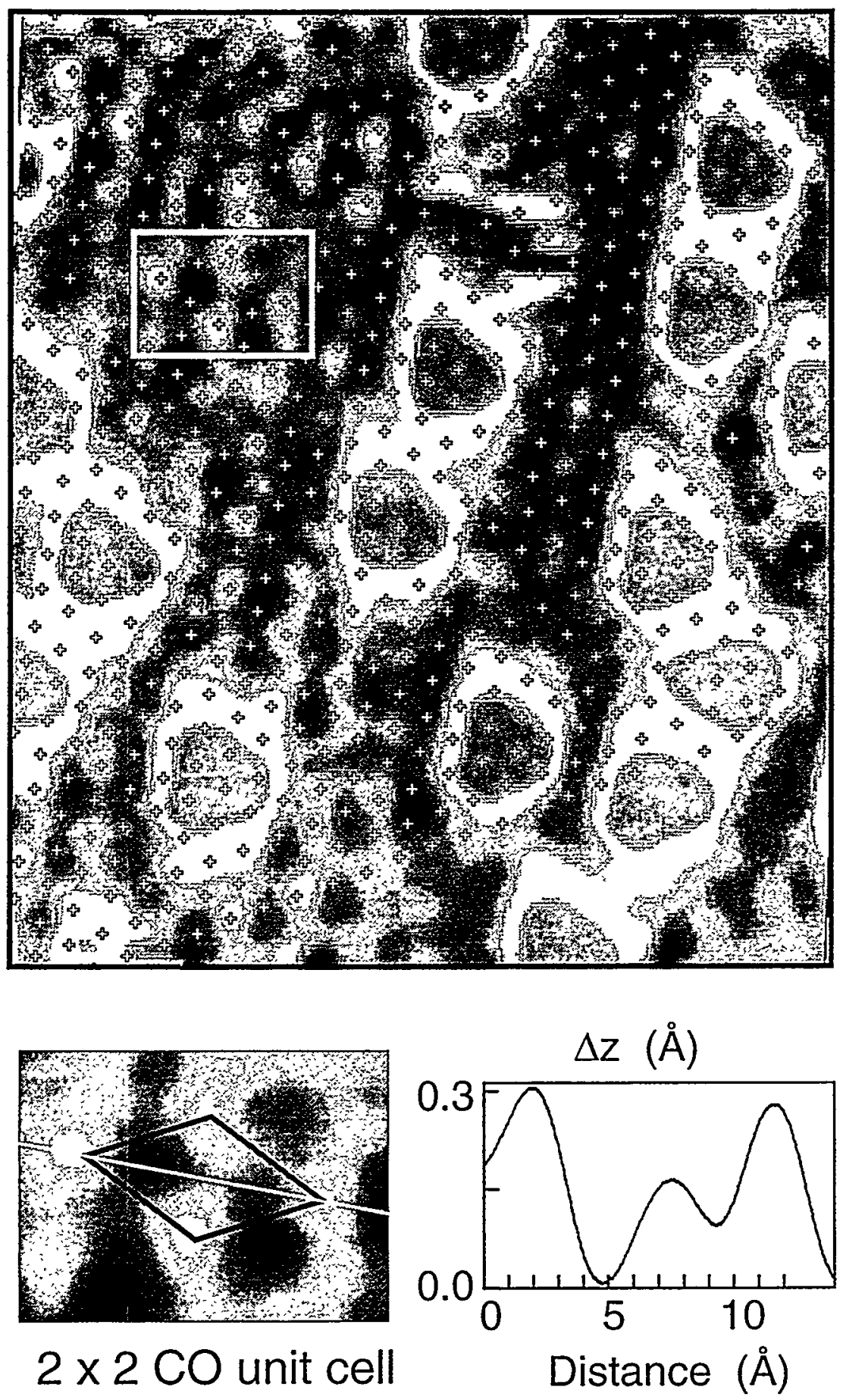

Figure 8-10: Topographic image of $\mathrm{CO}$ coadsorbed with meta-xylene at low coverage.

A lattice of crosses is superimposed to help determine the location of the xylene molecules. 
the terraces as well as at the step edges. Both the meta-xylene and the individual $\mathrm{CO}$ molecules are imaged at the same time, as shown in Figure 10. The $\mathrm{CO}$ molecules appear as a single bump with an apparent height of $0.25 \pm 0.1 \AA$, and the xylene molecules as a roughly triangular shape $0.6 \pm 0.1 \AA$ high. Since the $\mathrm{CO}$ forms an ordered structure, the positions of the meta-xylenes can be determined by their positions on the surface relative to the $\mathrm{CO}$.

\section{Meta- and para-xylene coadsorption}

When the sample is exposed to $0.02 \mathrm{~L}$ para-xylene, followed by an exposure to $0.05 \mathrm{~L}$ meta-xylene, a saturated layer of xylene molecules showing complete mixing is formed. No long-range order is observed. In Figure 11, the two isomers can be easily distinguished by their distinct shapes. Although para-xylene adsorbed before metaxylene, the step edges are not exclusively populated by the para-isomer. Some molecular motion is also taking place, visible by the presence of streaks due to molécules being scanned for only a few lines because of sudden rearrangements. Some of these effects might be induced by the tip, although we did not investigate this possibility in detail.

\section{Discussion}

We have seen that STM images produce para- and meta-xylene molecules with distinct shapes. Para-xylene is imaged as a diamond-shaped feature with a depression in the center, where the aromatic ring of the molecule should be located. Meta-xylene is imaged as a triangular shape, with no observable depression near its center. In both cases, 


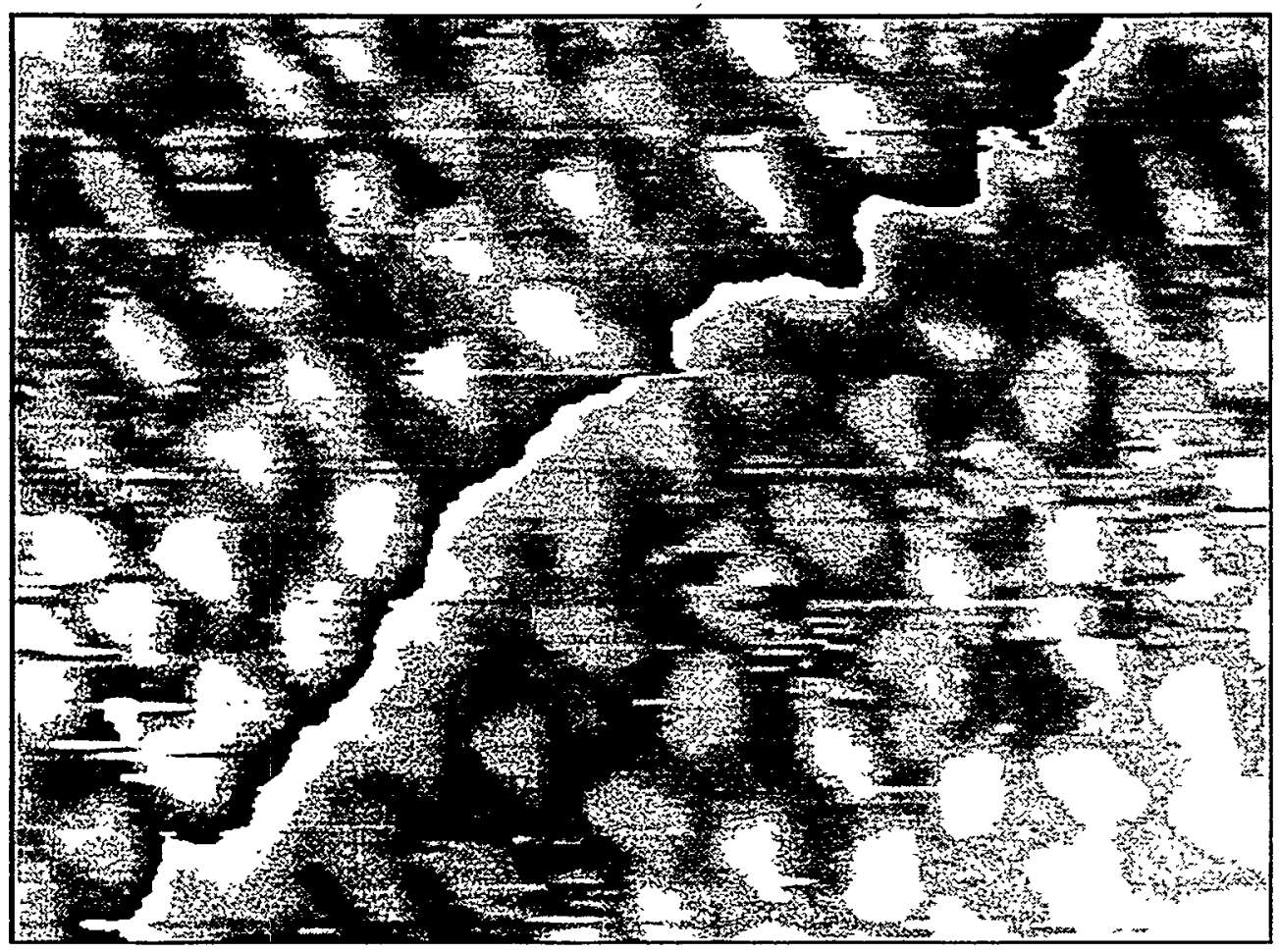

Figure 8-11: a topographic image of para- and meta-xylene coadsorbed on the rhodium surface. 
two bright spots are observed. For para-xylene, they are located at the opposite ends of the long diagonal of the diamond, while for meta-xylene they are on two triangular corners. Steric considerations indicate that the packing of para-xylene molecules in the ordered regions (see Figures 2 and 3) can only be achieved if the methyl groups are located at opposite ends of the long diagonal, which is parallel to the molecular rows (see Figure 1). The influence of the molecular electronic structure was taken into account in recent calculations of the STM images by Futaba et al. [18]. These authors found that the images of HOMO and LUMO orbitals indeed show a protrusion at the position of the $\mathrm{CH}_{3}$ groups, as we observed experimentally. At low coverage, para-xylene molecules are seen only at the top of the step edge. This is not unexpected, since many molecules have higher heats of adsorption at step edges on the Rh(111) surface [19].

Meta-xylene does not form an ordered domain structure on the Rh(111) surface under the present experimental conditions. However, the individual molecules appear to be oriented with one side of their triangular images parallel to the close-packed rhodium rows. The molecules do not seem to sit at any fixed distance relative to one another, but they maintain a center-to-center separation of 8-10 $\AA$, which is compatible with their van der Waals sizes. Like para-xylene, meta-xylene adsorbs preferentially at step edges at low coverage, most likely because of their higher binding energy at these sites.

While for exposures below $0.05 \mathrm{~L}$ both xylene isomers are stationary and readily imaged by STM at the step edges, there is evidence of diffusion on the atomic terraces. Small streak-like features with the same apparent height as the xylene molecules on the steps are observed in these images. These streaks are attributed to molecules that are imaged during a small fraction of the scan lines necessary to cover the whole molecule. 
The observation of stationary molecules only at step edges below saturation and covering the full surface at saturation for a wide range of exposures clearly points to diffusion on the terraces at low coverage. Also, at separate exposures of $0.02 \mathrm{~L}$ of para-xylene and of $0.05 \mathrm{~L}$ of meta-xylene, only molecules at the step edges are observed, which appear to cover less than $5 \%$ of the surface. However, when these exposures are made sequentially, saturation is observed. This could only happen if a significant number of molecules were already adsorbed on the terraces that were not imaged due to diffusion. Even in the case where molecular displacement is frustrated at high coverage due to steric hindrance, motions of para- and meta-xylene can be observed at the border of steps or in regions where packing is slightly smaller. This is visible by the simple inspection of successive images.

In the case of meta-xylene, it was possible to stop the diffusion of molecules at low coverage by co-adsorbing $\mathrm{CO}$. $\mathrm{CO}$ forms several ordered overlayers on the Rh(111) surface, including a (2x2) structure at 0.25 monolayers, when adsorbed by itself $[19,20]$. In our coadsorption experiments with meta-xylene, we also observed a (2x2) structure. Based on LEED and XPS data, Beutler et al. [19] assigned the CO adsorption site in this structure to the top site. Although the existence of the low-coverage (2x2) structure has been recently questioned based on a study of the isoteric desorption of $\mathrm{CO}$ [21], our present results confirm its existence. A more detailed study of the adsorption of $\mathrm{CO}$ on $\mathrm{Rh}(111)$ is given in chapter 5 . If we assume top site adsorption together with the STM images (see Figure 10), we can conclude that meta-xylene sits with its center near either bridge or three-fold hollow sites, assuming that the center of the triangular image corresponds to the center of the molecule. A 3-fold hollow site geometry would be 
similar to that found for benzene coadsorbed with $\mathrm{CO}$, according to a LEED dynamical study by Barbieri et al. [22]. However, in that study, $\mathrm{CO}$ was found to sit on hcp 3-fold hollow sites, which is not the position assumed for our $(2 \times 2)-\mathrm{CO}$ structure. This is interesting, since it indicates that the $\mathrm{CO}$ molecules not affected by xylene adsorption, i.e., adsorbed far away, stabilize the top site for $\mathrm{CO}$ even at the sites nearest to the aromatic molecule.

If we were to assume that $\mathrm{CO}$ in our $(2 \times 2)$ structure sits on 3 -fold hollow sites, then the STM images imply that the center of the triangular shape is either on a top site or on a 3-fold hollow site of the same symmetry (i.e., fcc or hcp site). The latter assignment would be in line with the LEED experiments [20] that found that CO indeed sits on 3-fold hollow sites of hcp symmetry when coadsorbed with benzene in either a 1:1 or 1:2 ratio. If xylene behaves similarly to benzene, we could expect that it might induce the surrounding $\mathrm{CO}$ to also adopt hcp binding sites. The STM results would then imply that the site change from top to hollow is propagated well beyond the nearest neighbor positions of the xylene molecule, an interesting result that might have important implications in the chemical and catalytic properties of the substrate. Of course, there is no firm basis yet to justify this model, but the issue is interesting enough to call for additional experiments.

Atomically resolved images of the bare $\mathrm{Rh}$ substrate obtained simultaneously with those of areas covered with adsorbed molecules would go a long way toward determining the adsorption sites. Unfortunately, we were not able to obtain such images. Another possibility is to resort to theoretical simulations of the STM images. For example, the images of the unit cell of the CO structure show a marked asymmetry (bottom image, 
Figure 8-10). This image shows that the tunneling probability in the center of one half of the cell is larger than in the other half. The unit cell is made of two non-equivalent equilateral triangles sharing a side. For the top site $(2 \times 2) \mathrm{CO}$ structure, each half-unit cell triangle is centered on a 3-fold hollow site, differing only in their second-layer $\mathrm{Rh}$ atom positions. For the model in which $\mathrm{CO}$ sits on 3-fold hollow sites, however, the two halfunit cell triangles differ in their Rh positions in the first layer, with one triangle containing a $\mathrm{Rh}$ atom at its center and the other containing a 3 -fold hollow site. We expect the difference of tunnel probabilities in the latter case to be larger than in the former, thus explaining the STM observation. Since theoretical simulations have not yet been performed, it is not possible to make any definite conclusions at this point.

Because of the clear differences in their appearance (diamond shapes for paraxylene and triangular shapes for meta-xylene molecules), we can easily differentiate between these molecules in coadsorption experiments. The perfect intermixing of the two isomers shows that the interaction energy between them is similar to that between paraxylene or meta-xylene molecules when adsorbed alone on that metal surface. It also indicates that the mixing heat of the two isomers is small and that entropy is the driving force for mixing.

The fact that the two xylene isomers have similar corrugations $(0.6 \pm 0.1 \AA)$ implies that they interact with the $\mathrm{Rh}(111)$ surface in a similar manner. If the molecules were bound to the surface through the methyl groups, meta-xylene could orient its aromatic ring at an angle to the surface, since both methyl groups could still be in contact with the surface. Para-xylene, on the other hand, would have to lie with its aromatic ring parallel to the surface, so that both methyl groups could be close to the surface. This 
behavior has been observed for xylene isomers on the $\mathrm{Pt}(111)$ surface at $370 \mathrm{~K}$ [17]. However, since the corrugations of the two isomers are similar, there is probably no tilting of the meta-xylene at $300 \mathrm{~K}$ and the dominant bonding interaction is through the $\pi$ orbitals in the aromatic ring. 


\section{References}

[1] M.J. Allen, M. Balooch, S. Subbiah, R.J. Tench, R. Balhorn, W. Siekhaus, Ultramicroscopy 42-44, pt. B (1992) 1049.

[2] T.A. Jung, R.R. Schlittler, J.K. Gimzewski, Nature 386 (1997) 696.

[3] J.-T. Kim, T. Kawai, J. Yoshinobu, M. Kawai, Surf. Sci. 360 (1996) 50.

[4] M. Kasaya, H. Tabata, T. Kawai, Surf. Sci. 357-358 (1996) 195.

[5] H. Tanaka, T. Kawai, Japanese J. Appl. Phys. 35 (1996) 3759.

[6] B.G. Frederick, Q. Chen, S.M. Barlow, N.G. Condon, F.M. Leibsle, N.V. Richardson, Surf. Sci. 352-354 (1996) 238.

[7] E.R. Frank, X.X. Chen, R.J. Hamers, Surf. Sci. 334 (1995) L709.

[8] M. Kanai, T. Kawai, K. Motai, X.D. Wang, T. Hashizume, T. Sakura, Surf. Sci. 329 (1995) L619.

[9] A.J. Fisher, P.E. Blochl, Phys. Rev. Lett. 70 (1993) 3263.

[10] S. Chiang, V.M. Hallmark, K.-P. Meinhardt, K. Hafner, J. Vac. Sci. Technol. B 12 (1994) 1957.

[11] P. Sautet, M.-L. Bocquet, Surf. Sci. 304 (1994) L445.

[12] D.N. Futaba, S. Chiang, J. Vac. Sci. Technol. A 15 (1997) 1295.

[13] H.A. Yoon, M. Salmeron, G.A. Somorjai, Surf. Sci. 373 (1997) 300.

[14] H.A. Yoon, M. Salmeron, G.A. Somorjai, Surf. Sci. 395 (1998) 268.

[15] H.A. Yoon, Ph.D. Thesis, University of California, Berkeley, 1996.

[16] D.M. Zeglinski, D.F. Ogletree, T.P. Beebe, R.Q. Hwang, G.A. Somorjai, M. Salmeron, Rev. Sci. Instrum. 61 (1990) 3769. 
[17] V.M. Hallmark, S. Chiang, K.-P. Meinhardt, K. Hafner, Phys. Rev. Lett. 70 (1993) 3740

[18] D. Futaba, S. Chiang, personal communication.

[19] A. Beutler, E. Lundgren, R. Nyholm, J.N. Andersen, B. Selik, D. Heskett, Surf. Sci. 371 (1997) 381.

[20] P.A. Thiel, J.T. Yeats, Jr., W.H. Weinberg, Surf. Sci. 82 (1979) 59.

[21] D.H. Wei, D.C. Skelton, S.D. Kevan, Surf. Sci. 381 (1997) 49.

[22] A. Barbieri, M.A. Van Hove, G.A. Somorjai, Surf. Sci. 306 (1994) 261. 


\section{Chapter 9: Conclusion}

Since the introduction of the Scanning Tunneling Microscope in the early 1980's the work of understanding surface structure and chemistry has matured. With the advent of an instrument which can give a topographic image of the surface with Angstrom resolution, studies of the atomic and molecular properties of surfaces have become a significant part of surface science. However, since the technique is still relatively new only second or third generation microscopes are being used - there are still many areas of investigation which are open. The study of adsorbed layers on the surface, which has been a focus of LEED crystallography, can be supplemented with STM experiments that show the disordered areas that are not evident from the LEED data.

In this work, the focus of experiments has been on the ordered overlayers on the (111) surface of rhodium. These adsorbate and coadsorbate systems include molecules with are interesting because of their industrial and catalytic reaction ( $\mathrm{CO}$, cyclohexane), the induced ordering of systems by coadsorption ( $\mathrm{CO}$ plus ethylidyne), and systems where 
the molecules have interesting properties which may affect their STM images (para-and meta-xylene).

In the case of $\mathrm{CO}$, the structure of the low coverage $(2 \times 2)$ overlayer has been ovbserved with the STM. The symmetry of the unit cell in this layer suggests that the CO is adsorbed in the 3 -fold hollow sites. There were also two higher coverage structures images that do not correspond to any structures previously reported in the literature. These are two domains with $(\sqrt{ } 7 x \sqrt{7})$ unit cells. One of these is composed of trimers of $\mathrm{CO}$ and has three $\mathrm{CO}$ molecules in each unit cell. The other structure has an additional $\mathrm{CO}$ molecule, making a total of four. This extra $\mathrm{CO}$ sits on a top site, which explains the increased tunneling current indicated by a bright feature in the unit cell.

When $\mathrm{CO}$ is added to a disordered ethylidyne covered surface, an ordered overlayer forms with $\mathrm{c}(4 \times 2)$ symmetry. This was observed by LEED as well as with the STM. In the STM images, ordered domains were observed, sometimes two domains in the same image. The ordered domains only contain one type of feature, not two as would be expected (one for $\mathrm{CO}$ and one for ethylidyne). This feature has a larger corrugation than has been observed for $\mathrm{CO}$ on the surface. Due to the difference in heights of the molecules when they are adsorbed on the surface, as well as energetic considerations, it is most likely that the imaged molecule is ethylidyane, not $\mathrm{CO}$.

Three cyclic molecules were adsorbed on the surface, cyclohexane, cyclohexene and 1,4-cyclohexadiene, in order to examine whether these molecules dehydrogenated spontaneously on the surface. No ordered domains were observed for cyclohexane. Cyclohexene adsorbed on the surface in two different ordered structures, both of which correspond to the structures formed by the coadsorption of benzene and $\mathrm{CO}$. The 
molecules is both of these domains, the $(3 \times 3)$ and the $c(2 \sqrt{3} \times 4)$ structures, had high symmetry. Based on these two considerations it is most likely that the cyclohexane has dehydrogenated to form benzene. When 1,4-cyclohexadiene is adsorbed on the surface, it also forms the $c(2 \sqrt{3} \times 4)$ structure, indicating the conversion to benzene. However, when the surface is exposed to hydrogen gas before the adsorption of 1,4 cyclohexadiene, then two different ordered domains are observed on the surface. In this case the domain with the larger unit cell is likely the $c(2 \sqrt{ } 3 \times 4)$ structure. The domain with the smaller unit cell may be unconverted 1,4 cyclohexadiene caused by the blockage of the $H$ atom adsorption sites by the exposure to hydrogen gas. More experiments would be needed to confirm this suspicion.

Two aromatic molecules of the benzene family, para- and meta-xylene, were imaged with STM on $\mathrm{Rh}(111)$ at room temperature. The molecular images have peculiar shapes that reflect their atomic and orbital structures. These shapes are diamonds and triangles with two bright corners, respectively. These have been assigned to the methyl groups based on symmetry and on steric considerations. Para-xylene forms ordered domains with three orientations, with one mirror plane of the molecule aligned parallel to the [112]-type directions. Meta-xylene molecules do not form ordered domains at room temperature, although individual molecules have a well-defined angular orientation relative to the substrate. Molecular motion in the form of rotations and small displacements occurs for both molecules at domain boundaries or in disordered areas where the packing density is smaller. In the diluted case of low coverage, the molecules diffuse readily and can not be completely imaged by the tip. Only at the step edges is the mobility low enough (due to stronger binding) for stable imaging. 
Coadsorption with $\mathrm{CO}$ was investigated in the case of meta-xylene. $\mathrm{CO}$ prevented diffusion of the molecules and formed a (2x2) structure surrounding the isolated xylene molecules. From the STM images, we conclude that the center of the triangular metaxylene image is located in either the bridge or 3-fold hollow sites if the CO molecule adsorbs on top sites, and on top or 3-fold hollow sites if the CO molecule adsorbs on a 3fold hollow site. 